TITLE:

\title{
Variations of Mixed Hodge Structure and Semipositivity Theorems
}

\author{
$\operatorname{AUTHOR}(\mathrm{S})$ : \\ Fujino, Osamu; Fujisawa, Taro
}

\section{CITATION:}

Fujino, Osamu ... [et al]. Variations of Mixed Hodge Structure and Semipositivity Theorems. Publications of the Research Institute for Mathematical Sciences 2014, 50(4): 589-661

\section{ISSUE DATE:}

2014

URL:

http://hdl.handle.net/2433/200189

\section{RIGHT:}

(C) 2014 Research Institute for Mathematical Sciences, Kyoto University.; This is not the published version. Please cite only the published version.; この論文は出版社版でありません。引用の際には出版社版を ご確認ご利用ください。 


\title{
Variations of mixed Hodge structure and semipositivity theorems
}

by

\author{
Osamu Fujino, Taro Fujisawa
}

\begin{abstract}
We discuss the variations of mixed Hodge structure for cohomology with compact support of quasi-projective simple normal crossing pairs. We show that they are graded polarizable admissible variations of mixed Hodge structure. Then we prove a generalization of the Fujita-Kawamata semipositivity theorem.

2010 Mathematics Subject Classification: Primary 14D07; Secondary 14C30, 14E30, 32G20. Keywords: variations of mixed Hodge structure, cohomology with compact support, canonical extensions of Hodge bundles, semipositivity theorems.
\end{abstract}

\section{Contents}

$\S 1$ Introduction 1

$\S 2$. Preliminaries 7

$\S 3$. Generalities on variation of mixed Hodge structure 9

$\S 4$. Variation of mixed Hodge structure of geometric origin 17

$\S 5$. Semipositivity theorem 41

$\S 6$. Vanishing and torsion-free theorems 51

$\S 7$. Higher direct images of $\log$ canonical divisors $\quad 55$

$\S 8$. Examples 67

References 73

Communicated by S. Mukai. Received September 24, 2012. Revised April 23, 2013, December 6, 2013, March 17, 2014.

O. Fujino: Department of Mathematics, Graduate School of Science, Kyoto University, Kyoto 606-8502, Japan;

e-mail: fujino@math.kyoto-u.ac.jp

T. Fujisawa: Tokyo Denki University, School of Engineering, Department of Mathematics, Tokyo, Japan;

e-mail: fujisawa@mail.dendai.ac.jp

(C) 201x Research Institute for Mathematical Sciences, Kyoto University. All rights reserved. 


\section{$\S 1$. Introduction}

Let $X$ be a simple normal crossing divisor on a smooth projective variety $M$ and let $B$ be a simple normal crossing divisor on $M$ such that $X+B$ is simple normal crossing on $M$ and that $X$ and $B$ have no common irreducible components. Then the pair $(X, D)$, where $D=\left.B\right|_{X}$, is a typical example of simple normal crossing pairs. In this situation, a stratum of $(X, D)$ is an irreducible component of $T_{i_{1}} \cap \cdots \cap T_{i_{k}} \subset X$ for some $\left\{i_{1}, \cdots, i_{k}\right\} \subset I$, where $X+B=\sum_{i \in I} T_{i}$ is the irreducible decomposition of $X+B$. For the precise definition of simple normal crossing pairs, see Definition 2.1 below. We note that simple normal crossing pairs frequently appear in the study of the log minimal model program for higher dimensional algebraic varieties with bad singularities. The first author has already investigated the mixed Hodge structures for $H_{c}^{\bullet}(X \backslash D, \mathbb{Q})$ in $[\mathrm{F} 7$, Chapter 2] to obtain various vanishing theorems (see also [F17]). In this paper, we show that their variations are graded polarizable admissible variations of mixed Hodge structure. Then we prove a generalization of the Fujita-Kawamata semipositivity theorem. Our formulation of the Fujita-Kawamata semipositivity theorem is different from Kawamata's original one. However, it is more suited for our studies of simple normal crossing pairs.

The following theorem is a corollary of Theorem 7.1 and Theorem 7.3, which are our main results of this paper (cf. [Kw1, Theorem 5], [Ko2, Theorem 2.6], [N1, Theorem 1], [F4, Theorems 3.4 and 3.9], [Kw3, Theorem 1.1], and so on).

Theorem 1.1 (Semipositivity theorem (cf. Theorem 7.1 and Theorem 7.3)). Let $(X, D)$ be a simple normal crossing pair such that $D$ is reduced and let $f: X \longrightarrow Y$ be a projective surjective morphism onto a smooth complete algebraic variety $Y$. Assume that every stratum of $(X, D)$ is dominant onto $Y$. Let $\Sigma$ be a simple normal crossing divisor on $Y$ such that every stratum of $(X, D)$ is smooth over $Y^{*}=Y \backslash \Sigma$. Then $R^{p} f_{*} \omega_{X / Y}(D)$ is locally free for every $p$. We put $X^{*}=f^{-1}\left(Y^{*}\right)$, $D^{*}=\left.D\right|_{X^{*}}$, and $d=\operatorname{dim} X-\operatorname{dim} Y$. We further assume that all the local mon-

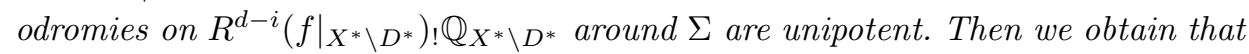
$R^{i} f_{*} \omega_{X / Y}(D)$ is a semipositive locally free sheaf on $Y$.

We note the following definition.

Definition 1.2 (Semipositivity in the sense of Fujita-Kawamata). A locally free sheaf $\mathcal{E}$ of finite rank on a complete algebraic variety $X$ is said to be semipositive (in the sense of Fujita-Kawamata) if and only if $\mathcal{O}_{\mathbb{P}_{X}(\mathcal{E})}(1)$ is nef on $\mathbb{P}_{X}(\mathcal{E})$.

In [Kw3], Kawamata obtained a weaker result similar to Theorem 1.1 (see [Kw3, Theorem 1.1]). It is not surprising because both [Kw3] and this paper grew 
from the same question raised by Valery Alexeev and Christopher Hacon. For details on Kawamata's approach, we recommend the reader to see [FFS], where we use the theory of mixed Hodge modules to give an alternative proof of Theorem 1.1. Note that $[$ FFS] was written after this paper was circulated.

The semipositivity of $R^{i} f_{*} \omega_{X / Y}(D)$ in Theorem 1.1 follows from a purely Hodge theoretic semipositivity theorem: Theorem 5.21. In the proof of Theorem 1.1, we use the semipositivity of $\left(\mathrm{Gr}_{F}^{a} \mathcal{V}\right)^{*}$ in Theorem 1.3, where $\left(\mathrm{Gr}_{F}^{a} \mathcal{V}\right)^{*}=$ $\mathcal{H o m}_{\mathcal{O}_{Y}}\left(\operatorname{Gr}_{F}^{a} \mathcal{V}, \mathcal{O}_{Y}\right)$. We do not need the semipositivity of $F^{b} \mathcal{V}$ in Theorem 1.3 for Theorem 1.1. For more details, see the discussion in 1.6 below.

Theorem 1.3 (Hodge theoretic semipositivity theorem (cf. Theorem 5.21)). Let $X$ be a smooth complete complex algebraic variety, $D$ a simple normal crossing divisor on $X, \mathcal{V}$ a locally free $\mathcal{O}_{X}$-module of finite rank equipped with a finite increasing filtration $W$ and a finite decreasing filtration $F$. We assume the following:

(1) $F^{a} \mathcal{V}=\mathcal{V}$ and $F^{b+1} \mathcal{V}=0$ for some $a<b$

(2) $\operatorname{Gr}_{F}^{p} \operatorname{Gr}_{m}^{W} \mathcal{V}$ is a locally free $\mathcal{O}_{X}$-module of finite rank for all $m, p$.

(3) For all $m, \mathrm{Gr}_{m}^{W} \mathcal{V}$ admits an integrable logarithmic connection $\nabla_{m}$ with the nilpotent residue morphisms which satisfies the conditions

$$
\nabla_{m}\left(F^{p} \operatorname{Gr}_{m}^{W} \mathcal{V}\right) \subset \Omega_{X}^{1}(\log D) \otimes F^{p-1} \operatorname{Gr}_{m}^{W} \mathcal{V}
$$

for all $p$.

(4) The triple $\left.\left(\operatorname{Gr}_{m}^{W} \mathcal{V}, F \mathrm{Gr}_{m}^{W} \mathcal{V}, \nabla_{m}\right)\right|_{X \backslash D}$ underlies a polarizable variation of $\mathbb{R}$ Hodge structure of weight $m$ for every integer $m$.

Then $\left(\operatorname{Gr}_{F}^{a} \mathcal{V}\right)^{*}$ and $F^{b} \mathcal{V}$ are semipositive.

In this paper, we concentrate on the Hodge theoretic aspect of the FujitaKawamata semipositivity theorem (cf. [Z], [Kw1], [Ko2], [N1], [F4], and [FFS]). On the other hand, there are many results related to the Fujita-Kawamata semipositivity theorem from the analytic viewpoint (cf. $[\mathrm{Ft}],[\mathrm{Be}],[\mathrm{BeP}],[\mathrm{MT}]$, and so on). Note that Griffiths's pioneering work on the variation of Hodge structure (cf. [G]) is a starting point of the Fujita-Kawamata semipositivity theorem. For a related topic, see [Moc]. Mochizuki's approach is completely different from ours and has more arithmetic geometrical flavors.

As a special case of Theorem 1.1, we obtain the following theorem: Theorem 7.7 .

Theorem 1.4 (cf. [Kw1, Theorem 5], [Ko2, Theorem 2.6], and [N1, Theorem 1]). Let $f: X \longrightarrow Y$ be a projective morphism between smooth complete algebraic varieties which satisfies the following conditions: 
(i) There is a Zariski open subset $Y^{*}$ of $Y$ such that $\Sigma=Y \backslash Y^{*}$ is a simple normal crossing divisor on $Y$.

(ii) We put $X^{*}=f^{-1}\left(Y^{*}\right)$. Then $\left.f\right|_{X^{*}}$ is smooth.

(iii) The local monodromies of $R^{d+i}\left(\left.f\right|_{X^{*}}\right)_{*} \mathbb{C}_{X^{*}}$ around $\Sigma$ are unipotent, where $d=\operatorname{dim} X-\operatorname{dim} Y$.

Then $R^{i} f_{*} \omega_{X / Y}$ is a semipositive locally free sheaf on $Y$.

We note that Theorem 1.4 was first proved by Kawamata (cf. [Kw1, Theorem 5]) under the extra assumptions that $i=0$ and that $f$ has connected fibers. The above statement follows from [Ko2, Theorem 2.6] or [N1, Theorem 1] (see also [F5, Theorem 5.4]). We also note that, by Poincaré-Verdier duality, $R^{d+i}\left(\left.f\right|_{X^{*}}\right)_{*} \mathbb{C}_{X^{*}}$ is the dual local system of $R^{d-i}\left(\left.f\right|_{X^{*}}\right)_{*} \mathbb{C}_{X^{*}}$ in Theorem 1.4. In [Ko2] and [N1], the variation of Hodge structure on $R^{d+i}\left(\left.f\right|_{X^{*}}\right)_{*} \mathbb{C}_{X^{*}}$ is investigated for the proof of Theorem 1.4. On the other hand, in this paper, we concentrate on the variation of Hodge structure on $R^{d-i}\left(\left.f\right|_{X^{*}}\right)_{*} \mathbb{C}_{X^{*}}$ for Theorem 1.4.

The following example shows that the assumption (2) in Theorem 1.3 is indispensable. For related examples, see [SZ, (3.15) and (3.16)]. In the proof of Theorem 1.1 , the admissibility of the graded polarizable variation of $\mathbb{Q}$-mixed Hodge structure on $R^{d-i}\left(\left.f\right|_{X^{*} \backslash D^{*}}\right) ! \mathbb{Q}_{X^{*} \backslash D^{*}}$, which is proved in Theorem 4.15, assures us the existence of the extension of the Hodge filtration satisfying the assumption (2). Note that the notion of admissibility is due to Steenbrink-Zucker [SZ] and Kashiwara $[\mathrm{Ks}]$.

Example 1.5. Let $\mathbb{V}$ be a 2 -dimensional $\mathbb{Q}$-vector space with basis $\left\{e_{1}, e_{2}\right\}$. We give an increasing filtration $W$ on $\mathbb{V}$ by $W_{-1} \mathbb{V}=0, W_{0} \mathbb{V}=W_{1} \mathbb{V}=\mathbb{Q} e_{1}$, and $W_{2} \mathbb{V}=\mathbb{V}$. The constant sheaf on $\mathbb{P}^{1}$ whose fibers are $\mathbb{V}$ is denoted by the same letter $\mathbb{V}$, on which an increasing filtration $W$ is given as above. We consider $\mathcal{V}=$ $\mathcal{O}_{\mathbb{P}^{1}} \otimes \mathbb{V}=\mathcal{O} e_{1} \oplus \mathcal{O} e_{2}$ on $\mathbb{P}^{1}$. We set a decreasing filtration $F$ on $\left.\mathcal{V}\right|_{\mathbb{C}^{*}}$ by

$$
F^{0}\left(\left.\mathcal{V}\right|_{\mathbb{C}^{*}}\right)=\left.\mathcal{V}\right|_{\mathbb{C}^{*}}, F^{1}\left(\left.\mathcal{V}\right|_{\mathbb{C}^{*}}\right)=\mathcal{O}_{\mathbb{C}^{*}}\left(t^{-1} e_{1}+e_{2}\right), F^{2}\left(\left.\mathcal{V}\right|_{\mathbb{C}^{*}}\right)=0,
$$

where $t$ is the coordinate function of $\mathbb{C} \subset \mathbb{P}^{1}$. We can easily check that

$$
\left(\left.(\mathbb{V}, W)\right|_{\mathbb{C}^{*}},\left(\left.\mathcal{V}\right|_{\mathbb{C}^{*}}, F\right)\right)
$$

is a graded polarizable variation of $\mathbb{Q}$-mixed Hodge structure on $\mathbb{C}^{*}$. In this case, we can not extend the Hodge filtration $F$ on $\left.\mathcal{V}\right|_{\mathbb{C}^{*}}$ to the filtration $F$ on $\mathcal{V}$ satisfying the assumption (2) in Theorem 1.3. In particular, the above variation of $\mathbb{Q}$-mixed Hodge structure is not admissible.

We note that we can extend the Hodge filtration $F$ on $\left.\mathcal{V}\right|_{\mathbb{C}^{*}}$ to the filtration $F$ on $\mathcal{V}$ such that $F^{2} \mathcal{V}=0, F^{1} \mathcal{V} \simeq \mathcal{O}_{\mathbb{P}^{1}}(-1)$, and $F^{0} \mathcal{V}=\mathcal{V}$ with $\operatorname{Gr}_{F}^{0} \mathcal{V} \simeq$ 
$\mathcal{O}_{\mathbb{P}^{1}}(1)$. In this case, $F^{1} \mathcal{V}$ and $\left(\mathrm{Gr}_{F}^{0} \mathcal{V}\right)^{*}$ are not semipositive. This means that a naive generalization of the Fujita-Kawamata semipositivity theorem for graded polarizable variations of $\mathbb{Q}$-mixed Hodge structure is false.

As an application of Theorem 1.1, the first author proves a semipositivity theorem for families of singular varieties in [F16]. It is a generalization of [Ko4, 4.12. Theorem] and implies that the moduli functor of stable varieties is semipositive in the sense of Kollár (see [Ko4, 2.4. Definition]). Therefore, it will play crucial roles for the projectivity of the moduli spaces of higher-dimensional stable varieties. For details, see [Ko4], [F15], and [F16].

We give a sketch of the proof of Theorem 1.1 for the reader's convenience.

1.6 (Sketch of the proof of Theorem 1.1). In Theorem 1.1, we see that the local system $R^{d-i}\left(\left.f\right|_{\left.X^{*} \backslash D^{*}\right) ! \mathbb{Q}_{X^{*}} \backslash D^{*}}\right.$ underlies an admissible variation of $\mathbb{Q}$-mixed Hodge structure by Theorem 4.15. Let $\mathcal{V}$ be the canonical extension of the locally free sheaf $\left(R^{d-i}\left(\left.f\right|_{X^{*} \backslash D^{*}}\right) ! \mathbb{Q}_{X^{*} \backslash D^{*}}\right) \otimes \mathcal{O}_{Y^{*}}$. Then we can prove $R^{d-i} f_{*} \mathcal{O}_{X}(-D) \simeq$ $\operatorname{Gr}_{F}^{0} \mathcal{V}$ where $F$ is the canonical extension of the Hodge filtration. Note that the admissibility ensures the existence of the canonical extensions of the Hodge bundles (cf. Proposition 3.12 and Remark 7.4). We also note that we use an explicit description of the canonical extension of the Hodge filtration in order to prove $R^{d-i} f_{*} \mathcal{O}_{X}(-D) \simeq \operatorname{Gr}_{F}^{0} \mathcal{V}$ when $Y$ is a curve. By Grothendieck duality, we obtain $R^{i} f_{*} \omega_{X / Y}(D) \simeq\left(\operatorname{Gr}_{F}^{0} \mathcal{V}\right)^{*}$. Therefore, $R^{i} f_{*} \omega_{X / Y}(D)$ is semipositive by Theorem 1.3. It is very important to note that the local system $R^{d-i}\left(\left.f\right|_{\left.X^{*} \backslash D^{*}\right) ! \mathbb{Q}_{X^{*}} \backslash D^{*}}\right.$ is not necessarily the dual local system of $R^{d+i}\left(\left.f\right|_{X^{*} \backslash D^{*}}\right)_{*} \mathbb{Q}_{X^{*} \backslash D^{*}}$ because $X$ is not a smooth variety but a simple normal crossing variety. In the proof of Theorem 1.1, we use the recent developments of the theory of partial resolution of singularities for reducible varieties (see $[\mathrm{BiM}]$ and $[\mathrm{BiP}]$ ) for the reduction to simpler cases.

We quickly explain the reason why we use mixed Hodge structures for cohomology with compact support.

1.7 (Mixed Hodge structure for cohomology with compact support). Let $X$ be a smooth projective variety and let $D$ be a simple normal crossing divisor on $X$. After Iitaka introduced the notion of logarithmic Kodaira dimension, $\mathcal{O}_{X}\left(K_{X}+D\right)$ plays important roles in the birational geometry, where $K_{X}$ is the canonical divisor of $X$. In the traditional birational geometry, $\mathcal{O}_{X}\left(K_{X}+D\right)$ is recognized to be $\Omega_{X}^{\operatorname{dim} X}(\log D)$. Therefore, the Hodge to de Rham spectral sequence

$$
E_{1}^{p, q}=H^{q}\left(X, \Omega_{X}^{p}(\log D)\right) \Longrightarrow H^{p+q}(X \backslash D, \mathbb{C})
$$


arising from the mixed Hodge structures on $H^{\bullet}(X \backslash D, \mathbb{C})$ is useful. The first author recognizes $\mathcal{O}_{X}\left(K_{X}+D\right)$ as

$$
\mathcal{H o m}_{\mathcal{O}_{X}}\left(\mathcal{O}_{X}(-D), \mathcal{O}_{X}\left(K_{X}\right)\right)
$$

or

$$
R \mathcal{H o m}_{\mathcal{O}_{X}}\left(\mathcal{O}_{X}(-D), \omega_{X}^{\bullet}\right)[-\operatorname{dim} X]
$$

where $\omega_{X}^{\bullet}=\mathcal{O}_{X}\left(K_{X}\right)[\operatorname{dim} X]$ is the dualizing complex of $X$. Furthermore, $\mathcal{O}_{X}(-D)$ can be interpreted as the 0 -th term of the complex

$$
\Omega_{X}^{\bullet}(\log D) \otimes \mathcal{O}_{X}(-D) .
$$

By this observation, we can use the following Hodge to de Rham spectral sequence

$$
E_{1}^{p, q}=H^{q}\left(X, \Omega_{X}^{p}(\log D) \otimes \mathcal{O}_{X}(-D)\right) \Longrightarrow H_{c}^{p+q}(X \backslash D, \mathbb{C})
$$

arising from the mixed Hodge structures on cohomology groups $H_{c}^{\bullet}(X \backslash D, \mathbb{C})$ of $X \backslash D$ with compact support and obtain various powerful vanishing theorems. For details and many applications, see [F7, Chapter 2], [F8], [F10], [F11, Section 5], [F12], [F15], and [F17]. Therefore, it is very natural to consider the variations of such mixed Hodge structures.

We summarize the contents of this paper. Section 2 is a preliminary section. Section 3 collects some generalities on variations of mixed Hodge structure. In Section 4, we discuss the variations of mixed Hodge structure for simple normal crossing pairs. We show that they are graded polarizable and admissible. Theorem 4.15 is the main result of Section 4. In Section 5, we discuss a purely Hodge theoretic aspect of the Fujita-Kawamata semipositivity theorem. Our formulation is different from Kawamata's original one but is suited for our results in Section 7. In Section 6, we discuss some generalizations of vanishing and torsion-free theorems for quasi-projective simple normal crossing pairs. They are necessary for the arguments in Section 7. Section 7 is the main part of this paper. Here, we characterize higher direct images of log canonical divisors by using canonical extensions of Hodge bundles (cf. Theorem 7.1 and Theorem 7.3). It is a generalization of the results by Yujiro Kawamata, Noboru Nakayama, János Kollár, Morihiko Saito, and Osamu Fujino. In Section 8, we treat some examples, which help us understand the Fujita-Kawamata semipositivity theorem, Viehweg's weak positivity theorem, and so on, in details.

Let us recall basic definitions and notation.

Notation. For a proper morphism $f: X \longrightarrow Y$, the exceptional locus, which is denoted by $\operatorname{Exc}(f)$, is the locus where $f$ is not an isomorphism. $\mathbb{R}$ (resp. $\mathbb{Q}$ ) denotes the set of real (resp. rational) numbers. $\mathbb{Z}$ denotes the set of integers. 
1.8 (Divisors, $\mathbb{Q}$-divisors, and $\mathbb{R}$-divisors). For an $\mathbb{R}$-Weil divisor $D=\sum_{j=1}^{r} d_{j} D_{j}$ such that $D_{i}$ is a prime divisor for every $i$ and that $D_{i} \neq D_{j}$ for $i \neq j$, we define the round-up $\lceil D\rceil=\sum_{j=1}^{r}\left\lceil d_{j}\right\rceil D_{j}$ (resp. the round-down $\lfloor D\rfloor=\sum_{j=1}^{r}\left\lfloor d_{j}\right\rfloor D_{j}$ ), where for any real number $x,\lceil x\rceil$ (resp. $\lfloor x\rfloor$ ) is the integer defined by $x \leq\lceil x\rceil<x+1$ (resp. $x-1<\lfloor x\rfloor \leq x$ ). The fractional part $\{D\}$ of $D$ denotes $D-\lfloor D\rfloor$. We call $D$ a boundary (resp. subboundary) $\mathbb{R}$-divisor if $0 \leq d_{j} \leq 1$ (resp. $d_{j} \leq 1$ ) for every $j$. $\mathbb{Q}$ linear equivalence (resp. $\mathbb{R}$-linear equivalence) of two $\mathbb{Q}$-divisors (resp. $\mathbb{R}$-divisors) $B_{1}$ and $B_{2}$ is denoted by $B_{1} \sim_{\mathbb{Q}} B_{2}\left(\right.$ resp. $\left.B_{1} \sim_{\mathbb{R}} B_{2}\right)$.

1.9 (Singularities of pairs). Let $X$ be a normal variety and let $\Delta$ be an effective $\mathbb{R}$-divisor on $X$ such that $K_{X}+\Delta$ is $\mathbb{R}$-Cartier. Let $f: X \longrightarrow Y$ be a resolution such that $\operatorname{Exc}(f) \cup f_{*}^{-1} \Delta$ has a simple normal crossing support, where $f_{*}^{-1} \Delta$ is the strict transform of $\Delta$ on $Y$. We can write

$$
K_{Y}=f^{*}\left(K_{X}+\Delta\right)+\sum_{i} a_{i} E_{i} .
$$

We say that $(X, \Delta)$ is $\log$ canonical (lc, for short) if $a_{i} \geq-1$ for every $i$. We usually write $a_{i}=a\left(E_{i}, X, \Delta\right)$ and call it the discrepancy coefficient of $E$ with respect to $(X, \Delta)$.

If $(X, \Delta)$ is $\log$ canonical and there exist a resolution $f: Y \longrightarrow X$ and a divisor $E$ on $Y$ such that $a(E, X, \Delta)=-1$, then $f(E)$ is called a $\log$ canonical center (lc center, for short) with respect to $(X, \Delta)$.

It is very important to understand the following example.

1.10 (A basic example). Let $X$ be a smooth variety and let $\Delta$ be a reduced simple normal crossing divisor on $X$. Then the pair $(X, \Delta)$ is $\log$ canonical. Let $\Delta=$ $\sum_{i \in I} \Delta_{i}$ be the irreducible decomposition of $\Delta$. Then a subvariety $W$ of $X$ is a $\log$ canonical center with respect to $(X, \Delta)$ if and only if $W$ is an irreducible component of $\Delta_{i_{1}} \cap \cdots \cap \Delta_{i_{k}}$ for some $\left\{i_{1}, \cdots, i_{k}\right\} \subset I$.

We will work over $\mathbb{C}$, the field of complex numbers, throughout this paper.

\section{§2. Preliminaries}

Let us recall the definition of simple normal crossing pairs.

Definition 2.1 (Simple normal crossing pairs). We say that the pair $(X, D)$ is simple normal crossing at a point $a \in X$ if $X$ has a Zariski open neighborhood $U$ of $a$ that can be embedded in a smooth variety $Y$, where $Y$ has regular system of parameters $\left(x_{1}, \cdots, x_{p}, y_{1}, \cdots, y_{r}\right)$ at $a=0$ in which $U$ is defined by a monomial 
equation

$$
x_{1} \cdots x_{p}=0
$$

and

$$
D=\left.\sum_{i=1}^{r} \alpha_{i}\left(y_{i}=0\right)\right|_{U}, \quad \alpha_{i} \in \mathbb{R} .
$$

We say that $(X, D)$ is a simple normal crossing pair if it is simple normal crossing at every point of $X$. When $D$ is the zero divisor for a simple normal crossing pair $(X, D), X$ is called a simple normal crossing variety. If $(X, D)$ is a simple normal crossing pair, then $X$ has only Gorenstein singularities. Thus, it has an invertible dualizing sheaf $\omega_{X}$. Therefore, we can define the canonical divisor $K_{X}$ such that $\omega_{X} \simeq \mathcal{O}_{X}\left(K_{X}\right)$. It is a Cartier divisor on $X$ and is well-defined up to linear equivalence.

We say that a simple normal crossing pair $(X, D)$ is embedded if there exists a closed embedding $\iota: X \hookrightarrow M$, where $M$ is a smooth variety of dimension $\operatorname{dim} X+1$.

Let $X$ be a simple normal crossing variety and let $D$ be a Cartier divisor on $X$. If $(X, D)$ is a simple normal crossing pair and $D$ is reduced, then $D$ is called a simple normal crossing divisor on $X$.

We note that a simple normal crossing pair is called a semi-snc pair in [Ko6, Definition 1.10].

Definition 2.2 (Strata and permissibility). Let $X$ be a simple normal crossing variety and let $X=\bigcup_{i \in I} X_{i}$ be the irreducible decomposition of $X$. A stratum of $X$ is an irreducible component of $X_{i_{1}} \cap \cdots \cap X_{i_{k}}$ for some $\left\{i_{1}, \cdots, i_{k}\right\} \subset I$. A Cartier divisor $B$ on $X$ is permissible if $B$ contains no strata of $X$ in its support. A finite $\mathbb{Q}$-linear (resp. $\mathbb{R}$-linear) combination of permissible Cartier divisors is called a permissible $\mathbb{Q}$-divisor (resp. $\mathbb{R}$-divisor) on $X$.

Let $(X, D)$ be a simple normal crossing pair such that $D$ is a boundary $\mathbb{R}$ divisor on $X$. Let $\nu: X^{\nu} \longrightarrow X$ be the normalization. We define $\Theta$ by the formula

$$
K_{X^{\nu}}+\Theta=\nu^{*}\left(K_{X}+D\right) .
$$

Then a stratum of $(X, D)$ is an irreducible component of $X$ or the $\nu$-image of a log canonical center of $\left(X^{\nu}, \Theta\right)$. We note that $\left(X^{\nu}, \Theta\right)$ is $\log$ canonical (cf. 1.10). When $D=0$, this definition is compatible with the aforementioned case. An $\mathbb{R}$-Cartier $\mathbb{R}$-divisor $B$ on $X$ is permissible with respect to $(X, D)$ if $B$ contains no strata of $(X, D)$ in its support. If $B$ is a permissible $\mathbb{R}$-Cartier $\mathbb{R}$-divisor with respect to $(X, D)$, then we can easily check that

$$
B=\sum_{i} b_{i} B_{i}
$$


where $B_{i}$ is a permissible Cartier divisor with respect to $(X, D)$ and $b_{i} \in \mathbb{R}$ for every $i$.

The reader will find that it is very useful to introduce the notion of globally embedded simple normal crossing pairs for the proof of vanishing and torsion-free theorems (cf. [F7, Chapter 2]).

Definition 2.3 (Globally embedded simple normal crossing pairs). Let $X$ be a simple normal crossing divisor on a smooth variety $M$ and let $B$ be an $\mathbb{R}$-divisor on $M$ such that $\operatorname{Supp}(B+X)$ is a simple normal crossing divisor and that $B$ and $X$ have no common irreducible components. We put $D=\left.B\right|_{X}$ and consider the pair $(X, D)$. We call $(X, D)$ a globally embedded simple normal crossing pair. In this case, it is obvious that $(X, D)$ is an embedded simple normal crossing pair.

In Section 6, we will discuss some vanishing and torsion-free theorems for quasi-projective simple normal crossing pairs, which will play crucial roles in Section 7. See also [F14], [F15], and [F17].

Finally, let us recall the definition of semi divisorial log terminal pairs in the sense of Kollár (see [Ko6, Definition 5.19] and [F14, Definition 4.1]).

Definition 2.4 (Semi divisorial log terminal pairs). Let $X$ be an equidimensional variety which satisfies Serre's $S_{2}$ condition and is normal crossing in codimension one. Let $\Delta$ be a boundary $\mathbb{R}$-divisor on $X$ whose support does not contain any irreducible components of the conductor of $X$. Assume that $K_{X}+\Delta$ is $\mathbb{R}$-Cartier. The pair $(X, \Delta)$ is semi divisorial log terminal if $a(E, X, \Delta)>-1$ for every exceptional divisor $E$ over $X$ such that $(X, \Delta)$ is not a simple normal crossing pair at the generic point of $c_{X}(E)$, where $c_{X}(E)$ is the center of $E$ on $X$.

Remark 2.5. The definition of semi divisorial log terminal pairs in [F1, Definition 1.1] is different from Definition 2.4.

For the details of semi divisorial log terminal pairs, see [Ko6, Section 5.4] and [F14, Section 4].

\section{$\S 3$. Generalities on variation of mixed Hodge structure}

3.1. Let $X$ be a complex analytic variety. For a point $x \in X, \mathbb{C}(x)(\simeq \mathbb{C})$ denotes the residue field at the point $x$. For a morphism $\varphi: \mathcal{F} \longrightarrow \mathcal{G}$ of $\mathcal{O}_{X}$-modules the morphism

$$
\varphi \otimes \mathrm{id}: \mathcal{F} \otimes \mathbb{C}(x) \longrightarrow \mathcal{G} \otimes \mathbb{C}(x)
$$

is denoted by $\varphi(x)$ for a point $x \in X$. 
Remark 3.2. For a complex $K$ equipped with a finite decreasing filtration $F$ and for an integer $q$, the four conditions

(3.2.1) $d: K^{q} \longrightarrow K^{q+1}$ is strictly compatible with the filtration $F$,

(3.2.2) the canonical morphism $H^{q+1}\left(F^{p} K\right) \longrightarrow H^{q+1}(K)$ is injective for all $p$

(3.2.3) the canonical morphism $H^{q+1}\left(F^{p+1} K\right) \longrightarrow H^{q+1}\left(F^{p} K\right)$ is injective for all $p$,

(3.2.4) the canonical morphism $H^{q}\left(F^{p} K\right) \longrightarrow H^{q}\left(\operatorname{Gr}_{F}^{p} K\right)$ is surjective for all $p$

are equivalent. Therefore the strict compatibility in (3.2.1) makes sense in the filtered derived category.

On a complex variety $X$, a complex of $\mathcal{O}_{X}$-modules $K$ is called a perfect complex if, locally on $X$, it is isomorphic in the derived category to a bounded complex consisting of free $\mathcal{O}_{X}$-modules of finite rank (see e.g. [FGAE, 8.3.6.3]). The following definition is an analogue of the notion of perfect complex.

Definition 3.3. Let $X$ be a complex variety. A complex of $\mathcal{O}_{X}$-modules $K$ equipped with a finite decreasing filtration $F$ is called a filtered perfect if $\operatorname{Gr}_{F}^{p} K$ is a perfect complex for all $p$.

Lemma 3.4. Let $X$ be a complex manifold.

(i) For a perfect complex $K$ on $X$, the function

$$
X \ni x \mapsto \operatorname{dim} H^{q}(K \otimes \mathbb{C}(x))
$$

is upper semi-continuous for all $q$.

(ii) Let $K$ be a perfect complex on $X$. If there exists an integer $q_{0}$ such that $H^{q}(K)$ is locally free of finite rank for all $q \geq q_{0}$, then the canonical morphism

$$
H^{q}(K) \otimes \mathcal{F} \longrightarrow H^{q}\left(K \otimes^{L} \mathcal{F}\right)
$$

is an isomorphism for any $\mathcal{O}_{X}$-module $\mathcal{F}$ and for all $q \geq q_{0}$.

(iii) Fix an integer $q$. For a perfect complex $K$ on $X$, the following two conditions are equivalent:

(3.4.1) The function

$$
X \ni x \mapsto \operatorname{dim} H^{q}\left(K \otimes^{L} \mathbb{C}(x)\right)
$$

is locally constant. 
(3.4.2) The sheaf $H^{q}(K)$ is locally free of finite rank and the canonical morphism

$$
H^{q}(K) \otimes \mathcal{F} \longrightarrow H^{q}\left(K \otimes^{L} \mathcal{F}\right)
$$

is an isomorphism for any $\mathcal{O}_{X}$-module $\mathcal{F}$.

Moreover, if these equivalent conditions are satisfied, then the canonical morphism

$$
H^{q-1}(K) \otimes \mathcal{F} \longrightarrow H^{q-1}\left(K \otimes^{L} \mathcal{F}\right)
$$

is an isomorphism for any $\mathcal{O}_{X}$-module $\mathcal{F}$.

(iv) Let $(K, F)$ be a filtered perfect complex on $X$. Assume that the function

$$
X \ni x \mapsto \operatorname{dim} H^{q}\left(K \otimes^{L} \mathbb{C}(x)\right)
$$

is locally constant. If the morphisms

$$
\begin{aligned}
& d(x):\left(K \otimes^{L} \mathbb{C}(x)\right)^{q-1} \longrightarrow\left(K \otimes^{L} \mathbb{C}(x)\right)^{q} \\
& d(x):\left(K \otimes^{L} \mathbb{C}(x)\right)^{q} \longrightarrow\left(K \otimes^{L} \mathbb{C}(x)\right)^{q+1}
\end{aligned}
$$

are strictly compatible with the filtration $F\left(K \otimes^{L} \mathbb{C}(x)\right)$ for every $x \in X$, then $H^{q}\left(\mathrm{Gr}_{F}^{p} K\right)$ is locally free of finite rank, the canonical morphism

$$
H^{q}\left(\operatorname{Gr}_{F}^{p} K\right) \otimes \mathbb{C}(x) \simeq H^{q}\left(\operatorname{Gr}_{F}^{p}\left(K \otimes L{ }^{L} \mathbb{C}(x)\right)\right)
$$

is an isomorphism for all $p$ and for every $x \in X$, and $d: K^{q} \longrightarrow K^{q+1}$ is strictly compatible with the filtration $F$.

Proof. We can easily obtain (i), (ii) and (iii) by the arguments in [Mu, Chapter $5]$.

The strict compatibility conditions in (iv) imply the exactness of the sequence

$$
\begin{aligned}
0 \longrightarrow H^{q}\left(F^{p+1}\left(K \otimes^{L} \mathbb{C}(x)\right)\right) & \longrightarrow H^{q}\left(F^{p}\left(K \otimes^{L} \mathbb{C}(x)\right)\right) \\
& \longrightarrow H^{q}\left(\mathrm{Gr}_{F}^{p} K \otimes^{L} \mathbb{C}(x)\right) \longrightarrow 0
\end{aligned}
$$

for all $p$ and for every $x \in X$. Thus we obtain the equality

$$
\sum_{p} \operatorname{dim} H^{q}\left(\operatorname{Gr}_{F}^{p} K \otimes^{L} \mathbb{C}(x)\right)=\operatorname{dim} H^{q}\left(K \otimes^{L} \mathbb{C}(x)\right)
$$

for every $x$, which implies that $\operatorname{dim} H^{q}\left(\mathrm{Gr}_{F}^{p} K \otimes^{L} \mathbb{C}(x)\right)$ is locally constant with respect to $x \in X$. Applying (iii), $H^{q}\left(\mathrm{Gr}_{F}^{p} K\right)$ is locally free and (3.4.3) is an isomorphism for all $p$ and for any $x \in X$. By using the isomorphisms (3.4.3) for all $p$, we can easily see the surjectivity of the canonical morphism

$$
H^{q}\left(F^{p} K\right) \otimes \mathbb{C}(x) \longrightarrow H^{q}\left(\operatorname{Gr}_{F}^{p} K\right) \otimes \mathbb{C}(x)
$$


for any $x \in X$, and then the canonical morphism

$$
H^{q}\left(F^{p} K\right) \longrightarrow H^{q}\left(\mathrm{Gr}_{F}^{p} K\right)
$$

is surjective for every $p$. Thus the morphism $d: K^{q} \longrightarrow K^{q+1}$ is strictly compatible with the filtration $F$ by Remark 3.2.

Definition 3.5. Let $X$ be a complex manifold. A pre-variation of $\mathbb{Q}$-Hodge structure of weight $m$ on $X$ is a triple $V=(\mathbb{V},(\mathcal{V}, F), \alpha)$ such that

- $\mathbb{V}$ is a local system of finite dimensional $\mathbb{Q}$-vector space on $X$,

- $\mathcal{V}$ is an $\mathcal{O}_{X}$-module and $F$ is a finite decreasing filtration on $\mathcal{V}$,

- $\alpha: \mathbb{V} \longrightarrow \mathcal{V}$ is a morphism of $\mathbb{Q}$-sheaves,

satisfying the conditions

(3.5.1) $\alpha$ induces an isomorphism $\mathcal{O}_{X} \otimes \mathbb{V} \simeq \mathcal{V}$ of $\mathcal{O}_{X}$-modules,

(3.5.2) $\operatorname{Gr}_{F}^{p} \mathcal{V}$ is a locally free $\mathcal{O}_{X}$-module of finite rank for every $p$,

(3.5.3) $\left(\mathbb{V}_{x}, F(\mathcal{V}(x))\right)$ is a Hodge structure of weight $m$ for every $x \in X$, where we identify $\mathbb{V}_{x} \otimes \mathbb{C}$ with $\mathcal{V}(x)$ by the isomorphism $\alpha(x)$.

We denote $\left(\mathbb{V}_{x}, F(\mathcal{V}(x))\right)$ by $V(x)$ for $x \in X$.

We identify $\mathcal{O}_{X} \otimes \mathbb{V}$ with $\mathcal{V}$ by the isomorphism in (3.5.1) if there is no danger of confusion. Under this identification, we write $V=(\mathbb{V}, F)$ for a pre-variation of $\mathbb{Q}$-Hodge structure.

We define the notion of a morphism of pre-variations in the trivial way.

Remark 3.6. A variation of $\mathbb{Q}$-Hodge structure of weight $m$ on $X$ is nothing but a pre-variation $V=(\mathbb{V}, F)$ of $\mathbb{Q}$-Hodge structure of weight $m$ such that the canonical integrable connection $\nabla$ on $\mathcal{V}=\mathcal{O}_{X} \otimes \mathbb{V}$ satisfies the Griffiths transversality

$$
\nabla\left(F^{p}\right) \subset \Omega_{X}^{1} \otimes F^{p-1}
$$

for every $p$. A morphism of variations of $\mathbb{Q}$-Hodge structure is a morphism of underlying pre-variations of $\mathbb{Q}$-Hodge structure.

Remark 3.7. (i) Let $V_{1}=\left(\mathbb{V}_{1}, F\right)$ and $V_{2}=\left(\mathbb{V}_{2}, F\right)$ be pre-variations of $\mathbb{Q}$-Hodge structure of weight $m_{1}$ and $m_{2}$ respectively. Then the local systems $\mathbb{V}_{1} \otimes \mathbb{V}_{2}$ and $\mathcal{H o m}\left(\mathbb{V}_{1}, \mathbb{V}_{2}\right)$ underlie pre-variations of $\mathbb{Q}$-Hodge structure of weight $m_{1}+m_{2}$ and $m_{2}-m_{1}$ respectively. These pre-variations of $\mathbb{Q}$-Hodge structure are denoted by $V_{1} \otimes V_{2}$ and $\mathcal{H o m}\left(V_{1}, V_{2}\right)$ respectively.

(ii) For an integer $n, \mathbb{Q}_{X}(n)$ denotes the pre-variation of $\mathbb{Q}$-Hodge structure of Tate as usual. This is, in fact, a variation of $\mathbb{Q}$-Hodge structure of weight $-2 n$ 
on $X$. For a pre-variation $V$ of $\mathbb{Q}$-Hodge structure of weight $m, V(n)=V \otimes \mathbb{Q}_{X}(n)$ is a pre-variation of $\mathbb{Q}$-Hodge structure of weight $m-2 n$, which is called the Tate twist of $V$ as usual.

Definition 3.8. Let $X$ be a complex manifold and $V=(\mathbb{V}, F)$ a pre-variation of $\mathbb{Q}$-Hodge structure of weight $m$ on $X$. A polarization on $V$ is a morphism of pre-variations of $\mathbb{Q}$-Hodge structure

$$
V \otimes V \longrightarrow \mathbb{Q}_{X}(-m)
$$

which induces a polarization on $V(x)$ for every point $x \in X$. A pre-variation of $\mathbb{Q}$ Hodge structure of weight $m$ is said to be polarizable, if there exists a polarization on it. A morphism of polarizable pre-variations of $\mathbb{Q}$-Hodge structure is a morphism of the underlying pre-variations of $\mathbb{Q}$-Hodge structure.

Definition 3.9. Let $X$ be a complex manifold.

(i) A pre-variation of $\mathbb{Q}$-mixed Hodge structure on $X$ is a triple

$$
V=((\mathbb{V}, W),(\mathcal{V}, W, F), \alpha)
$$

consisting of

- a local system of finite dimensional $\mathbb{Q}$-vector space $\mathbb{V}$, equipped with a finite increasing filtration $W$ by local subsystems,

- an $\mathcal{O}_{X}$-module $\mathcal{V}$ equipped with a finite increasing filtration $W$ and a finite decreasing filtration $F$,

- a morphism of $\mathbb{Q}$-sheaves $\alpha: \mathbb{V} \longrightarrow \mathcal{V}$ preserving the filtration $W$

such that the triple $\mathrm{Gr}_{m}^{W} V=\left(\mathrm{Gr}_{m}^{W} \mathbb{V},\left(\mathrm{Gr}_{m}^{W} \mathcal{V}, F\right), \mathrm{Gr}_{m}^{W} \alpha\right)$ is a pre-variation of $\mathbb{Q}$-Hodge structure of weight $m$ for every $m$.

We identify $\left(\mathcal{O}_{X} \otimes \mathbb{V}, W\right)$ and $(\mathcal{V}, W)$ by the isomorphism induced by $\alpha$ as before, if there is no danger of confusion. Under this identification, we use the notation $V=(\mathbb{V}, W, F)$ for a pre-variation of $\mathbb{Q}$-mixed Hodge structure.

(ii) A pre-variation $V=(\mathbb{V}, W, F)$ of $\mathbb{Q}$-mixed Hodge structure on $X$ is called graded polarizable, if $\mathrm{Gr}_{m}^{W} V$ is a polarizable pre-variation of $\mathbb{Q}$-Hodge structure for every $m$.

(iii) We define the notion of a morphism of pre-variations of $\mathbb{Q}$-mixed Hodge structure by the trivial way. A morphism of polarizable pre-variations of $\mathbb{Q}$-mixed Hodge structure is a morphism of the underlying pre-variations.

Now, let us recall the definition of graded polarizable variation of $\mathbb{Q}$-mixed Hodge structure (GPVMHS, for short). See, for example, [SZ, §3], [SSU, Part I, Section 1], [BZ, Section 7], [PS, Definitions 14.44 and 14.45], and so on. 
Definition 3.10 (GPVMHS). Let $X$ be a complex manifold.

(i) A pre-variation $V=(\mathbb{V}, W, F)$ of $\mathbb{Q}$-mixed Hodge structure on $X$ is said to be a variation of $\mathbb{Q}$-mixed Hodge structure, if the canonical integrable connection $\nabla$ on $\mathcal{V} \simeq \mathcal{O}_{X} \otimes \mathbb{V}$ satisfies the Griffiths transversality (3.6.1) in Remark 3.6.

(ii) A variation of $\mathbb{Q}$-mixed Hodge structure is called graded polarizable, if the underlying pre-variation is graded polarizable.

(iii) A morphism of (graded polarizable) variations of $\mathbb{Q}$-mixed Hodge structure is a morphism of the underlying pre-variations.

The following definition of the admissibility is given by Steenbrink-Zucker [SZ, (3.13) Properties] in the one-dimensional case and by Kashiwara [Ks, 1.8, 1.9] in the general case. See also [PS, Definition 14.49].

Definition 3.11 (Admissibility (cf. $[\mathrm{Ks}, 1.8,1.9]$ )). (i) A variation of $\mathbb{Q}$-mixed Hodge structure $V=(\mathbb{V}, W, F)$ over $\Delta^{*}=\Delta \backslash\{0\}$, where $\Delta=\{t \in \mathbb{C}|| t \mid<1\}$, is said to be pre-admissible if it satisfies:

(3.11.1) The monodromy around the origin is quasi-unipotent.

(3.11.2) Let $\widetilde{\mathcal{V}}$ and $W_{k} \widetilde{\mathcal{V}}$ be the upper canonical extensions of $\mathcal{V}=\mathcal{O}_{\Delta^{*}} \otimes \mathbb{V}$ and of $\mathcal{O}_{\Delta^{*}} \otimes W_{k} \mathbb{V}$ in the sense of Deligne [D1, Remarques 5.5 (i)] (see also [Ko2, Section 2] and Remark 7.4). Then the filtration $F$ on $\mathcal{V}$ extends to the filtration $F$ on $\widetilde{\mathcal{V}}$ such that $\operatorname{Gr}_{F}^{p} \mathrm{Gr}_{k}^{W} \widetilde{\mathcal{V}}$ is locally free $\mathcal{O}_{\Delta}$-modules of finite rank for each $k, p$.

(3.11.3) The logarithm of the unipotent part of the monodromy admits a weight filtration relative to $W$.

(ii) Let $X$ be a complex variety and $U$ a nonsingular Zariski open subset of $X$. A variation of $\mathbb{Q}$-mixed Hodge structure $V$ on $U$ is said to be admissible (with respect to $X)$ if for every morphism $i: \Delta \longrightarrow X$ with $i\left(\Delta^{*}\right) \subset U$, the variation $i^{*} V$ on $\Delta^{*}$ is pre-admissible.

We can define an $\mathbb{R}$-mixed Hodge structure, a variation of $\mathbb{R}$-mixed Hodge structure, and so on, analogously.

We frequently use the following lemma in Section 5, which is a special case of [Ks, Proposition 1.11.3] (see also Remark 7.4 below).

Proposition 3.12 (cf. $[\mathrm{Ks}]$ ). Let $X$ be a complex manifold, $U$ the complement of a normal crossing divisor on $X$ and $V=(\mathbb{V}, W, F)$ a variation of $\mathbb{R}$-mixed Hodge structure on $U$. The upper canonical extensions of $\mathcal{V}=\mathcal{O}_{U} \otimes \mathbb{V}$ and of $W_{k} \mathcal{V}=\mathcal{O}_{U} \otimes W_{k} \mathbb{V}$ are denoted by $\widetilde{\mathcal{V}}$ and by $W_{k} \widetilde{\mathcal{V}}$ respectively. If $V$ is admissible on $U$ with respect to $X$, then the filtration $F$ on $\mathcal{V}$ extends to a finite filtration $F$ 
on $\widetilde{\mathcal{V}}$ by subbundles such that $\operatorname{Gr}_{F}^{p} \operatorname{Gr}_{k}^{W} \widetilde{\mathcal{V}}$ is a locally free $\mathcal{O}_{X}$-module of finite rank for all $k, p$.

We give an elementary but useful remark on the quasi-unipotency of monodromy.

Remark 3.13 (Quasi-unipotency). If the local system $\mathbb{V}$ has a $\mathbb{Z}$-structure, that is, there is a local system $\mathbb{V}_{\mathbb{Z}}$ on $X$ of $\mathbb{Z}$-modules of finite rank such that $\mathbb{V}=\mathbb{V}_{\mathbb{Z}} \otimes \mathbb{Q}$, in Definition 3.11, then the quasi-unipotency automatically follows from Borel's theorem (cf. [Sc, (4.5) Lemma (Borel)]).

The following lemma states the fundamental results on pre-variations of $\mathbb{Q}$ Hodge structure.

Lemma 3.14. Let $X$ be a complex manifold.

(i) The category of the pre-variations of $\mathbb{Q}$-Hodge structure of weight $m$ on $X$ is an abelian category for every $m$.

(ii) Let $V_{1}$ and $V_{2}$ be pre-variations of $\mathbb{Q}$-Hodge structure of weight $m_{1}$ and $m_{2}$ respectively, and $\varphi: V_{1} \longrightarrow V_{2}$ a morphism of pre-variations. If $m_{1}>m_{2}$, then $\varphi=0$.

(iii) Let $\varphi: V_{1} \longrightarrow V_{2}$ be a morphism of pre-variations $V_{1}=\left(\mathbb{V}_{1}, F\right)$ and $V_{2}=\left(\mathbb{V}_{2}, F\right)$ of $\mathbb{Q}$-Hodge structure of weight $m$ on $X$. Then the induced morphism $\varphi \otimes \mathrm{id}: \mathbb{V}_{1} \otimes \mathcal{O}_{X} \longrightarrow \mathbb{V}_{2} \otimes \mathcal{O}_{X}$ is strictly compatible with the filtration $F$.

(iv) The functor from the category of the pre-variations of $\mathbb{Q}$-Hodge structure of weight $m$ to the category of the $\mathbb{Q}$-Hodge structures of weight $m$ which assigns $V$ to $V(x)$ is an exact functor for every $x \in X$.

$(\mathrm{v})$ The category of the polarizable variations of $\mathbb{Q}$-Hodge structure of weight $m$ on $X$ is an abelian category for every $m$.

Proof. The statements (i), (iii) and (iv) are easy consequences of Lemma 3.4 (iv), and (ii) is easily proved by the corresponding result for $\mathbb{Q}$-Hodge structures. So we prove $(\mathrm{v})$ now.

Let $V_{1}=\left(\mathbb{V}_{1}, F\right)$ and $V_{2}=\left(\mathbb{V}_{2}, F\right)$ be polarizable pre-variations of $\mathbb{Q}$-Hodge structure of weight $m$ on $X$, and $\varphi: V_{1} \longrightarrow V_{2}$ a morphism. We fix polarizations on $V_{1}$ and $V_{2}$ respectively. Taking (i) into the account, it is sufficient to prove that $\operatorname{Ker}(\varphi)$ and $\operatorname{Coker}(\varphi)$ are polarizable. The case of $\operatorname{Ker}(\varphi)$ is trivial. Then we discuss the case of $\operatorname{Coker}(\varphi)$.

The morphism $\varphi$ induces a morphism

$$
\varphi^{*}: \mathcal{H o m}\left(V_{2}, \mathbb{Q}_{X}(-m)\right) \longrightarrow \mathcal{H} o m\left(V_{1}, \mathbb{Q}_{X}(-m)\right)
$$


which is clearly a morphism of pre-variations of $\mathbb{Q}$-Hodge structure of weight $m$. On the other hand, the polarizations on $V_{1}$ and $V_{2}$ induce the identifications

$$
V_{1} \simeq \mathcal{H o m}\left(V_{1}, \mathbb{Q}_{X}(-m)\right), \quad V_{2} \simeq \mathcal{H} o m\left(V_{2}, \mathbb{Q}_{X}(-m)\right)
$$

which are isomorphisms of pre-variations of $\mathbb{Q}$-Hodge structure. By these identifications the morphism $\varphi^{*}$ above can be considered as a morphism of pre-variations $V_{2} \longrightarrow V_{1}$, which is denoted by the same symbol $\varphi^{*}$ by abuse of the language. Then the inclusion $\operatorname{Ker}\left(\varphi^{*}\right) \hookrightarrow V_{2}$ induces an isomorphism $\operatorname{Ker}\left(\varphi^{*}\right) \simeq \operatorname{Coker}(\varphi)$ of pre-variations. Therefore we obtain a polarization expected.

Here we give a brief remark on the dual of a variation of $\mathbb{Q}$-mixed Hodge structure.

Remark 3.15. Let $V=((\mathbb{V}, W),(\mathcal{V}, W, F), \alpha)$ be a pre-variation of $\mathbb{Q}$-mixed Hodge structure on a complex manifold $X$. On the dual local system $\mathbb{V}^{*}=$ $\mathcal{H o m}_{\mathbb{Q}}(\mathbb{V}, \mathbb{Q})$,

$$
W_{m} \mathbb{V}^{*}=\left(\mathbb{V} / W_{-m-1}\right)^{*} \subset \mathbb{V}^{*}
$$

defines an increasing filtration $W$. Similarly, on $\mathcal{V}^{*}=\mathcal{H}_{o} m_{\mathcal{O}_{X}}\left(\mathcal{V}, \mathcal{O}_{X}\right)$

$$
W_{m} \mathcal{V}^{*}=\left(\mathcal{V} / W_{-m-1}\right)^{*}, \quad F^{p} \mathcal{V}^{*}=\left(\mathcal{V} / F^{1-p}\right)^{*}
$$

define increasing and decreasing filtrations on $\mathcal{V}^{*}$. We have

$$
\operatorname{Gr}_{F}^{p} \operatorname{Gr}_{m}^{W} \mathcal{V}^{*} \simeq\left(\operatorname{Gr}_{F}^{-p} \operatorname{Gr}_{-m}^{W} \mathcal{V}\right)^{*}
$$

for every $m, p$ by definition. Equipped with an isomorphism $\mathcal{O}_{X} \otimes \mathbb{V}^{*} \simeq \mathcal{V}^{*}$, it turns out that $\left(\left(\mathbb{V}^{*}, W\right),\left(\mathcal{V}^{*}, W, F\right)\right)$ is a pre-variation of $\mathbb{Q}$-mixed Hodge structure on $X$. It is denoted by $V^{*}$ for short and is called the dual of $V$. It is easy to see that $V^{*}$ is graded polarizable or a variation of $\mathbb{Q}$-mixed Hodge structure if $V$ is so. For the case where $X$ is a Zariski open subset of another variety, $V^{*}$ is admissible if $V$ is so.

We close this section by the lemma concerning with the relative monodromy weight filtration for a filtered $\mathbb{Q}$-mixed Hodge complex. For the definition, see, for example, [E2, 6.1.4 Définition].

Remark 3.16. Now we remark the notation on the shift of increasing filtrations.

We set

$$
W[m]_{k}=W_{k-m}
$$

as in [D2], [E2]. Our notation is different from the one in [CKS]. 
Lemma 3.17. Let $\left(\left(A_{\mathbb{Q}}, W^{f}, W\right),\left(A_{\mathbb{C}}, W^{f}, W, F\right), \alpha\right)$ be a filtered $\mathbb{Q}$-mixed Hodge complex such that the spectral sequence $E_{r}^{p, q}\left(A_{\mathbb{C}}, W^{f}\right)$ degenerates at $E_{2}$-terms, and $\nu: A_{\mathbb{C}} \longrightarrow A_{\mathbb{C}}$ a morphism of complexes preserving the filtration $W^{f}$ and satisfying the condition $\nu\left(W_{m} A_{\mathbb{C}}\right) \subset W_{m-2} A_{\mathbb{C}}$ for every $m$. If the filtration $W[-m]$ on $H^{n}\left(\mathrm{Gr}_{m}^{W^{f}} A_{\mathbb{C}}\right)$ is the monodromy weight filtration of the endomorphism $H^{n}\left(\mathrm{Gr}_{m}^{W^{f}} \nu\right)$ for all $m, n$, then the filtration $W$ on $H^{n}\left(A_{\mathbb{C}}\right)$ is the relative weight monodromy filtration of the endomorphism $H^{n}(\nu)$ with respect to the filtration $W^{f}$ for all $n$.

Proof. The assumption implies that the morphism $H^{p+q}\left(\mathrm{Gr}_{-p}^{W^{f}} \nu\right)^{k}$ induces an isomorphism

$$
\operatorname{Gr}_{q+k}^{W[p+q]} E_{1}^{p, q}\left(A_{\mathbb{C}}, W^{f}\right) \longrightarrow \operatorname{Gr}_{q-k}^{W[p+q]} E_{1}^{p, q}\left(A_{\mathbb{C}}, W^{f}\right)
$$

for all $p, q$ and for $k \geq 0$ because of the isomorphism $E_{1}^{p, q}\left(A_{\mathbb{C}}, W^{f}\right) \simeq H^{p+q}\left(\mathrm{Gr}_{-p}^{W^{f}} A_{\mathbb{C}}\right)$. On the other hand, the $E_{2}$-degeneracy for the filtration $W^{f}$ gives us the isomorphism

$$
E_{2}^{p, q}\left(A_{\mathbb{C}}, W^{f}\right) \simeq \mathrm{Gr}_{-p}^{W^{f}} H^{p+q}\left(A_{\mathbb{C}}\right)
$$

for all $p, q$, under which the filtration $W_{\text {rec }}$ on the left hand side coincides with the filtration $W$ on the right hand side by [E2, 6.1.8 Théorème]. Since the morphism $d_{1}$ of $E_{1}$-terms induces a morphism of mixed Hodge structures

$$
d_{1}:\left(E_{1}^{p, q}\left(A_{\mathbb{C}}, W^{f}\right), W[p+q], F\right) \longrightarrow\left(E_{1}^{p+1, q}\left(A_{\mathbb{C}}, W^{f}\right), W[p+q+1], F\right)
$$

for all $p, q$ by [E2, 6.1.8 Théorème] again, the morphism $\left(H^{p+q}(\nu)\right)^{k}$ induces an isomorphism

$$
\mathrm{Gr}_{q+k}^{W[p+q]} \operatorname{Gr}_{-p}^{W^{f}} H^{p+q}\left(A_{\mathbb{C}}\right) \longrightarrow \operatorname{Gr}_{q-k}^{W[p+q]} \operatorname{Gr}_{-p}^{W^{f}} H^{p+q}\left(A_{\mathbb{C}}\right)
$$

for $p, q$ and for $k \geq 0$. Then we can easily check the conclusion.

\section{§4. Variation of mixed Hodge structure of geometric origin}

In this section, we discuss variations of mixed Hodge structure arising from mixed Hodge structures on cohomology with compact support for simple normal crossing pairs. We will check that those variations of mixed Hodge structure are graded polarizable and admissible (see Theorem 4.15). These properties will play crucial roles in the subsequent sections.

4.1. For a morphism of topological spaces $f: X \longrightarrow Y$, we always use the Godment resolution to compute the higher direct image $R f_{*}$ of abelian sheaves on $X$. This means that $R f_{*} \mathcal{F}$ is the genuine complex $f_{*} \mathcal{C}_{\text {Gdm }}^{\bullet} \mathcal{F}$ for an abelian sheaf $\mathcal{F}$ on $X$, where $\mathcal{C}_{\text {Gdm }}^{\bullet}$ stands for the Godment resolution as in Peters-Steenbrink 
[PS, B.2.1]. If $\mathcal{F}$ carries a filtration $F, R f_{*} \mathcal{F}$ is the genuine filtered complex. For a morphism of sheaves $\varphi: \mathcal{F} \longrightarrow \mathcal{G}$ on $X$, the morphism

$$
R f_{*}(\varphi): R f_{*} \mathcal{F} \longrightarrow R f_{*} \mathcal{G}
$$

is the genuine morphism of complexes defined by using the Godment resolution. We use the same notation for complexes of abelian sheaves, $\mathbb{Q}$-sheaves, $\mathbb{C}$-sheaves, $\mathcal{O}_{X}$-modules and so on.

4.2. Let $f: X_{\bullet} \longrightarrow Y$ be an augmented semi-simplicial topological space. The morphism $X_{p} \longrightarrow Y$ induced by $f$ is denoted by $f_{p}$ for every $p$. For an abelian sheaf $\mathcal{F}^{\bullet}$ on $X_{\bullet}$.

$$
R f_{\bullet *} \mathcal{F}^{\bullet}=\left\{R f_{p *} \mathcal{F}^{p}\right\}_{p \geq 0}
$$

defines a co-semi-simplicial complexes on $Y$ by what we mentioned in 4.1. Then we define

$$
R f_{*} \mathcal{F}^{\bullet}=s R f_{\bullet *} \mathcal{F}^{\bullet}
$$

as in $[\mathrm{D} 3,(5.2 .6 .1)]$. More precisely, $R f_{*} \mathcal{F}^{\bullet}$ is the single complex associated to the double complex

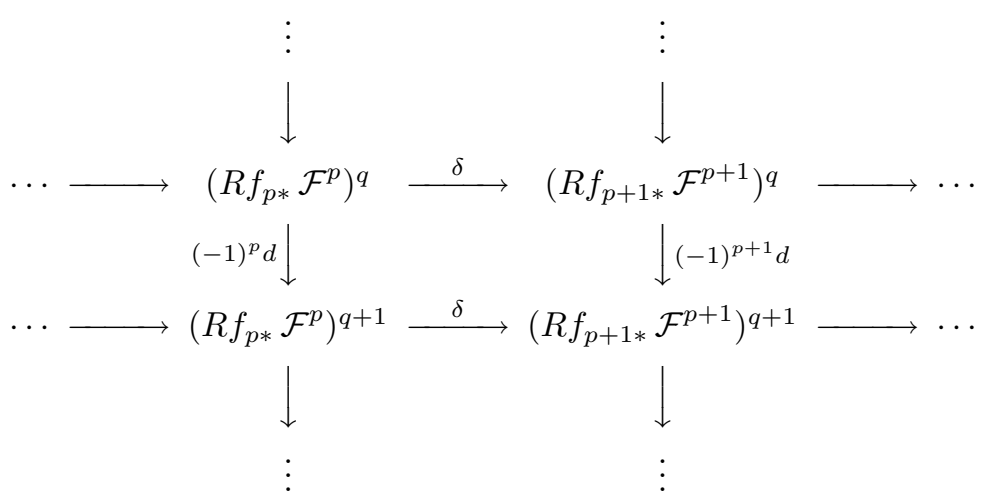

where $\delta$ in the horizontal lines denotes the Čech type morphism and $d$ in the vertical lines denotes the differential of the complexes $R f_{p *} \mathcal{F}^{p}$ for every $p$. The increasing filtration $L$ on $R f_{*} \mathcal{F}^{\bullet}$ is defined by

$$
L_{m}\left(R f_{*} \mathcal{F}^{\bullet}\right)^{n}=\bigoplus_{p \geq-m}\left(R f_{p *} \mathcal{F}^{p}\right)^{n-p}
$$

for every $m, n$ (cf. Deligne [D3, (5.1.9.3)]). Thus we have

$$
\operatorname{Gr}_{m}^{L} R f_{*} \mathcal{F}^{\bullet}=R f_{-m *} \mathcal{F}^{-m}[m]
$$


for every $m$. For the case where $\mathcal{F}^{\bullet}$ admits an increasing filtration $W$, we set

$$
\begin{aligned}
& W_{m}\left(R f_{*} \mathcal{F}^{\bullet}\right)^{n}=\bigoplus_{p} W_{m}\left(R f_{p *} \mathcal{F}^{p}\right)^{n-p} \\
& \delta(W, L)_{m}\left(R f_{*} \mathcal{F}^{\bullet}\right)^{n}=\bigoplus_{p} W_{m+p}\left(R f_{p *} \mathcal{F}^{p}\right)^{n-p}
\end{aligned}
$$

for every $n, p$. Then we have

$$
\operatorname{Gr}_{m}^{\delta(W, L)} R f_{*} \mathcal{F}=\bigoplus_{p} \operatorname{Gr}_{m+p}^{W} R f_{p *} \mathcal{F}^{p}[-p]
$$

for every $m$. The case of decreasing filtration $F$ on $\mathcal{F}$ is transformed to the case of increasing filtration by setting $W_{m} \mathcal{F}=F^{-m} \mathcal{F}$. We use the same convention for complexes of abelian sheaves, $\mathbb{Q}$-sheaves and so on.

4.3. Let $X$ and $Y$ be complex manifolds and $f: X \longrightarrow Y$ a smooth projective morphism. The de Rham complexes of $X$ and $Y$ are denoted by $\Omega_{X}$ and $\Omega_{Y}$ respectively, and the relative de Rham complex for the morphism $f$ is denoted by $\Omega_{X / Y}$. Moreover, $F$ denotes the stupid filtration on $\Omega_{X}$ and $\Omega_{X / Y}$. The inclusion $\mathbb{Q}_{X} \longrightarrow \mathcal{O}_{X}$ induces a morphism of complexes $\mathbb{Q}_{X} \longrightarrow \Omega_{X / Y}$. Then we obtain a morphism

$$
R^{i} f_{*} \mathbb{Q}_{X} \longrightarrow R^{i} f_{*} \Omega_{X / Y}
$$

for every $i$, which is denoted by $\alpha_{X / Y}$ simply. Then

$$
\left(R^{i} f_{*} \mathbb{Q}_{X},\left(R^{i} f_{*} \Omega_{X / Y}, F\right), \alpha_{X / Y}\right)
$$

is a polarizable variation of $\mathbb{Q}$-Hodge structure of weight $i$ on $Y$. Here we recall the proof of the Griffiths transversality following [KO].

A finite decreasing filtration $G$ on $\Omega_{X}$ is defined by

$$
G^{p} \Omega_{X}=\operatorname{Im}\left(f^{-1} \Omega_{Y}^{p} \otimes_{f^{-1} \mathcal{O}_{Y}} \Omega_{X}[-p] \longrightarrow \Omega_{X}\right)
$$

for all $p$. Then we have isomorphisms

$$
\operatorname{Gr}_{G}^{p} \Omega_{X} \simeq f^{-1} \Omega_{Y}^{p} \otimes_{f^{-1} \mathcal{O}_{Y}} \Omega_{X / Y}[-p]
$$

for all $p$, which induces isomorphisms

$$
E_{1}^{p, q}\left(R f_{*} \Omega_{X}, G\right) \simeq \Omega_{Y}^{p} \otimes R^{q} f_{*} \Omega_{X / Y}
$$

for every $p, q$. Thus the morphisms of the $E_{1}$-terms give us

$$
\nabla: \Omega_{Y}^{p} \otimes R^{q} f_{*} \Omega_{X / Y} \longrightarrow \Omega_{Y}^{p+1} \otimes R^{q} f_{*} \Omega_{X / Y}
$$


for all $p, q$. It is easy to see that

$$
\nabla: R^{q} f_{*} \Omega_{X / Y} \longrightarrow \Omega_{Y}^{1} \otimes R^{q} f_{*} \Omega_{X / Y}
$$

satisfies the Griffiths transversality.

On the other hand, we consider the complexes $\Omega_{Y}$ and $f^{-1} \Omega_{Y}$. The stupid filtration on $\Omega_{Y}$ is denoted by $G$ for a while. We have

$$
\begin{aligned}
\operatorname{Gr}_{G}^{p} f^{-1} \Omega_{Y} & \simeq f^{-1} \Omega_{Y}^{p}[-p] \\
& =f^{-1} \Omega_{Y}^{p} \otimes_{\mathbb{Q}} \mathbb{Q}_{X}[-p] \\
& =f^{-1} \Omega_{Y}^{p} \otimes_{f^{-1} \mathcal{O}_{Y}} f^{-1} \mathcal{O}_{Y}[-p]
\end{aligned}
$$

for every $p$. Therefore the $E_{1}$-terms of the associated spectral sequence is identified with

$$
E_{1}^{p, q}\left(R f_{*} f^{-1} \Omega_{Y}, G\right)=\Omega_{Y}^{p} \otimes_{\mathbb{Q}} R^{q} f_{*} \mathbb{Q}_{X}
$$

under which the morphisms of $E_{1}$-terms are identified with

$$
d \otimes \mathrm{id}: \Omega_{Y}^{p} \otimes_{\mathbb{Q}} R^{q} f_{*} \mathbb{Q}_{X} \longrightarrow \Omega_{Y}^{p+1} \otimes_{\mathbb{Q}} R^{q} f_{*} \mathbb{Q}_{X}
$$

for every $p, q$. On the other hand, the canonical morphism

$$
f^{-1} \Omega_{Y} \longrightarrow \Omega_{X}
$$

is a filtered quasi-isomorphism by the relative Poincaré lemma. Thus we obtain a commutative diagram

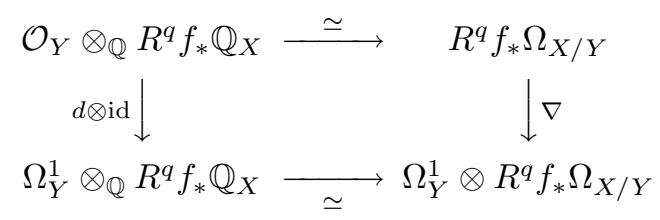

which shows that $d \otimes$ id on $\mathcal{O}_{Y} \otimes_{\mathbb{Q}} R^{i} f_{*} \mathbb{Q}_{X}$ satisfies the Griffiths transversality.

Notation 4.4. A semi-simplicial variety $X_{\bullet}$ is said to be strict if there exists a non-negative integer $p_{0}$ such that $X_{p}=\emptyset$ for all $p \geq p_{0}$.

For an augmented semi-simplicial variety $f: X_{\bullet} \longrightarrow Y$, we say $f$ is smooth, projective etc., if $f_{p}: X_{p} \longrightarrow Y$ is smooth, projective etc. for all $p$.

Lemma 4.5. Let $f: X_{\bullet} \longrightarrow Y$ be a smooth projective augmented strict semisimplicial variety. Moreover, we assume that $Y$ is smooth. Then $R^{i} f_{*} \mathbb{Q}_{X}$. underlies a graded polarizable variation of $\mathbb{Q}$-mixed Hodge structure on $Y$ for all $i$.

Proof. The morphism

$$
R^{i} f_{*}\left(\alpha_{X \bullet / Y}\right): R^{i} f_{*} \mathbb{Q}_{X} \longrightarrow R^{i} f_{*} \Omega_{X \bullet / Y}
$$


is induced by the canonical morphism $\alpha_{X_{\bullet} / Y}: \mathbb{Q}_{X} \longrightarrow \Omega_{X_{\bullet} / Y}$. We can easily see that $R^{i} f_{*}\left(\alpha_{X_{\bullet} / Y}\right)$ induces the isomorphism

$$
R^{i} f_{*} \mathbb{Q}_{X \bullet / Y} \otimes \mathcal{O}_{Y} \longrightarrow R^{i} f_{*} \Omega_{X \bullet / Y}
$$

by the relative Poincaré lemma.

The filtration $L$ on $R^{i} f_{*} \mathbb{Q}_{X}$. and $R^{i} f_{*} \Omega_{X \bullet / Y}$ is defined by (4.2.1). Moreover, the stupid filtration $F$ on $\Omega_{X_{\bullet} / Y}$ induces the filtration $F$ on $R^{i} f_{*} \Omega_{X \cdot / Y}$ by the same way as (4.2.3). It is sufficient to prove that

$$
\left(\left(R^{i} f_{*} \mathbb{Q}_{X_{\bullet}}, L[i]\right),\left(R^{i} f_{*} \Omega_{X \bullet / Y}, L[i], F\right), R^{i} f_{*}\left(\alpha_{X \bullet / Y}\right)\right)
$$

is a graded polarizable variation of $\mathbb{Q}$-mixed Hodge structure on $Y$.

To this end, we consider the data

$$
K=\left(\left(R f_{*} \mathbb{Q}_{X_{\bullet}}, L\right),\left(R f_{*} \Omega_{X_{\bullet} / Y}, L, F\right), R f_{*}\left(\alpha_{X_{\bullet} / Y}\right)\right)
$$

and the spectral sequence associated to the filtration $L$. By (4.2.2), we have

$$
E_{1}^{p, q}(K, L)=\left(R^{q} f_{p *} \mathbb{Q}_{X_{p}},\left(R^{q} f_{p *} \Omega_{X_{p} / Y}, F\right), R^{q} f_{p *}\left(\alpha_{X_{p} / Y}\right)\right),
$$

which is a polarizable variation of $\mathbb{Q}$-Hodge structure of weight $q$ for every $p, q$. Moreover, the morphism

$$
d_{1}: E_{1}^{p, q}(K, L) \longrightarrow E_{1}^{p+1, q}(K, L)
$$

is a morphism of variations of $\mathbb{Q}$-Hodge structure. Therefore $E_{2}^{p, q}(K, L)$ is a polarizable variation of $\mathbb{Q}$-Hodge structure of weight $q$ for every $p, q$ and $F_{\text {rec }}=F_{d}=F_{d^{*}}$ on $E_{2}^{p, q}(K, L)$ by the lemma on two filtrations in [D3]. Then the morphism

$$
d_{2}: E_{2}^{p, q}(K, L) \longrightarrow E_{2}^{p+2, q-1}(K, L)
$$

between $E_{2}$-terms is a morphism of variations of $\mathbb{Q}$-Hodge structure of weight $q$ and of weight $q-1$ respectively, which implies that $d_{2}=0$ (see Lemma 3.14 (ii)). Therefore the spectral sequence $E_{r}^{p, q}(K, L)$ degenerates at $E_{2}$-terms and $F=$ $F_{\text {rec }}=F_{d}=F_{d^{*}}$ on $E_{\infty}^{p, q}(K, L)=\mathrm{Gr}_{-p}^{L} H^{p+q}(K)$ by the lemma on two filtrations again. Thus it turns out that

$$
\operatorname{Gr}_{m}^{L[i]} H^{i}(K)=\left(\operatorname{Gr}_{m}^{L[i]} R^{i} f_{*} \mathbb{Q}_{X_{\bullet}},\left(\operatorname{Gr}_{m}^{L[i]} R^{i} f_{*} \Omega_{X \bullet} / Y, F\right), \operatorname{Gr}_{m}^{L[i]} R^{i} f_{*}\left(\alpha_{X} / Y\right)\right)
$$

is a polarizable pre-variation of $\mathbb{Q}$-Hodge structure of weight $m$ on $Y$ for every $i, m$.

What remains to prove is that the morphism

$$
d \otimes \mathrm{id}: \mathcal{O}_{Y} \otimes R^{i} f_{*} \mathbb{Q}_{X} \longrightarrow \Omega_{Y}^{1} \otimes R^{i} f_{*} \mathbb{Q}_{X}
$$


satisfies the Griffiths transversality under the identification (4.5.1).

Now we consider $R f_{*} f_{\bullet}^{-1} \Omega_{Y}$ and $R f_{*} \Omega_{X \bullet}$, where $f_{\bullet}^{-1} \Omega_{Y}$ denotes the complex $\left\{f_{p}^{-1} \Omega_{Y}\right\}_{p \geq 0}$ on $X_{\bullet}$. The filtration $G$ on $\Omega_{Y}$ and $\Omega_{X}$. induces the filtration $G$ on $R f_{*} f_{\bullet}^{-1} \Omega_{Y}$ and $R f_{*} \Omega_{X}$. Moreover, the filtration $F$ on $\Omega_{X \bullet}$ induces the filtration $F$ on $R f_{*} \Omega_{X}$. The canonical morphism

$$
\gamma: f_{\bullet}^{-1} \Omega_{Y} \longrightarrow \Omega_{X}
$$

which is a filtered quasi-isomorphism with respect to the filtration $G$ by the relative Poincaré lemma, induces the filtered quasi-isomorphism

$$
R f_{*}(\gamma): R f_{*} f_{\bullet}^{-1} \Omega_{Y} \longrightarrow R f_{*} \Omega_{X}
$$

with respect to $G$. Now we consider the spectral sequences associated to $G$, and the morphism of the spectral sequences induced by $R f_{*}(\gamma)$.

We have

$$
\mathrm{Gr}_{G}^{p} R f_{*} f_{\bullet}^{-1} \Omega_{Y} \simeq R f_{*} f_{\bullet}^{-1} \Omega_{Y}^{p}[-p] \simeq \Omega_{Y}^{p} \otimes R f_{*} \mathbb{Q}_{X}[-p]
$$

and

$$
\begin{aligned}
\operatorname{Gr}_{G}^{p} R f_{*} \Omega_{X} & \simeq R f_{*}\left(f_{\bullet}^{-1} \Omega_{Y}^{p} \otimes \Omega_{X \bullet / Y}[-p]\right) \\
& \simeq \Omega_{Y}^{p} \otimes R f_{*} \Omega_{X_{\bullet} / Y}[-p]
\end{aligned}
$$

for every $p$, such that the diagram

$$
\begin{array}{rlr}
\operatorname{Gr}_{G}^{p} R f_{*} f_{\bullet}^{-1} \Omega_{Y} & \simeq \Omega_{Y}^{p} \otimes R f_{*} \mathbb{Q}_{X}[-p] \\
\operatorname{Gr}_{G}^{p} R f_{*}(\gamma) \downarrow & \downarrow \\
\operatorname{Gr}_{G}^{p} R f_{*} \Omega_{X \bullet} & & \simeq \operatorname{id} \otimes R f_{*}\left(\alpha_{X \bullet / Y}\right)[-p] \\
& \simeq \Omega_{Y}^{p} \otimes R f_{*} \Omega_{X \bullet / Y}[-p]
\end{array}
$$

is commutative.

The morphism

$$
\nabla: R^{i} f_{*} \Omega_{X \bullet / Y} \longrightarrow \Omega_{Y}^{1} \otimes R^{i} f_{*} \Omega_{X \bullet / Y}
$$

is induced by the morphism of $E_{1}$-terms

$$
d_{1}: E_{1}^{0, i}\left(R f_{*} \Omega_{X \bullet}, G\right) \longrightarrow E_{1}^{1, i}\left(R f_{*} \Omega_{X \bullet}, G\right)
$$

via the identification (4.5.4). On the other hand, the morphism of $E_{1}$-terms

$$
d_{1}: E_{1}^{0, i}\left(R f_{*} f_{\bullet}^{-1} \Omega_{Y}, G\right) \longrightarrow E_{1}^{1, i}\left(R f_{*} f_{\bullet}^{-1} \Omega_{Y}, G\right)
$$

is identified with

$$
d \otimes \mathrm{id}: \mathcal{O}_{Y} \otimes R^{i} f_{*} \mathbb{Q}_{X} \longrightarrow \Omega_{Y}^{1} \otimes R^{i} f_{*} \mathbb{Q}_{X} .
$$


by the isomorphism (4.5.3). By the commutativity of the diagram (4.5.5), the morphisms $\nabla$ and $d \otimes$ id are identified under the isomorphism (4.5.1). Because $\nabla$ satisfies the Griffiths transversality, so does $d \otimes \mathrm{id}$.

Remark 4.6. The spectral sequence $E_{r}^{p, q}\left(R f_{*} \Omega_{X \cdot / Y}, F\right)$ degenerates at $E_{1}$-terms by the lemma on two filtrations [D3, Proposition (7.2.8)].

Remark 4.7. The construction above is functorial and compatible with the pullback by the morphism $Y^{\prime} \longrightarrow Y$.

Lemma 4.8. Let $f: X_{\bullet} \longrightarrow Y$ and $g: Z_{\bullet} \longrightarrow Y$ be smooth projective augmented strict semi-simplicial varieties and $\varphi: Z_{\bullet} \longrightarrow X_{\bullet}$ a morphism of semi-simplicial varieties compatible with the augmentations $X_{\bullet} \longrightarrow Y$ and $Z_{\bullet} \longrightarrow Y$. The morphism $\varphi$ induces a morphism of complexes

$$
\varphi^{-1}: R f_{*} \mathbb{Q}_{X} \longrightarrow R g_{*} \mathbb{Q}_{Z}
$$

by using the Godment resolutions as we mentioned in 4.1. The cone of the morphism $\varphi^{-1}$ is denoted by $C\left(\varphi^{-1}\right)$. Then $H^{i}\left(C\left(\varphi^{-1}\right)\right)$ underlies a graded polarizable variation of $\mathbb{Q}$-mixed Hodge structure for every $i$.

Proof. A filtration $L$ on $C\left(\varphi^{-1}\right)$ is defined by

$$
L_{m} C\left(\varphi^{-1}\right)^{n}=L_{m-1}\left(R f_{*} \mathbb{Q}_{x_{\bullet}}\right)^{n+1} \oplus L_{m}\left(R g_{*} \mathbb{Q}_{z_{\bullet}}\right)^{n}
$$

where $L$ on the right hand sides denotes the filtrations defined in the proof of Lemma 4.5.

The morphism $\varphi: Z_{\bullet} \longrightarrow X_{\bullet}$ induces another morphisms of complexes

$$
\varphi^{*}: R f_{*} \Omega_{X \bullet / Y} \longrightarrow R g_{*} \Omega_{Z / Y}
$$

which makes the diagram of complexes

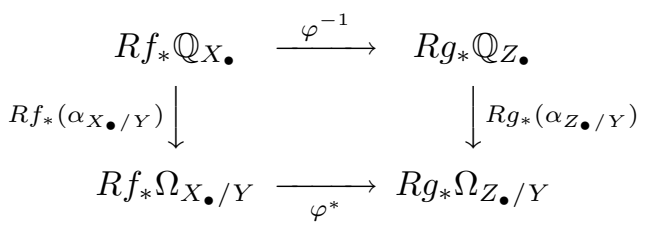

commutes, where $\alpha_{X \bullet / Y}$ and $\alpha_{Z / Y}$ are the canonical morphism as in the proof of Lemma 4.5. Now we consider the mixed cone of the morphism $\varphi^{*}$ (see e.g. [PS, $3.4])$, that is, the cone $C\left(\varphi^{*}\right)$ equipped with the filtrations

$$
\begin{aligned}
& L_{m} C\left(\varphi^{*}\right)^{n}=L_{m-1}\left(R f_{*} \Omega_{X \bullet / Y}\right)^{n+1} \oplus L_{m}\left(R g_{*} \Omega_{Z \bullet} / Y\right)^{n} \\
& F^{p} C\left(\varphi^{*}\right)^{n}=F^{p}\left(R f_{*} \Omega_{X_{\bullet} / Y}\right)^{n+1} \oplus F^{p}\left(R g_{*} \Omega_{Z_{\bullet} / Y}\right)^{n}
\end{aligned}
$$


where $L$ and $F$ on the right hand sides denote the filtrations defined in the proof of Lemma 4.5. Then the commutative diagram (4.8.1) induces a morphism of filtered complexes $\alpha:\left(C\left(\varphi^{-1}\right), L\right) \longrightarrow\left(C\left(\varphi^{*}\right), L\right)$ which induces a filtered quasiisomorphism $\left(C\left(\varphi^{-1}\right), L\right) \otimes \mathcal{O}_{Y} \longrightarrow\left(C\left(\varphi^{*}\right), L\right)$. Then we have

$$
\begin{aligned}
\operatorname{Gr}_{m}^{L} C\left(\varphi^{-1}\right) & =\mathrm{Gr}_{m-1}^{L} R f_{*} \mathbb{Q}_{X}[1] \oplus \mathrm{Gr}_{m}^{L} R g_{*} \mathbb{Q}_{Z} \cdot \\
& =R\left(f_{-m+1}\right)_{*} \mathbb{Q}_{X_{-m}}[m] \oplus R\left(g_{-m}\right)_{*} \mathbb{Q}_{Z_{-m}}[m]
\end{aligned}
$$

and

$$
\begin{aligned}
\left(\mathrm{Gr}_{m}^{L}\right. & \left.C\left(\varphi^{*}\right), F\right) \\
& =\left(\mathrm{Gr}_{m-1}^{L} R f_{*} \Omega_{X \bullet / Y}[1], F\right) \oplus\left(\mathrm{Gr}_{m}^{L} R g_{*} \Omega_{Z_{\bullet} / Y}, F\right) \\
& =\left(R\left(f_{-m+1}\right)_{*} \Omega_{X_{-m+1} / Y}[m], F\right) \oplus\left(R\left(g_{-m}\right)_{*} \Omega_{Z_{-m} / Y}[m], F\right)
\end{aligned}
$$

for every $m$. Therefore the data

$$
\left(E_{1}^{p, q}\left(C\left(\varphi^{-1}\right), L\right),\left(E_{1}^{p, q}\left(C\left(\varphi^{*}\right), L\right), F_{\text {rec }}\right), E_{1}^{p, q}(\alpha)\right)
$$

is a polarizable variation of $\mathbb{Q}$-Hodge structure of weight $q$. Then the same argument in the proof of Lemma 4.5 implies that the spectral sequences $E_{r}^{p, q}\left(C\left(\varphi^{-1}\right), L\right)$ and $E_{r}^{p, q}\left(C\left(\varphi^{*}\right), L\right)$ degenerate at $E_{2}$-terms, the spectral sequence $E_{r}^{p, q}\left(C\left(\varphi^{*}\right), F\right)$ degenerates at $E_{1}$-terms and that the data

$$
\left(\left(H^{i}\left(C\left(\varphi^{-1}\right)\right), L[i]\right),\left(H^{i}\left(C\left(\varphi^{*}\right)\right), L[i], F\right), H^{i}(\alpha)\right)
$$

is a graded polarizable pre-variation of $\mathbb{Q}$-mixed Hodge structure on $Y$ for every $i$.

What remains to prove is the Griffiths transversality. We consider the complexes $\Omega_{X \bullet}, \Omega_{Z_{\bullet}}, g_{\bullet}^{-1} \Omega_{Y}$ and $f_{\bullet}^{-1} \Omega_{Y}$ with the decreasing filtration $G$ as in the proof of Lemma 4.5. We have the commutative diagram

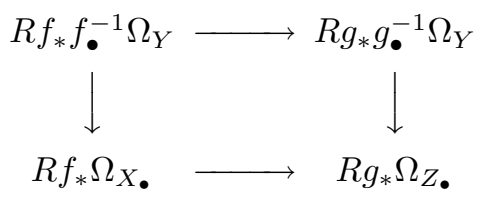

where the vertical arrows are filtered quasi-isomorphism with respect to the filtration $G$. The top horizontal arrow is denoted by $\psi^{-1}$ and the bottom by $\psi^{*}$ for a while. Considering the cones $C\left(\psi^{-1}\right)$ and $C\left(\psi^{*}\right)$ with the filtration $G$ defined by the same way as $F$ in (4.8.2), we obtain a commutative diagram of quasi-isomorphisms

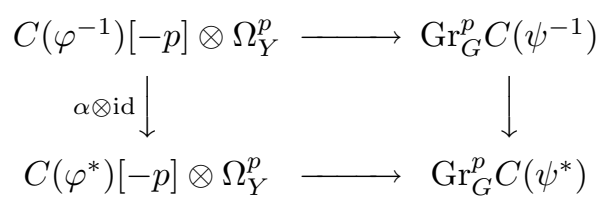


for every $p$. Then we can check the Griffiths transversality by the same way as in the proof of Lemma 4.5.

4.9. Now we review Steenbrink's results in [St1], [St2] and fix the notation for the later use.

Let $X$ be a smooth complex variety and $f: X \longrightarrow \Delta$ a projective surjective morphism. We set $X^{*}=f^{-1}\left(\Delta^{*}\right), E=f^{-1}(0)$. The coordinate function on $\Delta$ is denoted by $t$. We assume that $E_{\text {red }}$ is a simple normal crossing divisor on $X$ and $f: X^{*} \longrightarrow \Delta^{*}$ is a smooth morphism. Moreover, we assume that $R^{i} f_{*} \mathbb{Q}_{X^{*}}$ are of unipotent monodromy for all $i$ for simplicity.

A finite decreasing filtration $G$ on $\Omega_{X}(\log E)$ is defined by

$$
\begin{aligned}
& G^{0} \Omega_{X}(\log E)=\Omega_{X}(\log E) \\
& G^{1} \Omega_{X}(\log E)=\operatorname{Im}\left(f^{-1} \Omega_{\Delta}^{1}(\log 0) \otimes \Omega_{X}(\log E)[-1] \longrightarrow \Omega_{X}(\log E)\right) \\
& G^{2} \Omega_{X}(\log E)=0
\end{aligned}
$$

as in (4.3.1). Then the morphism

$$
\nabla: R^{i} f_{*} \Omega_{X / \Delta}(\log E) \longrightarrow \Omega_{\Delta}^{1}(\log 0) \otimes R^{i} f_{*} \Omega_{X / \Delta}(\log E)
$$

is obtained as the morphism of $E_{1}$-terms of the spectral sequence

$$
E_{r}^{p, q}\left(R f_{*} \Omega_{X / \Delta}(\log E), G\right) .
$$

The restriction $\left.\nabla\right|_{\Delta^{*}}$ is identified with $d \otimes$ id on $\mathcal{O}_{\Delta^{*}} \otimes R^{i} f_{*} \mathbb{Q}_{X^{*}}$ via the isomorphisms

$$
\left.R^{i} f_{*} \Omega_{X / \Delta}(\log E)\right|_{\Delta^{*}} \simeq R^{i} f_{*} \Omega_{X^{*} / \Delta^{*}} \simeq \mathcal{O}_{\Delta^{*}} \otimes R^{i} f_{*} \mathbb{Q}_{X^{*}}
$$

by definition.

Steenbrink proved that $R^{i} f_{*} \Omega_{X / \Delta}(\log E)$ is a locally free coherent $\mathcal{O}_{\Delta}$-module and $\operatorname{Res}_{0}(\nabla)$ is nilpotent. Therefore $R^{i} f_{*} \Omega_{X / \Delta}(\log E)$ is the canonical extension of $\mathcal{O}_{\Delta^{*}} \otimes R^{i} f_{*} \mathbb{Q}_{X^{*}}$ for every $i$. Once we know the local freeness of $R^{i} f_{*} \Omega_{X / \Delta}(\log E)$, the canonical morphism

$$
R^{i} f_{*} \Omega_{X / \Delta}(\log E) \otimes \mathbb{C}(0) \stackrel{\simeq}{\longrightarrow} H^{i}\left(E, \Omega_{X / \Delta}(\log E) \otimes \mathcal{O}_{E}\right)
$$

is an isomorphism for every $i$.

The filtration $G$ on $\Omega_{X}(\log E)$ induces a filtration on $\Omega_{X}(\log E) \otimes \mathcal{O}_{E}$, which is denoted by $G$ again. Then we have

$$
\operatorname{Gr}_{G}^{1} \Omega_{X}(\log E) \otimes \mathcal{O}_{E} \simeq\left(\Omega_{X / \Delta}(\log E) \otimes \mathcal{O}_{E}\right)[-1]
$$

because we have the identification

$$
G^{1} \Omega_{X}(\log E)=d \log t \wedge \Omega_{X}(\log E)[-1] \simeq \Omega_{X / \Delta}(\log E)[-1]
$$


where $d \log t=d t / t$. Therefore we have

$$
\begin{aligned}
& E_{1}^{0, i}\left(R \Gamma\left(E, \Omega_{X}(\log E) \otimes \mathcal{O}_{E}\right), G\right) \simeq H^{i}\left(E, \Omega_{X / \Delta}(\log E) \otimes \mathcal{O}_{E}\right) \\
& E_{1}^{1, i}\left(R \Gamma\left(E, \Omega_{X}(\log E) \otimes \mathcal{O}_{E}\right), G\right) \simeq H^{i}\left(E, \Omega_{X / \Delta}(\log E) \otimes \mathcal{O}_{E}\right)
\end{aligned}
$$

for every $i$. Then the morphism of $E_{1}$-terms

$$
H^{i}\left(E, \Omega_{X / \Delta}(\log E) \otimes \mathcal{O}_{E}\right) \longrightarrow H^{i}\left(E, \Omega_{X / \Delta}(\log E) \otimes \mathcal{O}_{E}\right)
$$

coincides with $\operatorname{Res}_{0}(\nabla)$ under the identification (4.9.1).

In [St1], Steenbrink constructed a cohomological $\mathbb{Q}$-mixed Hodge complex, denoted by

$$
A_{X / \Delta}=\left(\left(A_{X / \Delta}^{\mathbb{Q}}, W\right),\left(A_{X / \Delta}^{\mathbb{C}}, W, F\right), \alpha_{X / \Delta}\right)
$$

in this article, which admits a filtered quasi-isomorphism

$$
\theta_{X / \Delta}:\left(\Omega_{X / \Delta}(\log E) \otimes \mathcal{O}_{E_{\text {red }}}, F\right) \longrightarrow\left(A_{X / \Delta}^{\mathbb{C}}, F\right)
$$

where the filtration $F$ on the left hand side denotes the filtration induced by the stupid filtration on $\Omega_{X / \Delta}(\log E)$. Because the canonical morphism

$$
H^{i}\left(E, \Omega_{X / \Delta}(\log E) \otimes \mathcal{O}_{E}\right) \longrightarrow H^{i}\left(E_{\mathrm{red}}, \Omega_{X / \Delta}(\log E) \otimes \mathcal{O}_{E_{\mathrm{red}}}\right)
$$

is an isomorphism for every $i$ by the unipotent monodromy condition, we have the isomorphisms

$$
\begin{aligned}
R^{i} f_{*} \Omega_{X / \Delta}(\log E) \otimes \mathbb{C}(0) & \stackrel{\simeq}{\longrightarrow} H^{i}\left(E, \Omega_{X / \Delta}(\log E) \otimes \mathcal{O}_{E}\right) \\
& \simeq H^{i}\left(E_{\mathrm{red}}, \Omega_{X / \Delta}(\log E) \otimes \mathcal{O}_{E_{\mathrm{red}}}\right) \\
& \stackrel{\simeq}{\longrightarrow} H^{i}\left(E_{\mathrm{red}}, A_{X / \Delta}^{\mathbb{C}}\right)
\end{aligned}
$$

for every $i$.

Here we just recall the definition of $A_{X / \Delta}^{\mathbb{C}}$ in [St1]. The filtration $W_{X}(E)$ denotes the increasing filtration on $\Omega_{X}(\log E)$ defined by the order of poles along $E$ as usual. The complex $A_{X / \Delta}^{\mathbb{C}}$ is the single complex associated to the double complex $\left(\left(A_{X / \Delta}^{\mathbb{C}}\right)^{p, q}, d^{\prime}, d^{\prime \prime}\right)$ given by

$$
\left(A_{X / \Delta}^{\mathbb{C}}\right)^{p, q}=\Omega_{X}^{p+q+1}(\log E) / W_{X}(E)_{q}
$$

with the differentials

$$
\begin{aligned}
& d^{\prime}=-d:\left(A_{X / \Delta}^{\mathbb{C}}\right)^{p, q} \longrightarrow\left(A_{X / \Delta}^{\mathbb{C}}\right)^{p+1, q} \\
& d^{\prime \prime}=-d \log t \wedge:\left(A_{X / \Delta}^{\mathbb{C}}\right)^{p, q} \longrightarrow\left(A_{X / \Delta}^{\mathbb{C}}\right)^{p, q+1}
\end{aligned}
$$

where $d$ is the morphism induced from the differential of $\Omega_{X}(\log E)$ and where $d \log t \wedge$ denotes the morphism given by the wedge product with $d \log t=d t / t$. For 
the definitions of $W$ and $F$ on $A_{X / \Delta}^{\mathbb{C}}$, see [St1, 4.17]. The morphism given by

$$
\Omega_{X}^{p}(\log E) \ni \omega \mapsto d \log t \wedge \omega \in\left(A_{X / \Delta}^{\mathbb{C}}\right)^{p, 0}
$$

induces the morphism (4.9.3)

$$
\theta_{X / \Delta}: \Omega_{X / \Delta}(\log E) \otimes \mathcal{O}_{E_{\text {red }}} \longrightarrow A_{X / \Delta}^{\mathbb{C}}
$$

which turns out to be a filtered quasi-isomorphism with respect to the filtrations $F$ on the both sides (see [St1, Lemma (4.15)]). The composite

$$
\Omega_{X}(\log E) \otimes \mathcal{O}_{E} \longrightarrow \Omega_{X}(\log E) \otimes \mathcal{O}_{E_{\mathrm{red}}} \stackrel{\theta_{X / \Delta}}{\longrightarrow} A_{X / \Delta}^{\mathbb{C}}
$$

is also denoted by $\theta_{X / \Delta}$ by abuse of the notation.

On the other hand, the projection

$$
\Omega_{X}^{p}(\log E) \longrightarrow \Omega_{X}^{p}(\log E) / W_{X}(E)_{0}=\left(A_{X / \Delta}^{\mathbb{C}}\right)^{p-1,0} \subset\left(A_{X / \Delta}^{\mathbb{C}}[-1]\right)^{p}
$$

induces the morphism

$$
\pi_{X / \Delta}: \Omega_{X}^{p}(\log E) \otimes \mathcal{O}_{E} \longrightarrow\left(A_{X / \Delta}^{\mathbb{C}}[-1]\right)^{p}
$$

for every $p$. Moreover, the projection

$$
\begin{aligned}
\left(A_{X / \Delta}^{\mathbb{C}}\right)^{p, q}=\Omega_{X}^{p+q+1}(\log D) / & W_{X}(E)_{q} \\
& \longrightarrow \Omega_{X}^{p+q+1}(\log E) / W_{X}(E)_{q+1}=\left(A_{X / \Delta}^{\mathbb{C}}\right)^{p-1, q+1}
\end{aligned}
$$

induces a morphism of bifiltered complexes

$$
\left(A_{X / \Delta}^{\mathbb{C}}, W, F\right) \longrightarrow\left(A_{X / \Delta}^{\mathbb{C}}, W[-2], F[-1]\right)
$$

denoted by $\nu_{X / \Delta}$ (see $[$ St1, (4.22), Proposition (4.23)]). Then we have

$$
d \pi_{X / \Delta}=\pi_{X / \Delta} d+\nu_{X / \Delta} \theta_{X / \Delta}: \Omega_{X}^{p}(\log E) \otimes \mathcal{O}_{E} \longrightarrow\left(A_{X / \Delta}^{\mathbb{C}}\right)^{p}
$$

for every $p$, where $d$ on the left hand side is the differential of $A_{X / \Delta}^{\mathbb{C}}[-1]$.

We set

$$
N_{X / \Delta}=H^{i}\left(\nu_{X / \Delta}\right): H^{i}\left(E_{\text {red }}, A_{X / \Delta}^{\mathbb{C}}\right) \longrightarrow H^{i}\left(E_{\text {red }}, A_{X / \Delta}^{\mathbb{C}}\right)
$$

for every $i$. It is proved that the morphism

$$
N_{X / \Delta}^{k}: \operatorname{Gr}_{k}^{W} H^{i}\left(E_{\text {red }}, A_{X / \Delta}^{\mathbb{C}}\right) \longrightarrow \operatorname{Gr}_{-k}^{W} H^{i}\left(E_{\text {red }}, A_{X / \Delta}^{\mathbb{C}}\right)
$$

is an isomorphism for every $k \geq 0$ (see Steenbrink [St1], El Zein [E1], Saito [Sa], Guillen-Navarro Aznar [GN], Usui [U]). 
The complex $B_{X / \Delta}$ is defined by

$$
B_{X / \Delta}^{p}=\left(A_{X / \Delta}^{\mathbb{C}}\right)^{p-1} \oplus\left(A_{X / \Delta}^{\mathbb{C}}\right)^{p}
$$

with the differential

$$
d(x, y)=\left(-d x-\nu_{X / \Delta}(y), d y\right)
$$

for $x \in\left(A_{X / \Delta}^{\mathbb{C}}\right)^{p-1}$ and $y \in\left(A_{X / \Delta}^{\mathbb{C}}\right)^{p}$, where $d$ denotes the differential of the complex $A_{X / \Delta}^{\mathbb{C}}$. We define a filtration $G$ on $B_{X / \Delta}$ by

$$
\begin{aligned}
G^{0} B_{X / \Delta} & =B_{X / \Delta} \\
G^{1} B_{X / \Delta} & =A_{X / \Delta}^{\mathbb{C}}[-1] \\
G^{2} B_{X / \Delta} & =0
\end{aligned}
$$

where $A_{X / \Delta}^{\mathbb{C}}[-1]$ is regarded as a subcomplex of $B_{X / \Delta}$ by the inclusion $\left(A_{X / \Delta}^{\mathbb{C}}\right)^{p-1} \longrightarrow$ $B_{X / \Delta}^{p}$ for every $p$.

A morphism

$$
\Omega_{X}^{p}(\log E) \otimes \mathcal{O}_{E} \ni \omega \mapsto\left(\pi_{X / \Delta}(\omega), \theta_{X / \Delta}(\omega)\right) \in\left(A_{X / \Delta}^{\mathbb{C}}\right)^{p-1} \oplus\left(A_{X / \Delta}^{\mathbb{C}}\right)^{p}
$$

defines a morphism of complexes

$$
\eta_{X / \Delta}: \Omega_{X}(\log E) \otimes \mathcal{O}_{E} \longrightarrow B_{X / \Delta}
$$

by (4.9.5). It is easy to check that the morphism $\eta_{X / \Delta}$ preserves the filtration $G$ on the both sides. Note that the diagrams

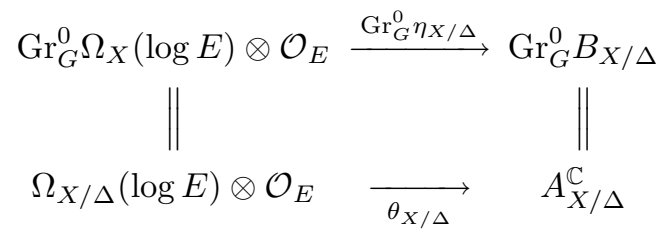

and

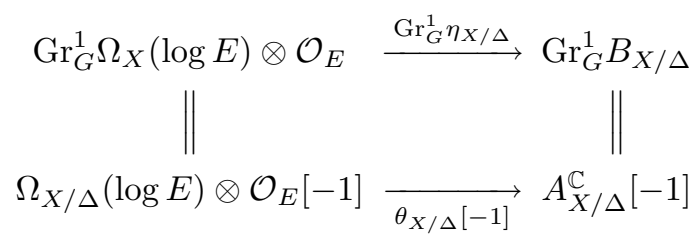

are commutative. Considering the morphisms between $E_{1}$-terms induced by $\eta_{X / \Delta}$, we have

$$
\operatorname{Res}_{0}(\nabla)=-N_{X / \Delta}
$$

on $H^{i}\left(E_{\mathrm{red}}, A_{X / \Delta}^{\mathbb{C}}\right)$ under the isomorphism (4.9.4). 
We remark that the construction above is functorial. For the rational structure $A_{X / \Delta}^{\mathbb{Q}}$, we can use the construction by Steenbrink-Zucker [SZ].

In [St2], the local freeness of $R^{i} f_{*} \Omega_{X / \Delta}^{p}(\log E)$ is proved.

Lemma 4.10. Let $f: X_{\bullet} \longrightarrow Y$ be a projective surjective augmented strict semisimplicial variety to a smooth algebraic variety $Y$. Moreover, assume that $X_{p}$ is smooth for every $p$. Then there exists a Zariski open dense subset $Y^{*}$ of $Y$ such that $\left.\left(R^{i} f_{*} \mathbb{Q}_{X_{\bullet}}\right)\right|_{Y^{*}}$ underlies an admissible graded polarizable variation of $\mathbb{Q}$-mixed Hodge structure for every $i$.

Proof. There exists a non-empty Zariski open subset $Y^{*}$ of $Y$ such that the morphism $f: X_{\bullet} \longrightarrow Y$ is smooth over $Y^{*}$. We set $X_{\bullet}^{*}=f^{-1}\left(Y^{*}\right)$ and denote the induced morphisms $X_{\bullet}^{*} \longrightarrow Y^{*}$ by the same letter $f$ by abuse of the language. Then $\left.\left(R^{i} f_{*} \mathbb{Q}_{X}\right)\right|_{Y^{*}}$ underlies a graded polarizable variation of $\mathbb{Q}$-mixed Hodge structure by Lemma 4.5 .

It is sufficient to prove that the graded polarizable variation of $\mathbb{Q}$-mixed Hodge structure above is admissible. Because the graded polarizable variation of $\mathbb{Q}$-mixed Hodge structure constructed in Lemma 4.5 commutes with the base change as in Remark 4.7, we may assume $Y=\Delta$ and $Y^{*}=\Delta^{*}$.

Our variation of graded polarizable $\mathbb{Q}$-mixed Hodge structure has a $\mathbb{Z}$-structure. Therefore, the quasi-unipotency of the monodromy around the origin is obvious by Remark 3.13. Thus we have the property (3.11.1) in Definition 3.11 (i). Once we know the quasi-unipotency of the monodromy, Lemma 1.9.1 in [Ks] allows us to assume that $f: X_{\bullet} \longrightarrow \Delta$ is of unipotent monodromy. Moreover we may assume that $f^{-1}(0)_{\text {red }}$ is a simple normal crossing divisor on the smooth semi-simplicial complex variety $X_{\bullet}$. We set $E_{\bullet}=f_{\bullet}^{-1}(0)$. Note that $E_{\bullet}$ and $E_{\bullet \text { red }}=\left\{\left(E_{p}\right)_{\text {red }}\right\}_{p \geq 0}$ are strict semi-simplicial subvarieties of $X_{\bullet}$.

Consider the bifiltered complex

$$
\left(R f_{*} \Omega_{X_{\bullet} / \Delta}\left(\log E_{\bullet}\right), L, F\right)
$$

where $L$ is defined in (4.2.1) and $F$ as in (4.2.3). We trivially have

$$
\left.\left(R f_{*} \Omega_{X_{\bullet} / \Delta}\left(\log E_{\bullet}\right), L, F\right)\right|_{\Delta^{*}}=\left(R f_{*} \Omega_{X_{\bullet}^{*} / \Delta^{*}}, L, F\right)
$$

by definition.

Step 1. In this first step, we prove the local freeness of several coherent $\mathcal{O}_{\Delta^{-}}$ modules.

Consider the spectral sequence

$$
\left(E_{r}^{p, q}\left(R f_{*} \Omega_{X \cdot / \Delta}\left(\log E_{\bullet}\right), L\right), F_{\text {rec }}\right)
$$


associated to the filtration $L$ on the complex $R f_{*} \Omega_{X_{\bullet} / \Delta}\left(\log E_{\bullet}\right)$.

By (4.2.2), we have

$$
E_{1}^{p, q}\left(R f_{*} \Omega_{X_{\bullet} / \Delta}\left(\log E_{\bullet}\right), L\right) \simeq R^{q} f_{p *} \Omega_{X_{p} / \Delta}\left(\log E_{p}\right)
$$

which is the canonical extension of

$$
\begin{aligned}
E_{1}^{p, q}\left(R f_{*} \Omega_{X_{\bullet}^{*} / \Delta^{*}}, L\right) & \simeq R^{q} f_{p *} \Omega_{X_{p}^{*} / \Delta^{*}} \\
& \simeq \mathcal{O}_{\Delta^{*}} \otimes R^{q} f_{p *} \mathbb{Q}_{X_{p}^{*}} \simeq \mathcal{O}_{\Delta^{*}} \otimes E_{1}^{p, q}\left(R f_{*} \mathbb{Q}_{X_{\bullet}^{*}}, L\right)
\end{aligned}
$$

by [St1]. Because taking the canonical extension is an exact functor by [D1, Proposition $5.2(\mathrm{~d})], E_{2}^{p, q}\left(R f_{*} \Omega_{X_{\bullet} / \Delta}\left(\log E_{\bullet}\right), L\right)$ is the canonical extension of

$$
E_{2}^{p, q}\left(R f_{*} \Omega_{X_{\bullet}^{*} / \Delta^{*}}, L\right) \simeq \mathcal{O}_{\Delta^{*}} \otimes_{\mathbb{Q}} E_{2}^{p, q}\left(R f_{*} \mathbb{Q}_{X_{\bullet}^{*}}, L\right) .
$$

Therefore $E_{2}^{p, q}\left(R f_{*} \Omega_{X \bullet / \Delta}\left(\log E_{\bullet}\right), L\right)$ is a locally free coherent $\mathcal{O}_{\Delta}$-module. Once we know the local freeness of $E_{2}$-terms, the spectral sequence

$$
E_{r}^{p, q}\left(R f_{*} \Omega_{X_{\bullet} / \Delta}\left(\log E_{\bullet}\right), L\right)
$$

degenerates at $E_{2}$-terms because its restriction on $\Delta^{*}$ degenerates at $E_{2}$-terms. Thus we obtain that

$$
\begin{aligned}
E_{2}^{p, q}\left(R f_{*} \Omega_{X_{\bullet} / \Delta}\left(\log E_{\bullet}\right), L\right) & \simeq E_{\infty}^{p, q}\left(R f_{*} \Omega_{X_{\bullet} / \Delta}\left(\log E_{\bullet}\right), L\right) \\
& \simeq \operatorname{Gr}_{-p}^{L} R^{p+q} f_{*} \Omega_{X_{\bullet} / \Delta}\left(\log E_{\bullet}\right)
\end{aligned}
$$

is locally free for every $p, q$. In particular, $R^{i} f_{*} \Omega_{X_{\bullet} / \Delta}\left(\log E_{\bullet}\right)$ is a locally free coherent $\mathcal{O}_{\Delta}$-module with the property

$$
\left.R^{i} f_{*} \Omega_{X_{\bullet} / \Delta}\left(\log E_{\bullet}\right)\right|_{\Delta^{*}} \simeq R^{i} f_{*} \Omega_{X_{\bullet} / \Delta} \simeq \mathcal{O}_{\Delta^{*}} \otimes R^{i} f_{*} \mathbb{Q}_{X_{\bullet}}
$$

for every $i$.

The morphism of $E_{1}$-terms

$$
d_{1}: R^{q} f_{p *} \Omega_{X_{p} / \Delta}\left(\log E_{p}\right) \longrightarrow R^{q} f_{p+1 *} \Omega_{X_{p+1} / \Delta}\left(\log E_{p+1}\right)
$$

preserves the filtration $F$ because $F_{\text {rec }}=F_{d}=F_{d^{*}}$ on the $E_{1}$-terms in general and because $F_{\text {rec }}$ on $E_{1}^{p, q}\left(R f_{*} \Omega_{X_{\bullet} / \Delta}\left(\log E_{\bullet}\right), L\right)$ coincides with $F$ on $R^{q} f_{p *} \Omega_{X_{p} / \Delta}\left(\log E_{p}\right)$ under the isomorphism (4.10.1). On the other hand, the filtration $F$ on $R^{q} f_{p *} \Omega_{X_{p} / \Delta}\left(\log E_{p}\right)$ coincides with the filtration obtained by the nilpotent orbit theorem in $[\mathrm{Sc}]$ because

$$
\mathrm{Gr}_{F}^{r} R^{q} f_{p *} \Omega_{X_{p} / \Delta}\left(\log E_{p}\right) \simeq R^{q-r} f_{p *} \Omega_{X_{p} / \Delta}^{r}\left(\log E_{p}\right)
$$

is locally free for every $r$ (see Corollary 5.2 below). By the $S L_{2}$-orbit theorem in $[\mathrm{Sc}]$,

$$
\left(R^{q} f_{p *} \Omega_{X_{p} / \Delta}\left(\log E_{p}\right) \otimes \mathbb{C}(0), W, F\right)
$$


underlies a $\mathbb{Q}$-mixed Hodge structure for every $p, q$, where $W$ denotes the monodromy weight filtration. On the other hand, the morphism

$$
d_{1}(0): R^{q} f_{p *} \Omega_{X_{p} / \Delta}\left(\log E_{p}\right) \otimes \mathbb{C}(0) \longrightarrow R^{q} f_{p+1 *} \Omega_{X_{p+1} / \Delta}\left(\log E_{p+1}\right) \otimes \mathbb{C}(0)
$$

induced by the morphism $d_{1}$ in (4.10.2) underlies a morphism of $\mathbb{Q}$-mixed Hodge structures because the restriction of $d_{1}$ on $\Delta^{*}$ preserves the $\mathbb{Q}$-structures $R^{q} f_{p *} \mathbb{Q} X_{p}^{*}$ and $R^{q} f_{p+1 *} \mathbb{Q}_{X_{p+1}^{*}}$. Therefore $d_{1}(0)$ is strictly compatible with the filtrations $F$ on $R^{q} f_{p *} \Omega_{X_{p} / \Delta}\left(\log E_{p}\right) \otimes \mathbb{C}(0)$ and $R^{q} f_{p+1 *} \Omega_{X_{p+1} / \Delta}\left(\log E_{p+1}\right) \otimes \mathbb{C}(0)$. In other words, the morphism

$$
\begin{aligned}
d_{1}(0): E_{1}^{p, q}\left(R f_{*} \Omega_{X_{\bullet} / \Delta}\right. & \left.\left(\log E_{\bullet}\right), L\right) \otimes \mathbb{C}(0) \\
& \longrightarrow E_{1}^{p+1, q}\left(R f_{*} \Omega_{X_{\bullet} / \Delta}\left(\log E_{\bullet}\right), L\right) \otimes \mathbb{C}(0)
\end{aligned}
$$

is strictly compatible with the filtrations $F_{\text {rec }}$ on the both sides.

Applying (iv) in Lemma 3.4 to the complex

$$
\left(E_{1}^{\bullet, q}\left(R f_{*} \Omega_{X_{\bullet} / \Delta}\left(\log E_{\bullet}\right), L\right), F_{\text {rec }}\right)
$$

we conclude that

$$
\operatorname{Gr}_{F_{\mathrm{rec}}}^{r} E_{2}^{p, q}\left(R f_{*} \Omega_{X_{\bullet} / \Delta}\left(\log E_{\bullet}\right), L\right)
$$

is locally free coherent $\mathcal{O}_{\Delta}$-module for every $p, q, r$ and that $F_{\text {rec }}=F_{d}=F_{d^{*}}$ on $E_{2}^{p, q}\left(R f_{*} \Omega_{X_{\bullet} / \Delta}\left(\log E_{\bullet}\right), L\right)$. Therefore

$$
\operatorname{Gr}_{F_{\mathrm{rec}}}^{r} E_{2}^{p, q}\left(R f_{*} \Omega_{X \bullet / \Delta}\left(\log E_{\bullet}\right), L\right) \simeq \operatorname{Gr}_{F}^{r} \operatorname{Gr}_{-p}^{L} R^{p+q} f_{*} \Omega_{X_{\bullet} / \Delta}\left(\log E_{\bullet}\right)
$$

is locally free for every $p, q, r$. Moreover, the spectral sequence

$$
E_{r}^{p, q}\left(R f_{*} \Omega_{X_{\bullet} / \Delta}\left(\log E_{\bullet}\right), F\right)
$$

degenerates at $E_{1}$-terms by the lemma on two filtrations as before.

Step 2. The canonical morphism

$$
\Omega_{X_{\bullet} / \Delta}\left(\log E_{\bullet}\right) \longrightarrow \Omega_{X_{\bullet} / \Delta}\left(\log E_{\bullet}\right) \otimes \mathcal{O}_{E_{\bullet}}
$$

induces the morphism of complexes

$$
R f_{*} \Omega_{X_{\bullet} / \Delta}\left(\log E_{\bullet}\right) \otimes \mathbb{C}(0) \longrightarrow R \Gamma\left(E_{\bullet}, \Omega_{X_{\bullet} / \Delta}\left(\log E_{\bullet}\right) \otimes \mathcal{O}_{E_{\bullet}}\right)
$$

preserving the filtration $L$ on the both sides. Then the morphism of the spectral sequences induces the morphism

$$
\begin{aligned}
E_{r}^{p, q}\left(R f_{*} \Omega_{X_{\bullet} / \Delta}\right. & \left.\left(\log E_{\bullet}\right), L\right) \otimes \mathbb{C}(0) \\
& \longrightarrow E_{r}^{p, q}\left(R \Gamma\left(E_{\bullet}, \Omega_{X_{\bullet} / \Delta}\left(\log E_{\bullet}\right) \otimes \mathcal{O}_{E_{\bullet}}\right), L\right)
\end{aligned}
$$


for every $p, q, r$. For the case of $r=1$, the morphism above coincides with the canonical morphism

$$
R^{q} f_{p *} \Omega_{X_{p} / \Delta}\left(\log E_{p}\right) \otimes \mathbb{C}(0) \longrightarrow H^{q}\left(E_{p}, \Omega_{X_{p} / \Delta}\left(\log E_{p}\right) \otimes \mathcal{O}_{E_{p}}\right)
$$

which is an isomorphism for every $p, q$ as mentioned in 4.9. Therefore the morphism

$$
\begin{aligned}
H^{p}\left(E _ { 1 } ^ { \bullet , q } \left(R f_{*} \Omega_{X_{\bullet} / \Delta}\right.\right. & \left.\left.\left(\log E_{\bullet}\right), L\right) \otimes \mathbb{C}(0)\right) \\
& \longrightarrow E_{2}^{p, q}\left(R \Gamma\left(E_{\bullet}, \Omega_{X_{\bullet} / \Delta}\left(\log E_{\bullet}\right) \otimes \mathcal{O}_{E_{\bullet}}\right), L\right)
\end{aligned}
$$

is an isomorphism for every $p, q$. Moreover, the canonical morphism

$$
\begin{aligned}
E_{2}^{p, q}\left(R f_{*} \Omega_{X_{\bullet} / \Delta}\right. & \left.\left(\log E_{\bullet}\right), L\right) \otimes \mathbb{C}(0) \\
& \longrightarrow H^{p}\left(E_{1}^{\bullet, q}\left(R f_{*} \Omega_{X_{\bullet} / \Delta}\left(\log E_{\bullet}\right), L\right) \otimes \mathbb{C}(0)\right)
\end{aligned}
$$

is an isomorphism for every $p, q$, because $E_{2}^{p, q}\left(R f_{*} \Omega_{X_{\bullet} / \Delta}\left(\log E_{\bullet}\right), L\right)$ is locally free for every $p, q$ as proved in Step 1. Thus we know that the morphism (4.10.4) is an isomorphism for every $p, q$ and for $r=2$. Therefore the $E_{2}$-degeneracy of $E_{r}^{p, q}\left(R f_{*} \Omega_{X_{\bullet} / \Delta}\left(\log E_{\bullet}\right), L\right)$ implies the $E_{2}$-degeneracy of

$$
E_{r}^{p, q}\left(R \Gamma\left(E_{\bullet}, \Omega_{X_{\bullet} / \Delta}\left(\log E_{\bullet}\right) \otimes \mathcal{O}_{E_{\bullet}}\right), L\right) .
$$

Moreover, we have the canonical isomorphism

$$
\mathrm{Gr}_{m}^{L} R^{i} f_{*} \Omega_{X_{\bullet} / \Delta}\left(\log E_{\bullet}\right) \otimes \mathbb{C}(0) \longrightarrow \operatorname{Gr}_{m}^{L} H^{i}\left(E_{\bullet}, \Omega_{X_{\bullet} / \Delta}\left(\log E_{\bullet}\right) \otimes \mathcal{O}_{E_{\bullet}}\right)
$$

for every $i, m$. In particular, the canonical morphism

$$
R^{i} f_{*} \Omega_{X \bullet / \Delta}\left(\log E_{\bullet}\right) \otimes \mathbb{C}(0) \longrightarrow H^{i}\left(E_{\bullet}, \Omega_{X \bullet / \Delta}\left(\log E_{\bullet}\right) \otimes \mathcal{O}_{E_{\bullet}}\right)
$$

is an isomorphism for every $i$.

Step 3. Considering the filtered complex

$$
\left(R f_{*} \Omega_{X_{\bullet}}\left(\log E_{\bullet}\right), G\right)
$$

we obtain the log integrable connection

$$
\nabla: R^{i} f_{*} \Omega_{X_{\bullet} / \Delta}\left(\log E_{\bullet}\right) \longrightarrow \Omega_{\Delta}^{1}(\log 0) \otimes R^{i} f_{*} \Omega_{X_{\bullet} / \Delta}\left(\log E_{\bullet}\right)
$$

as the morphism of $E_{1}$-terms of the spectral sequence. It is clear that $\left.\nabla\right|_{\Delta^{*}}$ coincides with the connection (4.5.6).

On the other hand, we consider

$$
\left(R \Gamma\left(E_{\bullet}, \Omega_{X_{\bullet}}\left(\log E_{\bullet}\right) \otimes \mathcal{O}_{E_{\bullet}}\right), G\right)
$$


with the identification

$$
G^{1} \Omega_{X \bullet}\left(\log E_{\bullet}\right) \otimes \mathcal{O}_{E_{\bullet}} \simeq\left(\Omega_{X_{\bullet} / \Delta}\left(\log E_{\bullet}\right) \otimes \mathcal{O}_{E_{\bullet}}\right)[-1]
$$

as in (4.9.2). The same procedure in 4.9 shows the fact that the morphism of $E_{1}$-terms

$$
\begin{aligned}
H^{i}\left(E_{\bullet}, \Omega_{X_{\bullet} / \Delta}\right. & \left.\left(\log E_{\bullet}\right) \otimes \mathcal{O}_{E_{\bullet}}\right) \\
& \longrightarrow H^{i}\left(E_{\bullet}, \Omega_{X_{\bullet} / \Delta}\left(\log E_{\bullet}\right) \otimes \mathcal{O}_{E_{\bullet}}\right)
\end{aligned}
$$

coincides with $\operatorname{Res}_{0}(\nabla)$ via the isomorphism (4.10.5).

Step 4. The data $A_{X_{\bullet} / \Delta}$ gives us an object on the semi-simplicial variety $E_{\bullet}$ red because Steenbrink's construction in 4.9 is functorial as mentioned there. Then the data

$$
\left(R \Gamma\left(E_{\bullet \text { red }}, A_{X \bullet / \Delta}\right), L, \delta(W, L), F\right)
$$

is obtained. We set

$$
A_{\mathbb{C}}=R \Gamma\left(E_{\bullet \text { red }}, A_{X_{\bullet} / \Delta}^{\mathbb{C}}\right)
$$

for simplicity. The morphism

$$
\theta_{X_{\bullet} / \Delta}: \Omega_{X_{\bullet} / \Delta}\left(\log E_{\bullet}\right) \otimes \mathcal{O}_{E_{\bullet}} \longrightarrow A_{X_{\bullet} / \Delta}^{\mathbb{C}}
$$

induces the morphism

$$
\theta: R \Gamma\left(E_{\bullet}, \Omega_{X_{\bullet} / \Delta}\left(\log E_{\bullet}\right) \otimes \mathcal{O}_{E_{\bullet}}\right) \longrightarrow A_{\mathbb{C}}
$$

which preserves the filtrations $L$ and $F$. Because

$$
H^{i}\left(\mathrm{Gr}_{m}^{L} \theta\right): H^{i}\left(\mathrm{Gr}_{m}^{L} R \Gamma\left(E_{\bullet}, \Omega_{X_{\bullet} / \Delta}\left(\log E_{\bullet}\right) \otimes \mathcal{O}_{E_{\bullet}}\right)\right) \longrightarrow H^{i}\left(\operatorname{Gr}_{m}^{L} A_{\mathbb{C}}\right)
$$

coincides with the isomorphism

$$
\left.H^{i}\left(E_{-m}, \Omega_{X_{-m} / \Delta}\left(\log E_{-m}\right) \otimes \mathcal{O}_{E_{-m}}\right)\right) \longrightarrow H^{i}\left(\left(E_{-m}\right)_{\text {red }}, A_{X_{-m} / \Delta}^{\mathbb{C}}\right)
$$

in (4.9.4) for every $i, m$, the morphism $\theta$ is a filtered quasi-isomorphism with respect to $L$. In particular, $\theta$ is a quasi-isomorphism, that is,

$$
H^{i}(\theta): H^{i}\left(E_{\bullet}, \Omega_{X \bullet / \Delta}\left(\log E_{\bullet}\right) \otimes \mathcal{O}_{E_{\bullet}}\right) \longrightarrow H^{i}\left(A_{\mathbb{C}}\right)
$$

is an isomorphism for every $i$.

Moreover, the morphism

$$
\nu_{X_{\bullet} / \Delta}:\left(A_{X_{\bullet} / \Delta}^{\mathbb{C}}, W, F\right) \longrightarrow\left(A_{X \bullet / \Delta}^{\mathbb{C}}, W[-2], F[-1]\right)
$$


induces the morphism

$$
\left(A_{\mathbb{C}}, L, \delta(W, L), F\right) \longrightarrow\left(A_{\mathbb{C}}, L, \delta(W, L)[-2], F[-1]\right)
$$

which we simply denote by $\nu$. Because of the property

$$
\nu\left(\delta(W, L)_{m} A_{\mathbb{C}}\right) \subset \delta(W, L)_{m-2} A_{\mathbb{C}}
$$

$\nu$ is a nilpotent endomorphism. We set

$$
N=H^{i}(\nu): H^{i}\left(A_{\mathbb{C}}\right) \longrightarrow H^{i}\left(A_{\mathbb{C}}\right)
$$

for every $i$.

On the other hand, we obtain

$$
\left(R \Gamma\left(E_{\bullet \text { red }}, B_{X_{\bullet} / \Delta}\right), G\right)
$$

from $B_{X \bullet / \Delta}$ with the filtration $G$. By definition, we have

$$
R \Gamma\left(E_{\bullet \text { red }}, B_{X_{\bullet} / \Delta}\right)^{n}=A_{\mathbb{C}}^{n-1} \oplus A_{\mathbb{C}}^{n}
$$

and

$$
d(x, y)=(-d x-\nu(y), d y)
$$

for $x \in A_{\mathbb{C}}^{n-1}$ and $y \in A_{\mathbb{C}}^{n}$, where $d$ is the differential of the complex $A_{\mathbb{C}}$. Moreover, the filtration $G$ on $R \Gamma\left(E_{\bullet \text { red }}, B_{X_{\bullet} / \Delta}\right)$ satisfies

$$
G^{1} R \Gamma\left(E_{\bullet \text { red }}, B_{X_{\bullet} / \Delta}\right)=A_{\mathbb{C}}[-1]
$$

as in 4.9. The morphism

$$
\eta_{X_{\bullet} / \Delta}: \Omega_{X_{\bullet}}\left(\log E_{\bullet}\right) \otimes \mathcal{O}_{E_{\bullet}} \longrightarrow B_{X_{\bullet} / \Delta}
$$

induced by (4.9.7) gives us the morphism

$$
\eta: R \Gamma\left(E_{\bullet}, \Omega_{X_{\bullet}}\left(\log E_{\bullet}\right) \otimes \mathcal{O}_{E_{\bullet}}\right) \longrightarrow R \Gamma\left(E_{\bullet \text { red }}, B_{X_{\bullet} / \Delta}\right)
$$

preserving the filtration $G$. Note that the diagrams

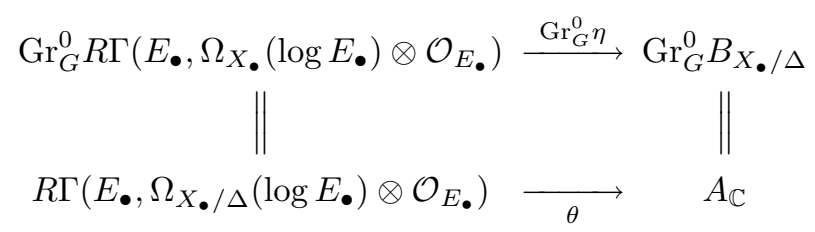

and

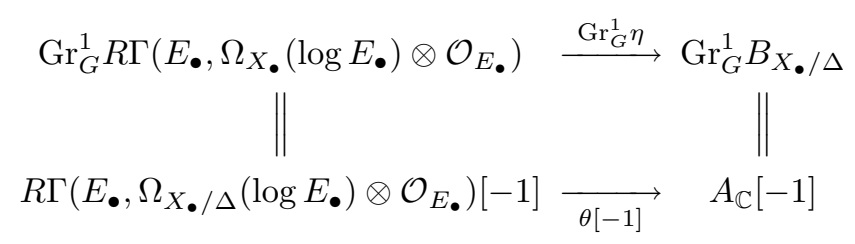


are commutative by definition. Thus the morphism of $E_{1}$-terms (4.10.6) coincides with $-N$ under the isomorphism (4.10.9). Therefore $\operatorname{Res}_{0}(\nabla)$ is identified with $-N$ via the isomorphisms (4.10.5) and (4.10.9). Because $\nu$ is nilpotent, the morphism $N$ is nilpotent, and then so is $\operatorname{Res}_{0}(\nabla)$. Thus we conclude that $R^{i} f_{*} \Omega_{X \bullet / \Delta}\left(\log E_{\bullet}\right)$ is the canonical extension of $R^{i} f_{*} \Omega_{X_{\bullet}^{*} / \Delta^{*}} \simeq \mathcal{O}_{\Delta^{*}} \otimes R^{i} f_{*} \mathbb{Q}_{X_{\bullet}^{*}}$.

Step 5. We can easily see that the data (4.10.7) is a $\mathbb{Q}$-mixed Hodge complex filtered by $L$ (for the definition of filtered $\mathbb{Q}$-mixed Hodge complex, see e.g. [E2, 6.1.4 Définition]). Moreover, the spectral sequence associated to the filtration $L$ degenerates at $E_{2}$-terms because $\theta$ in (4.10.8) is a filtered quasi-isomorphism and because the spectral sequence for $\left(R \Gamma\left(E_{\bullet}, \Omega_{X_{\bullet} / \Delta}\left(\log E_{\bullet}\right) \otimes \mathcal{O}_{E_{\bullet}}\right), L\right)$ degenerates at $E_{2}$-terms. The filtration induced by $\delta(W, L)$ on

$$
\mathrm{Gr}_{m}^{L} A_{\mathbb{C}} \simeq R \Gamma\left(\left(E_{-m}\right)_{\mathrm{red}}, A_{X_{-m} / \Delta}^{\mathbb{C}}\right)
$$

coincides with $W[m]$ and the morphism $\operatorname{Gr}_{m}^{L} \nu$ coincides with $\nu_{X_{-m} / \Delta}$ for every $m$. Hence the filtration $\delta(W, L)[-m]$ on $H^{i}\left(\mathrm{Gr}_{m}^{L} A_{\mathbb{C}}\right)$ is the monodromy weight filtration by the isomorphism (4.9.6). Therefore $\delta(W, L)$ on $H^{i}\left(A_{\mathbb{C}}\right)$ is the monodromy weight filtration of $N=H^{i}(\nu)$ relative to the filtration $L$ by Lemma 3.17.

Thus the condition (3.11.2) is obtained by the local freeness in Step 1 and by the fact that $R^{i} f_{\bullet *} \Omega_{X \bullet / \Delta}\left(\log E_{\bullet}\right)$ is the canonical extension of $R^{i} f_{\bullet *} \Omega_{X_{\bullet}^{*} / \Delta^{*}} \simeq$ $\mathcal{O}_{\Delta^{*}} \otimes R^{i} f_{\bullet *} \mathbb{Q}_{X_{\bullet}^{*}}$ for every $i$ in Step 3. Moreover, the condition (3.11.3) is proved by the existence of the monodromy weight filtration of $N$ relative to $L$ in Step 5 and by the fact that $N$ coincides with $-\operatorname{Res}_{0}(\nabla)$ in Step 3 .

Remark 4.11. Let $(V, W)$ be a finite dimensional $\mathbb{Q}$-vector space equipped with a finite increasing filtration $W$ and $N$ a nilpotent endomorphism of $V$ preserving the filtration $W$. On the $\mathbb{C}$-vector space $V_{\mathbb{C}}=\mathbb{C} \otimes V$, the filtration $W$ and the nilpotent endomorphism $N_{\mathbb{C}}=\mathrm{id} \otimes N$ are induced by the trivial way. Then the existence of the monodromy weight filtration of $N$ relative to $W$ on $V$ is equivalent to the existence of the monodromy weight filtration of $N_{\mathbb{C}}$ relative to $W$ on $V_{\mathbb{C}}$. We can check this equivalence by using Theorem (2.20) in [SZ].

Lemma 4.12 (GPVMHS for relative cohomology). Let $f: X_{\bullet} \longrightarrow Y$ and $g$ : $Z_{\bullet} \longrightarrow Y$ be projective augmented strict semi-simplicial varieties and $\varphi: Z_{\bullet} \longrightarrow$ $X_{\bullet}$ a morphism of semi-simplicial varieties compatible with the augmentations $X_{\bullet} \longrightarrow Y$ and $Z_{\bullet} \longrightarrow Y$. The cone of the canonical morphism $\varphi^{-1}: R f_{*} \mathbb{Q}_{X} \bullet \longrightarrow$ $R g_{*} \mathbb{Q}_{Z_{\bullet}}$ is denoted by $C\left(\varphi^{-1}\right)$ as in Lemma 4.8. Take the open subset $Y^{*}$ such that $f: X_{\bullet} \longrightarrow Y$ and $g: Z_{\bullet} \longrightarrow Y$ are smooth over $Y^{*}$. Then $\left.H^{i}\left(C\left(\varphi^{-1}\right)\right)\right|_{Y^{*}}$ underlies an admissible graded polarizable variation of $\mathbb{Q}$-mixed Hodge structure for every $i$. 
Proof. By Lemma 4.8, $\left.H^{i}\left(C\left(\varphi^{-1}\right)\right)\right|_{Y^{*}}$ is a graded polarizable variation of $\mathbb{Q}$-mixed Hodge structure. We will prove the admissibility of it.

As in Lemma 4.10, we may assume the following:

- $Y=\Delta, Y^{*}=\Delta^{*}$.

- $f_{p}: X_{p} \longrightarrow \Delta$ and $g_{q}: Z_{q} \longrightarrow \Delta$ are of unipotent monodromy for all $p, q$.

- $f^{-1}(0)_{\text {red }}$ and $g^{-1}(0)_{\text {red }}$ are simple normal crossing divisors on $X$ • and $Z$ • respectively.

We set $E_{\bullet}=f^{-1}(0)$ and $F_{\bullet}=g^{-1}(0)$. The morphism $\varphi: Z_{\bullet} \longrightarrow X_{\bullet}$ induces the morphism of complexes

$$
\varphi^{*}: R f_{*} \Omega_{X_{\bullet} / \Delta}\left(\log E_{\bullet}\right) \longrightarrow R g_{*} \Omega_{Z_{\bullet} / \Delta}\left(\log F_{\bullet}\right)
$$

as in the proof of Lemma 4.8. Then we consider $C\left(\varphi^{*}\right)$ equipped with filtrations $L$ and $F$ defined by the same way as (4.8.2) from the filtrations $L$ and $F$ on the complexes $R f_{*} \Omega_{X_{\bullet} / \Delta}\left(\log E_{\bullet}\right)$ and $R g_{*} \Omega_{Z_{\bullet} / \Delta}\left(\log F_{\bullet}\right)$. Then $\left.C\left(\varphi^{*}\right)\right|_{\Delta^{*}}$ induces the mixed Hodge structure on $H^{i}\left(C\left(\varphi^{-1}\right)\right)$ as in the proof of Lemma 4.8.

Because we have

$$
\begin{aligned}
& \left(E_{1}^{p, q}\left(C\left(\varphi^{*}\right), L\right), F\right) \\
& \quad=\left(R^{q}\left(f_{p+1}\right)_{*} \Omega_{X_{p+1} / \Delta}\left(\log E_{p}\right), F\right) \oplus\left(R^{q}\left(g_{p}\right)_{*} \Omega_{Z_{p} / \Delta}\left(\log F_{p}\right), F\right)
\end{aligned}
$$

as in the proof of Lemma 4.8, the same argument as Step 1 of the proof of Lemma 4.10 shows that the spectral sequence $E_{r}^{p, q}\left(C\left(\varphi^{*}\right), L\right)$ degenerates at $E_{2}$-terms and that

$$
\operatorname{Gr}_{F_{\text {rec }}}^{r} E_{2}^{p, q}\left(C\left(\varphi^{*}\right), L\right) \simeq \operatorname{Gr}_{F}^{r} \operatorname{Gr}_{-p}^{L} H^{p+q}\left(C\left(\varphi^{*}\right)\right)
$$

are locally free coherent $\mathcal{O}_{\Delta}$-modules for all $p, q, r$. Moreover, the spectral sequence $E_{r}^{p, q}\left(C\left(\varphi^{*}\right), F\right)$ degenerates at $E_{1}$-terms by the lemma on two filtrations as usual.

Let $A_{X_{\bullet} / \Delta}^{\mathbb{C}}$ and $A_{Z_{\bullet} / \Delta}^{\mathbb{C}}$ be the complexes defined in Step 4 of the proof of Lemma 4.10. The morphism $\varphi$ induces a morphism of trifiltered complexes

$$
\begin{aligned}
\left(R \Gamma\left(E_{\bullet \text { red }}, A_{X_{\bullet} / \Delta}^{\mathbb{C}}\right), L, \delta(W, L), F\right) \\
\longrightarrow\left(R \Gamma\left(F_{\bullet \text { red }}, A_{Z_{\bullet} / \Delta}^{\mathbb{C}}\right), L, \delta(W, L), F\right)
\end{aligned}
$$

by using the Godment resolution as in 4.1. This morphism of complexes is denoted by $\psi$ for a while. On the complex $C(\psi)$, the filtrations $L$ and $F$ are defined by the same way as in (4.8.2) and the filtration $\delta(W, L)$ by the same way as $L$. We can easily check that $(C(\psi), L, \delta(W, L), F)$ underlies a filtered $\mathbb{Q}$-mixed Hodge complex. The composites of the morphisms (4.10.3) and (4.10.8) for $X_{\bullet}$ and $Z_{\bullet}$ fit 
in the commutative diagram

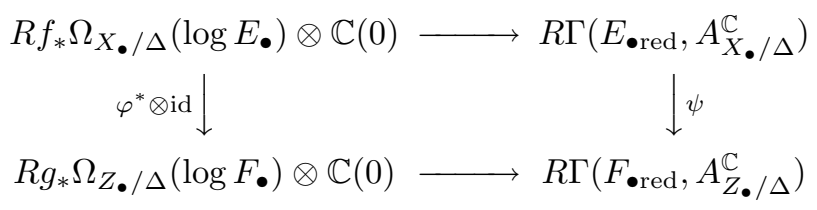

from which the morphism of complexes $C\left(\varphi^{*}\right) \otimes \mathbb{C}(0) \longrightarrow C(\psi)$ preserving the filtrations $L$ and $F$ is obtained. This morphism induces an isomorphism

$$
E_{1}^{p, q}\left(C\left(\varphi^{*}\right), L\right) \otimes \mathbb{C}(0) \longrightarrow E_{1}^{p, q}(C(\psi), L)
$$

because the morphism between $E_{1}$-terms coincides with the direct sum of the isomorphisms (4.10.5) for $X_{\bullet}$ and $Z_{\bullet}$. Then the canonical morphism

$$
E_{2}^{p, q}\left(C\left(\varphi^{*}\right), L\right) \otimes \mathbb{C}(0) \longrightarrow E_{2}^{p, q}(C(\psi), L)
$$

is an isomorphism because the local freeness of $E_{2}^{p, q}\left(C\left(\varphi^{*}\right), L\right)$ implies that the canonical morphism

$$
E_{2}^{p, q}\left(C\left(\varphi^{*}\right), L\right) \otimes \mathbb{C}(0) \longrightarrow H^{p}\left(E_{1}^{\bullet, q}\left(C\left(\varphi^{*}\right), L\right) \otimes \mathbb{C}(0)\right)
$$

is an isomorphism. Therefore the spectral sequence $E_{r}^{p, q}(C(\psi), L)$ degenerates at $E_{2}$-terms and the canonical morphism

$$
H^{i}\left(C\left(\varphi^{*}\right)\right) \otimes \mathbb{C}(0) \longrightarrow H^{i}(C(\psi))
$$

is an isomorphism for every $i$, under which the filtration $L$ on the both sides coincides.

The morphisms (4.10.10) for $X_{\bullet}$ and $Z_{\bullet}$ induce the morphism of complexes

$$
(C(\psi), L, \delta(W, L), F) \longrightarrow(C(\psi), L, \delta(W, L)[-2], F[-1])
$$

which is denoted by $\nu$ again.

By using the mapping cone of the canonical morphism

$$
\left(R f_{*} \Omega_{X \bullet}\left(\log E_{\bullet}\right), G\right) \longrightarrow\left(R g_{*} \Omega_{Z}\left(\log F_{\bullet}\right), G\right)
$$

with the decreasing filtrations $G$ defined by the same way as $F$ in (4.8.2), we obtain the log integrable connection $\nabla$ on $H^{i}\left(C\left(\varphi^{*}\right)\right)$ for every $i$ by the same way as in the proof of Lemma 4.8. By definition, the restriction of this $\nabla$ on $\Delta^{*}$ coincides with the original $\nabla$ on $\left.H^{i}\left(C\left(\varphi^{*}\right)\right)\right|_{\Delta^{*}}$. Similarly, the morphism $\varphi: Z_{\bullet} \longrightarrow X \bullet$ induces a 
morphism of filtered complexes $\left(B_{X_{\bullet} / \Delta}, G\right) \longrightarrow\left(B_{Z_{\bullet} / \Delta}, G\right)$ such that the diagram

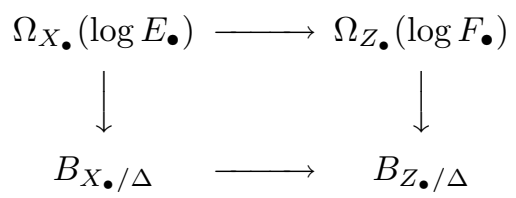

is commutative. By considering the cone of the morphism

$$
R \Gamma\left(E_{\bullet \text { red }}, B_{X_{\bullet} / \Delta}\right) \longrightarrow R \Gamma\left(F_{\bullet \text { red }}, B_{Z_{\bullet} / \Delta}\right)
$$

with the decreasing filtration $G$, the residue $\operatorname{Res}_{0}(\nabla)$ on $H^{i}\left(C\left(\varphi^{*}\right)\right) \otimes \mathbb{C}(0)$ is identified with $-H^{i}(\nu)$ for every $i$. Because the morphism $\nu$ is trivially nilpotent, we conclude that $\operatorname{Res}_{0}(\nabla)$ is nilpotent. Therefore $H^{i}\left(C\left(\varphi^{*}\right)\right)$ is the canonical extension of $\left.H^{i}\left(C\left(\varphi^{*}\right)\right)\right|_{\Delta^{*}}$. Thus the condition (3.11.2) is satisfied by $H^{i}\left(C\left(\varphi^{*}\right)\right)$ for every $i$. Moreover, we can easily see that the filtration $\delta(W, L)[-m]$ on $H^{i}\left(\operatorname{Gr}_{m}^{L} C(\psi)\right)$ is the monodromy weight filtration of $\mathrm{Gr}_{m}^{L} H^{i}(\nu)$ for every $i, m$. Therefore Lemma 3.17 implies that the filtration $\delta(W, L)$ is the monodromy weight filtration of $H^{i}(\nu)$ relative to $L$ on $H^{i}(C(\psi)) \simeq H^{i}\left(C\left(\varphi^{*}\right)\right) \otimes \mathbb{C}(0)$.

Theorem 4.13 (GPVMHS for cohomology with compact support). Let $f: X \longrightarrow$ $Y$ be a projective surjective morphism from a complex variety $X$ onto a smooth complex variety $Y$ and $Z$ a closed subset of $X$. Then there exists a Zariski open dense subset $Y^{*}$ of $Y$ such that $\left.\left(R^{i}\left(\left.f\right|_{X \backslash Z}\right) ! \mathbb{Q}_{X \backslash Z}\right)\right|_{Y^{*}}$ underlies an admissible graded polarizable variation of $\mathbb{Q}$-mixed Hodge structure for every $i$.

Proof. The open immersion $X \backslash Z \longrightarrow X$ and the closed immersion $Z \longrightarrow X$ are denoted by $\iota$ and $j$ respectively. We set $g=f j: Z \longrightarrow Y$. Take cubical hyperresolutions $\varepsilon_{Z}: Z_{\bullet} \longrightarrow Z$ and $\varepsilon_{X}: X_{\bullet} \longrightarrow X$ which fits in the commutative diagram

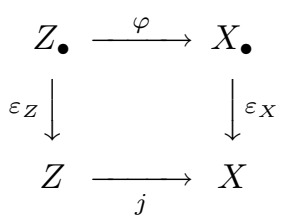

for some morphism $\varphi: Z_{\bullet} \longrightarrow X_{\bullet}$ of cubical varieties. The cone of the canonical morphism $\varphi^{-1}: R\left(f \varepsilon_{X}\right)_{*} \mathbb{Q}_{X} \bullet \longrightarrow R\left(g \varepsilon_{Z}\right)_{*} \mathbb{Q}_{Z}$. is denoted by $C\left(\varphi^{-1}\right)$ as in Lemma 4.12. Then the composite of the canonical morphisms

$$
R\left(\left.f\right|_{X \backslash Z}\right)_{!} \mathbb{Q}_{X \backslash Z} \simeq R f_{*} \iota_{!} \mathbb{Q}_{X \backslash Z} \longrightarrow R f_{*} \mathbb{Q}_{X} \longrightarrow R\left(f \varepsilon_{X}\right)_{*} \mathbb{Q}_{X}
$$

induces the quasi-isomorphism $R\left(\left.f\right|_{X \backslash Z}\right) ! \mathbb{Q}_{X \backslash Z} \longrightarrow C\left(\varphi^{-1}\right)[-1]$ from which we obtain the conclusion by considering the filtration $L[-1]$ on $C\left(\varphi^{-1}\right)[-1]$. 
4.14. Let $(X, D)$ be a simple normal crossing pair with $D$ reduced. The irreducible decompositions of $X$ and $D$ are given by

$$
X=\bigcup_{i \in I} X_{i}, \quad D=\bigcup_{\lambda \in \Lambda} D_{\lambda}
$$

respectively. Fixing orders $<$ on $\Lambda$ and $I$, we set

$$
D_{k} \cap X_{l}=\coprod_{\substack{\lambda_{0}<\lambda_{1}<\cdots<\lambda_{k} \\ i_{0}<i_{1}<\cdots<i_{l}}} D_{\lambda_{0}} \cap D_{\lambda_{1}} \cap \cdots \cap D_{\lambda_{k}} \cap X_{i_{0}} \cap X_{i_{1}} \cap \cdots \cap X_{i_{l}}
$$

for $k, l \geq 0$. Here we use the convention

$$
\begin{aligned}
& D_{k}=D_{k} \cap X_{-1}=\coprod_{\lambda_{0}<\lambda_{1}<\cdots<\lambda_{k}} D_{\lambda_{0}} \cap D_{\lambda_{1}} \cap \cdots \cap D_{\lambda_{k}} \\
& X_{l}=D_{-1} \cap X_{l}=\coprod_{i_{0}<i_{1}<\cdots<i_{l}} X_{i_{0}} \cap X_{i_{1}} \cap \cdots \cap X_{i_{l}}
\end{aligned}
$$

for $k, l \geq 0$. Moreover, we set

$$
(D \cap X)_{n}=\coprod_{k+l+1=n} D_{k} \cap X_{l}
$$

for $n \geq 0$. Thus we obtain projective augmented strict semi-simplicial varieties $\varepsilon_{X}:(D \cap X) \bullet \longrightarrow X, \varepsilon_{D}: D \bullet \longrightarrow D$ and the morphism of semi-simplicial varieties $\varphi: D_{\bullet} \longrightarrow(D \cap X)$ • compatible with the augmentation $\varepsilon_{X}$ and $\varepsilon_{D}$. We remark that $D_{k} \cap X_{l}$ are smooth for all $k, l$ by the definition of simple normal crossing pair. Therefore $\varepsilon_{D}: D \bullet \longrightarrow D$ is a hyperresolution of $D$. Now we will see that $\varepsilon_{X}:(D \cap X) \bullet \longrightarrow X$ is also a hyperresolution of $X$. It is sufficient to prove that $\varepsilon_{X}$ is of cohomological descent. The cone of the canonical morphism

$$
\mathbb{Q}_{X} \longrightarrow\left(\varepsilon_{X}\right)_{*} \mathbb{Q}_{(D \cap X) \bullet}=R\left(\varepsilon_{X}\right)_{*} \mathbb{Q}_{(D \cap X)} \bullet
$$


is the single complex associated to the double complex

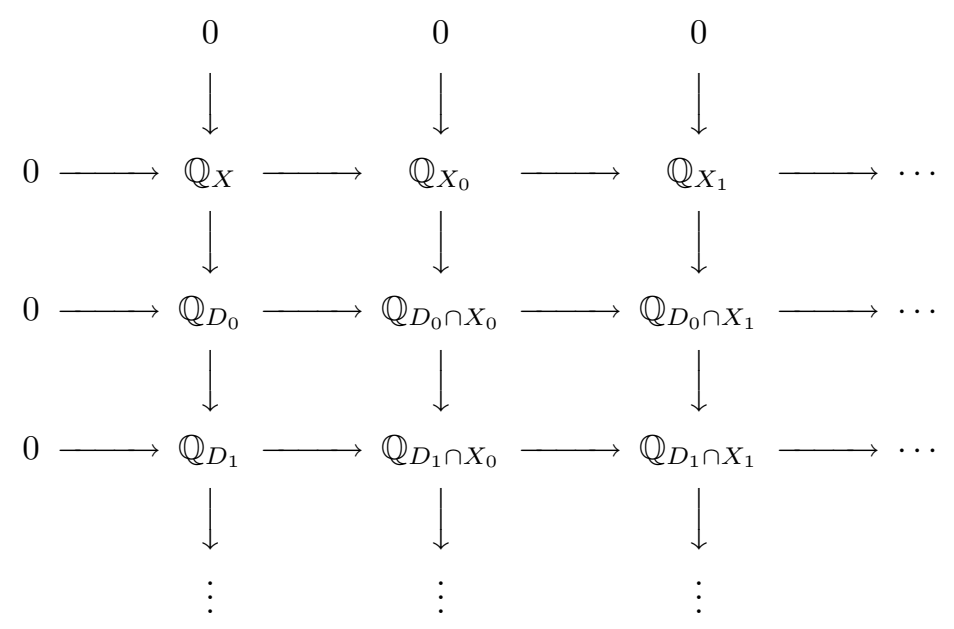

shifted by 1 . All the lines of the diagram above are exact because they are the Mayer-Vietoris exact sequences for $X$ and for $D_{k}$. Then the single complex associated to the double complex above is acyclic. Thus we obtain that the canonical morphism $\mathbb{Q}_{X} \longrightarrow\left(\varepsilon_{X}\right)_{*} \mathbb{Q}_{(D \cap X)}$. is a quasi-isomorphism.

Theorem 4.15 (GPVMHS for snc pair). Let $(X, D)$ be a simple normal crossing pair with $D$ reduced and $f: X \longrightarrow Y$ a projective surjective morphism to a smooth algebraic variety $Y$. Let $Y^{*}$ be the non-empty Zariski open subset of $Y$ such that all the strata of $(X, D)$ are smooth over $Y^{*}$. Then $\left.\left(R^{i}\left(\left.f\right|_{X \backslash D}\right) ! \mathbb{Q}_{X \backslash D}\right)\right|_{Y^{*}}$ underlies an admissible graded polarizable variation of $\mathbb{Q}$-mixed Hodge structure for every $i$.

Proof. As we mentioned in 4.14, we have the commutative diagram

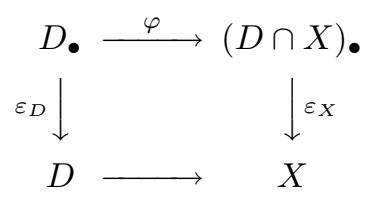

such that $\varepsilon_{D}$ and $\varepsilon_{X}$ are hyperresolutions. Then we obtain the conclusion by the same way as the proof of Theorem 4.13 from Lemma 4.12 .

Remark 4.16. In the situation above, the inverse images of the open subset $Y^{*}$ are indicated by the superscript ${ }^{*}$ such as $X^{*}=f^{-1}\left(Y^{*}\right)$. From the proof of Lemma 4.8, we can check that $\operatorname{Gr}_{F}^{p}\left(\left.\mathcal{O}_{Y^{*}} \otimes\left(R^{i}\left(\left.f\right|_{X \backslash D}\right) ! \mathbb{Q}_{X \backslash D}\right)\right|_{Y^{*}}\right)$ coincides with 
the $(i-p)$-th direct image of the single complex associated to the double complex

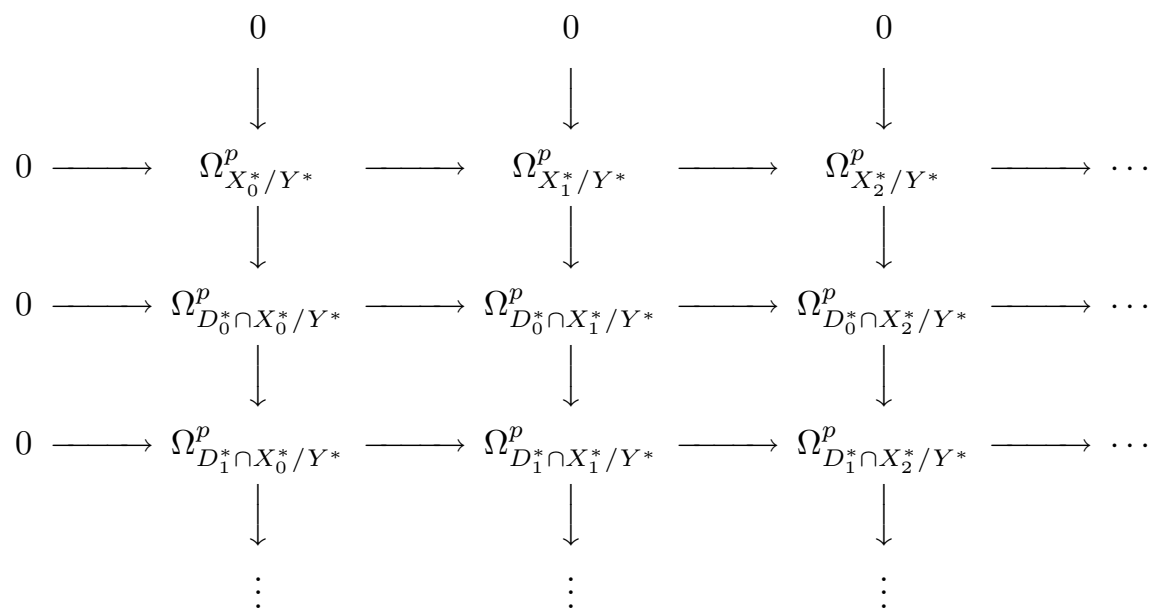

by $f$. Therefore we have the canonical isomorphism

$$
R^{i} f_{*} \mathcal{O}_{X}(-D) \simeq \operatorname{Gr}_{F}^{0}\left(\left.\mathcal{O}_{Y^{*}} \otimes\left(R^{i}\left(\left.f\right|_{X \backslash D}\right) ! \mathbb{Q}_{X \backslash D}\right)\right|_{Y^{*}}\right)
$$

for every $i$.

\section{$\S 5$. Semipositivity theorem}

In this section, we discuss a purely Hodge theoretic aspect of the Fujita-Kawamata semipositivity theorem (cf. [Z] and [Kw1, §4 Semi-positivity]). Our formulation is different from Kawamata's original one but is indispensable for our main theorem: Theorem 7.1 (4). For related topics, see [Mor, Section 5], [F5, Section 5], [F4, 3.2. Semi-positivity theorem], and [Ko5, 8.10]. We use the theory of logarithmic integrable connections. For the basic properties and results on logarithmic integrable connections, see [D1], [Kt], and [Bo, IV. Regular connections, after Deligne] by Bernard Malgrange. For a different approach, see [FFS, Section 4].

We start with easy observations.

Lemma 5.1. Let $X$ be a complex manifold, $U$ a dense open subset of $X$ and $\mathcal{V}$ a locally free $\mathcal{O}_{X}$-module of finite rank. Assume that two $\mathcal{O}_{X}$-submodules $\mathcal{F}$ and $\mathcal{G}$ satisfy the following conditions:

(5.1.1) $\mathcal{G}$ and $\mathcal{V} / \mathcal{G}$ are locally free $\mathcal{O}_{X}$-modules of finite rank.

(5.1.2) $\left.\mathcal{F}\right|_{U}=\left.\mathcal{G}\right|_{U}$

Then we have the inclusion $\mathcal{F} \subset \mathcal{G}$ on $X$. 
Corollary 5.2. Let $X, U$ and $\mathcal{V}$ be as above. Two finite decreasing filtrations $F$ and $G$ on $\mathcal{V}$ satisfy the following conditions:

- $\operatorname{Gr}_{G}^{p} \mathcal{V}$ is a locally free $\mathcal{O}_{X}$-module of finite rank for every $p$.

- $\left.F^{p} \mathcal{V}\right|_{U}=\left.G^{p} \mathcal{V}\right|_{U}$ for every $p$.

Then we have $F^{p} \mathcal{V} \subset G^{p} \mathcal{V}$ on $X$ for every $p$. In particular, $F^{p} \mathcal{V}=G^{p} \mathcal{V}$ for every $p$, if, in addition, $\operatorname{Gr}_{F}^{p} \mathcal{V}$ is locally free of finite rank for every $p$.

5.3. Let $X$ be a complex manifold and $D=\sum_{i \in I} D_{i}$ a simple normal crossing divisor on $X$, where $D_{i}$ is a smooth irreducible divisor on $X$ for every $i \in I$. We set

$$
D(J)=\bigcap_{i \in J} D_{i}, \quad D_{J}=\sum_{i \in J} D_{i}
$$

for any subset $J$. Note that $D(\emptyset)=X$ and $D_{\emptyset}=0$ by definition. Moreover we set $D(J)^{*}=D(J) \backslash D(J) \cap D_{I \backslash J}$ for $J \subset I$. For the case of $J=\emptyset$, we set $X^{*}=D(\emptyset)^{*}=X \backslash D$.

Let $\mathcal{V}$ be a locally free $\mathcal{O}_{X}$-module of finite rank and

$$
\nabla: \mathcal{V} \longrightarrow \Omega_{X}^{1}(\log D) \otimes \mathcal{V}
$$

a logarithmic integrable connection on $\mathcal{V}$. The residue of $\nabla$ along $D_{i}$ is denoted by

$$
\operatorname{Res}_{D_{i}}(\nabla): \mathcal{O}_{D_{i}} \otimes \mathcal{V} \longrightarrow \mathcal{O}_{D_{i}} \otimes \mathcal{V}
$$

We assume the following condition throughout this section:

(5.3.1) $\operatorname{Res}_{D_{i}}(\nabla): \mathcal{O}_{D_{i}} \otimes \mathcal{V} \longrightarrow \mathcal{O}_{D_{i}} \otimes \mathcal{V}$ is nilpotent for every $i \in I$.

This is equivalent to the condition that the local system $\left.\operatorname{Ker}(\nabla)\right|_{X^{*}}$ is of unipotent local monodromy.

5.4. In the situation above, the morphism

$$
\mathrm{id} \otimes \operatorname{Res}_{D_{i}}(\nabla): \mathcal{O}_{D(J)} \otimes \mathcal{V} \longrightarrow \mathcal{O}_{D(J)} \otimes \mathcal{V}
$$

is denoted by $N_{i, D(J)}$ for a subset $J$ of $I$ and for $i \in J$. We simply write $N_{i}$ if there is no danger of confusion. We have

$$
N_{i, D(J)} N_{j, D(J)}=N_{j, D(J)} N_{i, D(J)}
$$

for every $i, j \in J$. For two subsets $J, K$ of $I$ with $K \subset J$, we set $N_{K, D(J)}=$ $\sum_{i \in K} N_{i, D(J)}$, which is nilpotent by the assumption above. Once a subset $J$ is fixed, we use the symbols $N_{K}$ for short. We have the monodromy weight filtration 
$W(K)$ on $\mathcal{O}_{D(J)} \otimes \mathcal{V}$ which is characterized by the condition that $N_{K}^{q}$ induces an isomorphism

$$
\mathrm{Gr}_{q}^{W(K)}\left(\mathcal{O}_{D(J)} \otimes \mathcal{V}\right) \stackrel{\simeq}{\longrightarrow} \operatorname{Gr}_{-q}^{W(K)}\left(\mathcal{O}_{D(J)} \otimes \mathcal{V}\right)
$$

for all $q \geq 0$. For $K=\emptyset, W(\emptyset)$ is trivial, that is, $W(\emptyset)_{-1} \mathcal{O}_{D(J)} \otimes \mathcal{V}=0$ and $W(\emptyset)_{0} \mathcal{O}_{D(J)} \otimes \mathcal{V}=\mathcal{O}_{D(J)} \otimes \mathcal{V}$

For the case of $J=K$, we set

$$
\mathcal{P}_{k}(J)=\operatorname{Ker}\left(N_{J}^{k+1}: \operatorname{Gr}_{k}^{W(J)}\left(\mathcal{O}_{D(J)} \otimes \mathcal{V}\right) \longrightarrow \operatorname{Gr}_{-k-2}^{W(J)}\left(\mathcal{O}_{D(J)} \otimes \mathcal{V}\right)\right)
$$

for every non-negative integer $k$, which is called the primitive part of $\operatorname{Gr}_{k}^{W(J)}\left(\mathcal{O}_{D(J)} \otimes\right.$ $\mathcal{V})$ with respect to $N_{J}$. Then we have the primitive decomposition

$$
\operatorname{Gr}_{k}^{W(J)}\left(\mathcal{O}_{D(J)} \otimes \mathcal{V}\right)=\bigoplus_{l \geq \max (0,-k)} N_{J}^{l}\left(\mathcal{P}_{k+2 l}(J)\right)
$$

for every $k$, and $N_{J}^{l}$ induces an isomorphism

$$
\mathcal{P}_{k+2 l}(J) \longrightarrow N_{J}^{l}\left(\mathcal{P}_{k+2 l}(J)\right)
$$

for every $k, l$ with $\geq \max (0,-k)$.

Lemma 5.5. In the situation above, $\operatorname{Gr}_{k}^{W(K)}\left(\mathcal{O}_{D(J)} \otimes \mathcal{V}\right)$ is a locally free $\mathcal{O}_{D(J)^{-}}$ module of finite rank for every $k$ and for every subsets $J, K$ of $I$ with $K \subset J$.

Proof. Easy by the local description of logarithmic integrable connection (see e.g. Deligne [D1], Katz [Kt]).

Corollary 5.6. In the situation above, we fix a subset $J$ of $I$. For any subset $K$ of $J$ we have the equality

$$
W(K)=W\left(N_{K}(x)\right)
$$

on $\mathcal{V}(x)=\mathcal{V} \otimes \mathbb{C}(x)$ for every point $x \in D(J)$, where the left hand side denotes the filtration on $\mathcal{V}(x)$ induced by $W(K)$.

Remark 5.7. Let $\left(\mathcal{V}_{1}, \nabla_{1}\right)$ and $\left(\mathcal{V}_{2}, \nabla_{2}\right)$ be pairs of locally free sheaves of $\mathcal{O}_{X^{-}}$ modules of finite rank and integrable logarithmic connections on them. We assume that they satisfy the condition in 5.3. If the morphism $\varphi: \mathcal{V}_{1} \longrightarrow \mathcal{V}_{2}$ of $\mathcal{O}_{X^{-}}$ modules is compatible with the connections $\nabla_{1}$ and $\nabla_{2}$, then the diagram

$$
\begin{array}{cr}
\mathcal{O}_{D(J)} \otimes \mathcal{V}_{1} \stackrel{\operatorname{id} \otimes \varphi}{\longrightarrow} \mathcal{O}_{D(J)} \otimes \mathcal{V}_{2} \\
N_{i, D(J)} \downarrow \\
\mathcal{O}_{D(J)} \otimes \mathcal{V}_{1} \underset{\operatorname{id} \otimes \varphi}{\longrightarrow} \mathcal{O}_{D(J)} \otimes \mathcal{V}_{2}
\end{array}
$$


is commutative for every subset $J$ of $I$ and for every $i \in J$. Therefore id $\otimes \varphi$ preserves the filtration $W(K)$ for every $K \subset J$.

5.8. Let $m$ be an integer. For a finite decreasing filtration $F$ on $\mathcal{V}$, we consider the following condition:

$(m \mathrm{MH})$ The triple

$$
(\mathcal{V}(x), W(J)[m], F)
$$

underlies an $\mathbb{R}$-mixed Hodge structure for any subset $J$ of $I$ and for any point $x \in D(J)^{*}$.

Here we remark that we do not assume the local freeness of $\operatorname{Gr}_{F}^{p} \mathcal{V}$ at the beginning.

The following lemma is the counterpart of Schmid's results in [Sc].

Lemma 5.9. Let $U$ be an open subset of $X \backslash D$, such that $X \backslash U$ is nowhere dense analytic subspace of $X$. Moreover, we are given a finite decreasing filtration $F$ on $\left.\mathcal{V}\right|_{U}$. If $\left.(\mathcal{V}, F, \nabla)\right|_{U}$ underlies a polarizable variation of $\mathbb{R}$-Hodge structure of weight $m$ on $U$, then there exists a finite decreasing filtration $\widetilde{F}$ on $\mathcal{V}$ satisfying the following three conditions:

1. $\left.\widetilde{F}^{p} \mathcal{V}\right|_{U}=\left.F^{p} \mathcal{V}\right|_{U}$ for every $p$.

2. $\operatorname{Gr}_{\widetilde{F}}^{p} \mathcal{V}$ is a locally free $\mathcal{O}_{X}$-module of finite rank for every $p$.

3. $\widetilde{F}$ satisfies the condition $(m \mathrm{MH})$ in 5.8 .

Proof. See [Sc].

Lemma 5.10. Let $U$ be as above, and $F$ a finite decreasing filtration on $\mathcal{V}$ in the situation 5.3. We assume that $\left.(\mathcal{V}, F, \nabla)\right|_{U}$ underlies a polarizable variation of $\mathbb{R}$-Hodge structure of weight $m$ on $U$. Then $\operatorname{Gr}_{F}^{p} \mathcal{V}$ is locally free of finite rank for every $p$ if and only if $F$ satisfies the condition $(m \mathrm{MH})$ in 5.8 .

Proof. By the lemma above, there exists a finite decreasing filtration $\widetilde{F}$ on $\mathcal{V}$ satisfying the three conditions above. By Corollary 5.2, the local freeness of $\operatorname{Gr}_{F}^{p} \mathcal{V}$ for every $p$ is equivalent to the equality $F^{p} \mathcal{V}=\widetilde{F}^{p} \mathcal{V}$ for every $p$. If $F=\widetilde{F}$ on $\mathcal{V}, F$ satisfies the condition $(m \mathrm{MH})$ by the lemma above. Thus it suffices to prove the equality $F=\widetilde{F}$ on $\mathcal{V}$ under the assumption that $F$ satisfies the condition $(m \mathrm{MH})$. By Corollary 5.2 again, we have $F^{p} \mathcal{V} \subset \widetilde{F}^{p} \mathcal{V}$ for every $p$. On the other hand, $(\mathcal{V}(x), W(J)[m], F)$ and $(\mathcal{V}(x), W(J)[m], \widetilde{F})$ are $\mathbb{R}$-mixed Hodge structures for every $x \in D(J)^{*}$, if $F$ satisfies the condition $(m \mathrm{MH})$. Therefore we obtain $F(\mathcal{V}(x))=\widetilde{F}(\mathcal{V}(x))$ for every $x \in X$, which implies the equality $F=\widetilde{F}$ on $\mathcal{V}$. 
5.11. In addition to the situation 5.3 , we assume that we are given a finite decreasing filtration $F$ on $\mathcal{V}$ satisfying the following three conditions:

- The Griffiths transversality holds, that is, we have $\nabla\left(F^{p}\right) \subset \Omega_{X}^{1}(\log E) \otimes F^{p-1}$ for every $p$.

- $\left.(\mathcal{V}, F, \nabla)\right|_{X^{*}}$ underlies a polarizable variation of $\mathbb{R}$-Hodge structure of weight $m$.

- $\operatorname{Gr}_{F}^{p} \mathcal{V}$ is locally free of finite rank for every $p$, or equivalently, $F$ satisfies the condition $(m \mathrm{MH})$.

For a subset $J$ of $I$, the Griffiths transversality implies the condition

$$
N_{i}\left(F^{p}\left(\mathcal{O}_{D(J)} \otimes \mathcal{V}\right)\right) \subset F^{p-1}\left(\mathcal{O}_{D(J)} \otimes \mathcal{V}\right)
$$

for every $p$ and for every $i \in J$.

Lemma 5.12. In the situation above, we have

(1) $N_{i}\left(W(K)_{k}\right) \subset W(K)_{k-1}$ for every $i \in K$ and for every $k$,

(2) $W(J)$ is the monodromy weight filtration of $N_{K}$ relative to the filtration $W(J \backslash$ $K)$

on $\mathcal{O}_{D(J)} \otimes \mathcal{V}$ for every two subsets $J, K$ of $I$ with $K \subset J$.

Proof. See Cattani-Kaplan [CK, (3.3) Theorem, $(3,4)]$ and Steenbrink-Zucker [SZ, (3.12) Theorem].

Corollary 5.13. In the situation 5.3 and 5.11, the induced filtration $F$ on

$$
\operatorname{Gr}_{k}^{W(J)}\left(\mathcal{O}_{D(J)} \otimes \mathcal{V}\right)
$$

satisfies the property $((m+k) \mathrm{MH})$ for any subset $J$ of $I$.

Proof. Take a subset $K$ of $I \backslash J$. For any point $x \in D(J \cup K)^{*}$, the triple

$$
(\mathcal{V}(x), W(J \cup K)[m], F)
$$

underlies an $\mathbb{R}$-mixed Hodge structure because $F$ satisfies the condition $(m \mathrm{MH})$ by the assumption. Moreover, the morphism $(2 \pi \sqrt{-1})^{-1} N_{J}(x)$ is a morphism of $\mathbb{R}$-mixed Hodge structures of type $(-1,-1)$ by the condition $(2)$ in the lemma above and by the Griffiths transversality. Therefore

$$
\left(\mathrm{Gr}_{k}^{W(J)} \mathcal{V}(x), W(J \cup K)[m], F\right)
$$

is an $\mathbb{R}$-mixed Hodge structure. On the other hand, we have

$$
W(J \cup K)\left(\operatorname{Gr}_{k}^{W(J)} \mathcal{V}(x)\right)=W(K)\left(\operatorname{Gr}_{k}^{W(J)} \mathcal{V}(x)\right)[k],
$$


by (2) in the lemma above. Thus

$$
\left(\mathrm{Gr}_{k}^{W(J)} \mathcal{V}(x), W(K)[m+k], F\right)
$$

underlies an $\mathbb{R}$-mixed Hodge structure.

5.14. In the situation 5.3 and 5.11 we fix a subset $J$ of $I$. We have an exact sequence

$$
\begin{aligned}
& 0 \longrightarrow \Omega_{D(J)}^{1}\left(\log D(J) \cap D_{I \backslash J}\right) \longrightarrow \Omega_{X}^{1}(\log D) \otimes \mathcal{O}_{D(J)} \\
& \longrightarrow \quad \mathcal{O}_{D(J)}^{\oplus|J|} \quad \longrightarrow 0,
\end{aligned}
$$

where $|J|$ denotes the cardinality of $J$. On the other hand, the integrable log connection $\nabla$ induces a commutative diagram

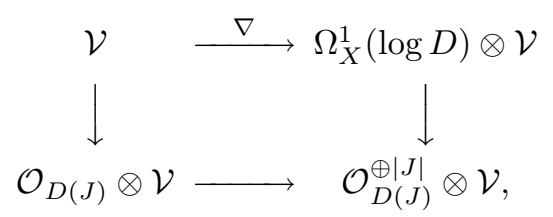

where the bottom horizontal arrow coincides with $\bigoplus_{i \in J} N_{i, D(J)}$ under the identification $\mathcal{O}_{D(J)}^{\oplus|J|} \otimes \mathcal{V} \simeq\left(\mathcal{O}_{D(J)} \otimes \mathcal{V}\right)^{|J|}$. Because $\nabla$ preserves the filtration $W(J)$ on $\mathcal{O}_{D(J)} \otimes \mathcal{V}$ by the local description in [D1], [Kt] and because $N_{i, D(J)}\left(W(J)_{k}\right) \subset$ $W(J)_{k-1}$ for every $k$ by Lemma $5.12(1)$, we obtain a morphism

$$
\mathrm{Gr}_{k}^{W(J)}\left(\mathcal{O}_{D(J)} \otimes \mathcal{V}\right) \longrightarrow \Omega_{D(J)}^{1}\left(\log D(J) \cap D_{I \backslash J}\right) \otimes \operatorname{Gr}_{k}^{W(J)}\left(\mathcal{O}_{D(J)} \otimes \mathcal{V}\right)
$$

for every $k$. It is denoted by $\nabla_{k}(J)$, or simply $\nabla(J)$. It is easy to see that $\nabla(J)$ is an integrable log connection on $\operatorname{Gr}_{k}^{W(J)}\left(\mathcal{O}_{D(J)} \otimes \mathcal{V}\right)$ satisfying $\nabla(J)\left(F^{p}\right) \subset F^{p-1}$ for every $p$ for the induced filtration $F$ on $\operatorname{Gr}_{k}^{W(J)}\left(\mathcal{O}_{D(J)} \otimes \mathcal{V}\right)$. We can easily see that the residue of $\nabla(J)$ along $D(J) \cap D_{i}$ coincide with $N_{i, D(J \cup\{i\})}$ for $i \in I \backslash J$. Thus $\nabla(J)$ satisfies the condition in 5.3.

5.15. Let $(\mathcal{V}, F, \nabla)$ be as in 5.3 and 5.11 . Then $\left.(\mathcal{V}, F, \nabla)\right|_{X^{*}}$ is a polarizable variation of $\mathbb{R}$-Hodge structure of weight $m$. An integrable logarithmic connection on $\mathcal{V} \otimes \mathcal{V}$ is defined by $\nabla \otimes \mathrm{id}+\mathrm{id} \otimes \nabla$ as usual. Assume that we are given a morphism

$$
S: \mathcal{V} \otimes \mathcal{V} \longrightarrow \mathcal{O}_{X}
$$

satisfying the following:

- $S$ is $(-1)^{m}$-symmetric.

- $S$ is compatible with the connections, where $\mathcal{O}_{X}$ is equipped with the trivial connection $d$. 
- $S\left(F^{p} \mathcal{V} \otimes F^{q} \mathcal{V}\right)=0$ if $p+q>m$.

- $\left.S\right|_{X^{*}}$ underlies a polarization of the variation of $\mathbb{R}$-Hodge structure $\left.(\mathcal{V}, F, \nabla)\right|_{X^{*}}$.

Now we fix a subset $J$ of $I$. Then $S$ induces a morphism

$$
\mathcal{O}_{D(J)} \otimes \mathcal{V} \otimes \mathcal{V} \simeq\left(\mathcal{O}_{D(J)} \otimes \mathcal{V}\right) \otimes\left(\mathcal{O}_{D(J)} \otimes \mathcal{V}\right) \longrightarrow \mathcal{O}_{D(J)}
$$

which is denoted by $S_{J}$.

Lemma 5.16. In the situation above, we have

$$
S_{J}\left(W(K)_{a} \otimes W(K)_{b}\right)=0
$$

for every $K \subset J$ and for every $a, b$ with $a+b<0$.

Proof. We fix a subset $K$ of $J$. It is sufficient to prove that

$$
S_{J}\left(W(K)_{a} \otimes W(K)_{-a-1}\right)=0
$$

for every non-negative integer $a$.

Since $S$ is compatible with the connections, we have

$$
S_{J} \cdot\left(N_{i} \otimes \mathrm{id}+\mathrm{id} \otimes N_{i}\right)=0
$$

for every $i \in J$, from which the equality

$$
S_{J} \cdot\left(N_{K} \otimes \mathrm{id}+\mathrm{id} \otimes N_{K}\right)=0
$$

is obtained. Then we have

$$
\begin{aligned}
S_{J}\left(W(K)_{a}\right. & \left.\otimes W(K)_{-a-1}\right) \\
& =\left(S_{J} \cdot \mathrm{id} \otimes N_{K}^{a+1}\right)\left(W(K)_{a} \otimes W(K)_{a+1}\right) \\
& =(-1)^{a+1}\left(S_{J} \cdot N_{K}^{a+1} \otimes \mathrm{id}\right)\left(W(K)_{a} \otimes W(K)_{a+1}\right) \\
& =(-1)^{a+1} S_{J}\left(W(K)_{-a-2} \otimes W(K)_{a+1}\right) \\
& =(-1)^{a+1+m} S_{J}\left(W(K)_{a+1} \otimes W(K)_{-a-2}\right)
\end{aligned}
$$

by using the equality $W(K)_{-k}=N^{k}\left(W(K)_{k}\right)$ for $k \geq 0$ (see e.g. [SZ, (2.2) Corollary]). Thus we obtain the conclusion by descending induction on $a$.

Corollary 5.17. In the situation above, $S_{J}$ induces a morphism

$$
\mathrm{Gr}_{k}^{W(J)}\left(\mathcal{O}_{D(J)} \otimes \mathcal{V}\right) \otimes \mathrm{Gr}_{-k}^{W(J)}\left(\mathcal{O}_{D(J)} \otimes \mathcal{V}\right) \longrightarrow \mathcal{O}_{D(J)}
$$

for a non-negative integer $k$. 
5.18. In the situation above, we define a morphism

$$
\bar{S}_{k}(J): \mathcal{P}_{k}(J) \otimes \mathcal{P}_{k}(J) \longrightarrow \mathcal{O}_{D(J)}
$$

by $\bar{S}_{k}(J)=S_{J} \cdot\left(\operatorname{id} \otimes N_{J}^{k}\right)$ for every subset $J \subset I$ and for every non-negative integer $k$.

By using the direct sum decomposition

$$
\operatorname{Gr}_{k}^{W(J)}\left(\mathcal{O}_{D(J)} \otimes \mathcal{V}\right)=\bigoplus_{l \geq 0} N^{l}\left(\mathcal{P}_{k+2 l}(J)\right)
$$

for a non-negative integer $k$, we obtain a morphism

$$
S_{k}(J): \operatorname{Gr}_{k}^{W(J)}\left(\mathcal{O}_{D(J)} \otimes \mathcal{V}\right) \otimes \operatorname{Gr}_{k}^{W(J)}\left(\mathcal{O}_{D(J)} \otimes \mathcal{V}\right) \longrightarrow \mathcal{O}_{D(J)}
$$

which is characterized by the following properties:

- For non-negative integers $a, b$ we have

$$
S_{k}(J)\left(N^{a}\left(\mathcal{P}_{k+2 a}(J)\right) \otimes N^{b}\left(\mathcal{P}_{k+2 b}(J)\right)\right)=0
$$

if $a \neq b$.

- The diagram

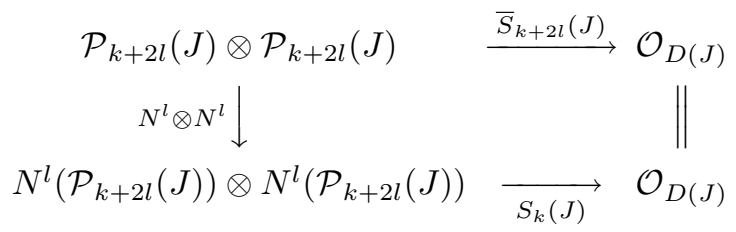

is commutative for every non-negative integer $l$.

For a positive integer $k$, the morphism

$$
S_{-k}(J): \mathrm{Gr}_{-k}^{W(J)}\left(\mathcal{O}_{D(J)} \otimes \mathcal{V}\right) \otimes \mathrm{Gr}_{-k}^{W(J)}\left(\mathcal{O}_{D(J)} \otimes \mathcal{V}\right) \longrightarrow \mathcal{O}_{D(J)}
$$

is defined by identifying $\operatorname{Gr}_{-k}^{W(J)}\left(\mathcal{O}_{D(J)} \otimes \mathcal{V}\right)$ with $\operatorname{Gr}_{k}^{W(J)}\left(\mathcal{O}_{D(J)} \otimes \mathcal{V}\right)$ via the morphism $N(J)^{k}$. More precisely, $S_{-k}(J)$ is the unique morphism such that the diagram

$$
\begin{aligned}
\operatorname{Gr}_{k}^{W(J)}\left(\mathcal{O}_{D(J)} \otimes \mathcal{V}\right) \otimes \operatorname{Gr}_{k}^{W(J)}\left(\mathcal{O}_{D(J)} \otimes \mathcal{V}\right) & \stackrel{S_{k}(J)}{\longrightarrow} \mathcal{O}_{D(J)} \\
N(J)^{k} \otimes N(J)^{k} & \downarrow \\
\operatorname{Gr}_{-k}^{W(J)}\left(\mathcal{O}_{D(J)} \otimes \mathcal{V}\right) \otimes \mathrm{Gr}_{-k}^{W(J)}\left(\mathcal{O}_{D(J)} \otimes \mathcal{V}\right) & \underset{S_{-k}(J)}{\longrightarrow} \mathcal{O}_{D(J)}
\end{aligned}
$$

is commutative. 
The following proposition plays an essential role for the inductive argument in the proof of semipositivity theorem.

Proposition 5.19. In the situation 5.3, 5.11 and 5.15, the data

$$
\left(\mathrm{Gr}_{k}^{W(J)}\left(\mathcal{O}_{D(J)} \otimes \mathcal{V}\right), F, \nabla(J), S_{k}(J)\right)
$$

satisfies the conditions in 5.3, 5.11 and 5.15 again.

Proof. By Lemma 5.5 and 5.14, the pair $\left(\operatorname{Gr}_{k}^{W(J)}\left(\mathcal{O}_{D(J)} \otimes \mathcal{V}\right), \nabla(J)\right)$ satisfies the condition 5.3. As remarked in 5.14, we have $\nabla(J)\left(F^{p}\right) \subset F^{p-1}$ for every $p$. Moreover, the filtration $F$ on $\operatorname{Gr}_{k}^{W(J)}\left(\mathcal{O}_{D(J)} \otimes \mathcal{V}\right)$ satisfies the condition $((m+k) \mathrm{MH})$ by Corollary 5.13 .

By definition, the morphism

$$
S_{J}: \operatorname{Gr}_{k}^{W(J)}\left(\mathcal{O}_{D(J)} \otimes \mathcal{V}\right) \otimes \operatorname{Gr}_{-k}^{W(J)}\left(\mathcal{O}_{D(J)} \otimes \mathcal{V}\right)
$$

is compatible with the connections on both sides. Therefore $\bar{S}_{k}(J)$ is compatible with the connections because

$$
N_{J}: \operatorname{Gr}_{k}^{W(J)}\left(\mathcal{O}_{D(J)} \otimes \mathcal{V}\right) \longrightarrow \operatorname{Gr}_{k-2}^{W(J)}\left(\mathcal{O}_{D(J)} \otimes \mathcal{V}\right)
$$

is compatible with the connection $\nabla(J)$ on the both sides. Thus $S_{k}(J)$ is compatible with the connection. Moreover we can check the equality

$$
S_{k}(J)\left(F^{p} \otimes F^{q}\right)=0
$$

for $p+q>m+k$ by using $N_{J}^{k}\left(F^{q}\right) \subset F^{q-k}$.

There exists an open subset $U$ of $D(J)^{*}$ such that $\operatorname{Gr}_{F}^{p} \operatorname{Gr}_{k}^{W(J)}\left(\mathcal{O}_{D(J)} \otimes \mathcal{V}\right)$ is a locally free $\mathcal{O}_{D(J)}$-module of finite rank for every $p$ and that $D(J) \backslash U$ is a nowhere dense closed analytic subspace of $D(J)$.

By the local description as in Deligne [D1], Katz [Kt] and by the property (1) in Lemma 5.12 , we can easily check that $\left.\operatorname{Ker}\left(\nabla_{k}(J)\right)\right|_{D(J)^{*}}$ admits an $\mathbb{R}$-structure, that is, there exists a local system $\mathbb{V}_{k}(J)$ of finite dimensional $\mathbb{R}$-vector spaces with the property $\left.\mathbb{C} \otimes \mathbb{V}_{k}(J) \simeq \operatorname{Ker}\left(\nabla_{k}(J)\right)\right|_{D(J)^{*}}$. Then the data

$$
\left.\left(\mathbb{V}_{k}(J),\left(\operatorname{Gr}_{k}^{W(J)}\left(\mathcal{O}_{D(J)} \otimes \mathcal{V}\right), F\right), \nabla(J), S_{k}(J)\right)\right|_{U}
$$

is a polarized variation of $\mathbb{R}$-Hodge structure of weight $m+k$, by Schmid [Sc]. By Lemma $5.10, \operatorname{Gr}_{F}^{p} \operatorname{Gr}_{k}^{W(J)}\left(\mathcal{O}_{D(J)} \otimes \mathcal{V}\right)$ turns out to be locally free for every $k, p$ and then

$$
\left.\left(\mathrm{Gr}_{k}^{W(J)}\left(\mathcal{O}_{D(J)} \otimes \mathcal{V}\right), F, \nabla(J), S_{k}(J)\right)\right|_{D(J)^{*}}
$$

underlies a polarized variation of $\mathbb{R}$-Hodge structure of weight $m+k$ as desired. By the continuity, $S_{k}(J)$ is $(-1)^{m+k}$-symmetric. 
Let us recall the definition of semipositive vector bundles in the sense of Fujita-Kawamata. Example 8.2 below helps us understand the Fujita-Kawamata semipositivity.

Definition 5.20 (Semipositivity). A locally free sheaf (or a vector bundle) $\mathcal{E}$ of finite rank on a complete algebraic variety $X$ is said to be semipositive if for every smooth curve $C$, for every morphism $\varphi: C \longrightarrow X$, and for every quotient invertible sheaf (or line bundle) $\mathcal{Q}$ of $\varphi^{*} \mathcal{E}$, we have $\operatorname{deg}_{C} \mathcal{Q} \geq 0$.

It is easy to see that $\mathcal{E}$ is semipositive if and only if $\mathcal{O}_{\mathbb{P}_{X}(\mathcal{E})}(1)$ is nef where $\mathcal{O}_{\mathbb{P}_{X}(\mathcal{E})}(1)$ is the tautological line bundle on $\mathbb{P}_{X}(\mathcal{E})$.

The following theorem is the main result of this section (cf. [Kw1, Theorem 5]). It is a completely Hodge theoretic result.

Theorem 5.21 (Semipositivity theorem). Let $X$ be a smooth complete complex variety, $D$ a simple normal crossing divisor on $X, \mathcal{V}$ a locally free $\mathcal{O}_{X}$-module of finite rank equipped with a finite increasing filtration $W$ and a finite decreasing filtration $F$. We assume the following:

(1) $F^{a} \mathcal{V}=\mathcal{V}$ and $F^{b+1} \mathcal{V}=0$ for some $a<b$

(2) $\operatorname{Gr}_{F}^{p} \operatorname{Gr}_{m}^{W} \mathcal{V}$ is a locally free $\mathcal{O}_{X}$-module of finite rank for all $m, p$.

(3) For all $m, \mathrm{Gr}_{m}^{W} \mathcal{V}$ admits an integrable logarithmic connection $\nabla_{m}$ with the nilpotent residue morphisms which satisfies the conditions

$$
\nabla_{m}\left(F^{p} \mathrm{Gr}_{m}^{W} \mathcal{V}\right) \subset \Omega_{X}^{1}(\log D) \otimes F^{p-1} \mathrm{Gr}_{m}^{W} \mathcal{V}
$$

for all $p$.

(4) The triple $\left.\left(\operatorname{Gr}_{m}^{W} \mathcal{V}, F \mathrm{Gr}_{m}^{W} \mathcal{V}, \nabla_{m}\right)\right|_{X \backslash D}$ underlies a polarizable variation of $\mathbb{R}$ Hodge structure of weight $m$ for every integer $m$.

Then $\left(\operatorname{Gr}_{F}^{a} \mathcal{V}\right)^{*}$ and $F^{b} \mathcal{V}$ are semipositive.

Proof. Since a vector bundle which is an extension of two semipositive vector bundles is also semipositive, we may assume that the given $\mathcal{V}$ is pure of weight $m$, that is, $W_{m} \mathcal{V}=\mathcal{V}, W_{m-1} \mathcal{V}=0$ for an integer $m$, without loss of generality. Then $\mathcal{V}$ carries an integrable logarithmic connection $\nabla$ whose residue morphisms are nilpotent. Thus the data $(\mathcal{V}, F, \nabla)$ satisfies the conditions in 5.3 and 5.11 . Note that $\mathcal{V}$ is the canonical extension of $\left.\mathcal{V}\right|_{X \backslash D}$ because the residue morphisms of $\nabla$ are nilpotent.

By the assumption (5.21) above, $\left.\mathcal{V}\right|_{X \backslash D}$ carries a polarization which extends to a morphism

$$
S: \mathcal{V} \otimes \mathcal{V} \longrightarrow \mathcal{O}_{X}
$$


by functoriality of the canonical extensions. We can easily see that the data $(\mathcal{V}, F, \nabla)$ and $S$ satisfies the conditions in 5.3, 5.11 and 5.15.

For the case of $\operatorname{dim} X=1$, we obtain the conclusion by Zucker [Z] (see also Kawamata [Kw1] and the proof of [Ko3, Theorem 5.20]).

Next, we study the case of $\operatorname{dim} X>1$. Let $\varphi: C \longrightarrow X$ be a morphism from a smooth projective curve. The irreducible decomposition of $D$ is denoted by $D=\sum_{i \in I} D_{i}$ as in 5.3. We set $J=\left\{i \in I ; \varphi(C) \subset D_{i}\right\} \subset I$. Then $\varphi(C) \subset D(J)$, $\varphi(C) \cap D(J)^{*} \neq \emptyset$ and $\varphi^{*} D_{I \backslash J}$ is an effective divisor on $C$. By Proposition 5.19, the locally free sheaf $\mathcal{O}_{D(J)} \otimes \mathcal{V}$ with the finite increasing filtration $W(J)$ and the finite decreasing filtration $F$ satisfies the assumptions (1)-(4) for $D(J)$ with the simple normal crossing divisor $D(J) \cap D_{I \backslash J}$. Therefore $\varphi^{*} \mathcal{V}=\varphi^{*}\left(\mathcal{O}_{D(J)} \otimes \mathcal{V}\right)$ with the induced filtrations $W$ and $F$ satisfies the assumptions (1)-(4) for $C$ with the effective divisor $\varphi^{*} D_{I \backslash J}$. Then we conclude the desired semipositivity by the case of $\operatorname{dim} X=1$.

Remark 5.22. In Theorem 5.21, if $X$ is not complete, then we have the following statement. Let $V$ be a complete subvariety of $X$. Then $\left.\left(\operatorname{Gr}_{F}^{a} \mathcal{V}\right)^{*}\right|_{V}$ and $\left.\left(F^{b} \mathcal{V}\right)\right|_{V}$ are semipositive locally free sheaves on $V$. It is obvious by the proof of Theorem 5.21 .

Corollary 5.23. Let $X$ and $D$ be as in Theorem 5.21. Assume that we are given an admissible graded polarizable variation of $\mathbb{R}$-mixed Hodge structure $V=((\mathbb{V}, W), F)$ on $X \backslash D$ of unipotent monodromy. We assume the conditions $F^{a} \mathcal{V}=\mathcal{V}$ and $F^{b+1} \mathcal{V}=0$. The canonical extensions of $\mathcal{V}=\mathcal{O}_{X \backslash D} \otimes \mathbb{V}$ and of $W_{k} \mathcal{V}=\mathcal{O}_{X \backslash D} \otimes W_{k}$ are denoted by $\widetilde{\mathcal{V}}$ and by $W_{k} \widetilde{\mathcal{V}}$ for all $k$. As stated in Proposition 3.12, the Hodge filtration $F$ extends to $\widetilde{\mathcal{V}}$ such that $\operatorname{Gr}_{F}^{p} \operatorname{Gr}_{k}^{W} \widetilde{\mathcal{V}}$ is locally free of finite rank for all $k, p$. Then $\left(\mathrm{Gr}_{F}^{a} \widetilde{\mathcal{V}}\right)^{*}$ and $F^{b} \widetilde{\mathcal{V}}$ are semipositive.

We learned the following remark from Hacon.

Remark 5.24. The proof of the semipositivity theorem in [Ko5, Theorem 8.10.12] contains some ambiguities. In the same notation as in [Ko5, Theorem 8.10.12], if $D$ is a simple normal crossing divisor but is not a smooth divisor, then it is not clear how to express $R^{m} f_{*} \omega_{X / Y}(D)$ as an extension of $R^{m} f_{*} \omega_{D_{J} / Y}$ 's. The case when $D=F$ is a smooth divisor is treated in the proof of [Ko5, Theorem 8.10.12]. The same argument does not seem to be sufficient for the general case.

Fortunately, [F4, Theorem 3.9] is sufficient for all applications in [Ko5] (see also [FG2]). For some related topics, see [FFS]. 


\section{§6. Vanishing and torsion-free theorems}

In this section, we discuss some generalizations of torsion-free and vanishing theorems for quasi-projective simple normal crossing pairs.

First, let us recall the following very useful lemma. For a proof, see, for example, [F14, Lemma 3.3].

Lemma 6.1 (Relative vanishing lemma). Let $f: Y \longrightarrow X$ be a proper morphism from a simple normal crossing pair $(Y, \Delta)$ to an algebraic variety $X$ such that $\Delta$ is a boundary $\mathbb{R}$-divisor on $Y$. We assume that $f$ is an isomorphism at the generic point of any stratum of the pair $(Y, \Delta)$. Let $L$ be a Cartier divisor on $Y$ such that $L \sim_{\mathbb{R}} K_{Y}+\Delta$. Then $R^{q} f_{*} \mathcal{O}_{Y}(L)=0$ for every $q>0$.

As an application of Lemma 6.1, we obtain Lemma 6.2. We will use it several times in Section 7.

Lemma 6.2 (cf. [F13, Lemma 2.7]). Let $\left(V_{1}, D_{1}\right)$ and $\left(V_{2}, D_{2}\right)$ be simple normal crossing pairs such that $D_{1}$ and $D_{2}$ are reduced. Let $f: V_{1} \longrightarrow V_{2}$ be a proper morphism. Assume that there is a Zariski open subset $U_{1}$ (resp. $\left.U_{2}\right)$ of $V_{1}$ (resp. $\left.V_{2}\right)$ such that $U_{1}$ (resp. $\left.U_{2}\right)$ contains the generic point of any stratum of $\left(V_{1}, D_{1}\right)$ (resp. $\left.\left(V_{2}, D_{2}\right)\right)$ and that $f$ induces an isomorphism between $U_{1}$ and $U_{2}$. Then $R^{i} f_{*} \omega_{V_{1}}\left(D_{1}\right)=0$ for every $i>0$ and $f_{*} \omega_{V_{1}}\left(D_{1}\right) \simeq \omega_{V_{2}}\left(D_{2}\right)$. By Grothendieck duality, we obtain that $R^{i} f_{*} \mathcal{O}_{V_{1}}\left(-D_{1}\right)=0$ for every $i>0$ and $f_{*} \mathcal{O}_{V_{1}}\left(-D_{1}\right) \simeq$ $\mathcal{O}_{V_{2}}\left(-D_{2}\right)$.

Proof. We can write

$$
K_{V_{1}}+D_{1}=f^{*}\left(K_{V_{2}}+D_{2}\right)+E
$$

such that $E$ is $f$-exceptional. We consider the following commutative diagram

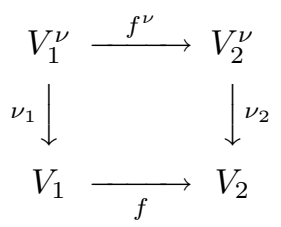

where $\nu_{1}: V_{1}^{\nu} \longrightarrow V_{1}$ and $\nu_{2}: V_{2}^{\nu} \longrightarrow V_{2}$ are the normalizations. We can write $K_{V_{1}^{\nu}}+\Theta_{1}=\nu_{1}^{*}\left(K_{V_{1}}+D_{1}\right)$ and $K_{V_{2}^{\nu}}+\Theta_{2}=\nu_{2}^{*}\left(K_{V_{2}}+D_{2}\right)$. By pulling back $K_{V_{1}}+D_{1}=f^{*}\left(K_{V_{2}}+D_{2}\right)+E$ to $V_{1}^{\nu}$ by $\nu_{1}$, we have

$$
K_{V_{1}^{\nu}}+\Theta_{1}=\left(f^{\nu}\right)^{*}\left(K_{V_{2}^{\nu}}+\Theta_{2}\right)+\nu_{1}^{*} E
$$

Note that $V_{2}^{\nu}$ is smooth and $\Theta_{2}$ is a reduced simple normal crossing divisor on $V_{2}^{\nu}$. By assumption, $f^{\nu}$ is an isomorphism over the generic point of any lc center of 
the pair $\left(V_{2}^{\nu}, \Theta_{2}\right)$ (cf. 1.10). Therefore, $\nu_{1}^{*} E$ is effective since $K_{V_{2}^{\nu}}+\Theta_{2}$ is Cartier. Thus, we obtain that $E$ is effective. Since $V_{2}$ satisfies Serre's $S_{2}$ condition, we can check that $\mathcal{O}_{V_{2}} \simeq f_{*} \mathcal{O}_{V_{1}}$ and $f_{*} \mathcal{O}_{V_{1}}\left(K_{V_{1}}+D_{1}\right) \simeq \mathcal{O}_{V_{2}}\left(K_{V_{2}}+D_{2}\right)$. On the other hand, we obtain $R^{i} f_{*} \mathcal{O}_{V_{1}}\left(K_{V_{1}}+D_{1}\right)=0$ for every $i>0$ by Lemma 6.1 . Therefore, $R f_{*} \mathcal{O}_{V_{1}}\left(K_{V_{1}}+D_{1}\right) \simeq \mathcal{O}_{V_{2}}\left(K_{V_{2}}+D_{2}\right)$ in the derived category of coherent sheaves on $V_{2}$. Since $V_{1}$ and $V_{2}$ are Gorenstein, we have

$$
\begin{aligned}
R f_{*} \mathcal{O}_{V_{1}}\left(-D_{1}\right) & \simeq R \mathcal{H o m}\left(R f_{*} \omega_{V_{1}}^{\bullet}\left(D_{1}\right), \omega_{V_{2}}^{\bullet}\right) \\
& \simeq R \mathcal{H o m}\left(R f_{*} \omega_{V_{1}}\left(D_{1}\right), \omega_{V_{2}}\right) \\
& \simeq R \mathcal{H} \operatorname{Hom}\left(\omega_{V_{2}}\left(D_{2}\right), \omega_{V_{2}}\right) \simeq \mathcal{O}_{V_{2}}\left(-D_{2}\right)
\end{aligned}
$$

in the derived category of coherent sheaves on $V_{2}$ by Grothendieck duality. Therefore, we have $R^{i} f_{*} \mathcal{O}_{V_{1}}\left(-D_{1}\right)=0$ for every $i>0$ and $f_{*} \mathcal{O}_{V_{1}}\left(-D_{1}\right) \simeq \mathcal{O}_{V_{2}}\left(-D_{2}\right)$.

Next, we prove the following theorem, which was proved for embedded simple normal crossing pairs in [F7, Theorem 2.39] and [F7, Theorem 2.47]. We note that we do not assume the existence of ambient spaces in Theorem 6.3. However, we need the assumption that $X$ is quasi-projective.

Theorem 6.3 (cf. [F7, Theorem 2.39 and Theorem 2.47]). Let $(X, B)$ be a quasiprojective simple normal crossing pair such that $B$ is a boundary $\mathbb{R}$-divisor on $X$. Let $f: X \longrightarrow Y$ be a proper morphism between algebraic varieties and let $L$ be a Cartier divisor on $X$. Let $q$ be an arbitrary integer. Then we have the following properties.

(i) Assume that $L-\left(K_{X}+B\right)$ is f-semi-ample, that is, $L-\left(K_{X}+B\right)=\sum_{i} a_{i} D_{i}$ where $D_{i}$ is an $f$-semi-ample Cartier divisor on $X$ and $a_{i}$ is a positive real number for every $i$. Then every associated prime of $R^{q} f_{*} \mathcal{O}_{X}(L)$ is the generic point of the $f$-image of some stratum of $(X, B)$.

(ii) Let $\pi: Y \longrightarrow Z$ be a proper morphism. We assume that $L-\left(K_{X}+B\right) \sim_{\mathbb{R}} f^{*} A$ for some $\mathbb{R}$-Cartier $\mathbb{R}$-divisor $A$ on $Y$ such that $A$ is nef and log big over $Z$ with respect to $f:(X, B) \longrightarrow Y$ (see $\left[F\right.$, Definition 2.46]). Then $R^{q} f_{*} \mathcal{O}_{X}(L)$ is $\pi_{*}$-acyclic, that is, $R^{p} \pi_{*} R^{q} f_{*} \mathcal{O}_{X}(L)=0$ for every $p>0$.

Proof. Since $X$ is quasi-projective, we can embed $X$ into a smooth projective variety $V$. By Lemma 6.5 below, we can replace $(X, B)$ and $L$ with $\left(X_{k}, B_{k}\right)$ and $\sigma^{*} L$ and assume that there exists an $\mathbb{R}$-divisor $D$ on $V$ such that $B=\left.D\right|_{X}$. Then, by using Bertini's theorem, we can take a general complete intersection $W \subset V$ such that $\operatorname{dim} W=\operatorname{dim} X+1, X \subset W$, and $W$ is smooth at the generic point 
of every stratum of $(X, B)$ (cf. the proof of [Ko6, Proposition 10.59]). We take a suitable resolution $\psi: M \longrightarrow W$ with the following properties.

(A) The strict transform $X^{\prime}$ of $X$ is a simple normal crossing divisor on $M$.

(B) We can write

$$
K_{X^{\prime}}+B^{\prime}=\varphi^{*}\left(K_{X}+B\right)+E
$$

such that $\varphi=\left.\psi\right|_{X^{\prime}},\left(X^{\prime}, B^{\prime}-E\right)$ is a globally embedded simple normal crossing pair (cf. Definition 2.3), $B^{\prime}$ is a boundary $\mathbb{R}$-divisor on $X^{\prime},\lceil E\rceil$ is effective and $\varphi$-exceptional, and the $\varphi$-image of every stratum of $\left(X^{\prime}, B^{\prime}\right)$ is a stratum of $(X, B)$.

(C) $\varphi$ is an isomorphism over the generic point of every stratum of $(X, B)$.

(D) $\varphi$ is an isomorphism at the generic point of every stratum of $\left(X^{\prime}, B^{\prime}\right)$.

Then

$$
\begin{gathered}
K_{X^{\prime}}+B^{\prime}+\{-E\}=\varphi^{*}\left(K_{X}+B\right)+\lceil E\rceil, \\
\varphi_{*} \mathcal{O}_{X^{\prime}}\left(\varphi^{*} L+\lceil E\rceil\right) \simeq \mathcal{O}_{X}(L),
\end{gathered}
$$

and

$$
R^{q} \varphi_{*} \mathcal{O}_{X^{\prime}}\left(\varphi^{*} L+\lceil E\rceil\right)=0
$$

for every $q>0$ by Lemma 6.1 . We note that

$$
\varphi^{*} L+\lceil E\rceil-\left(K_{X^{\prime}}+B^{\prime}+\{-E\}\right)=\varphi^{*}\left(L-\left(K_{X}+B\right)\right)
$$

and that $\varphi$ is an isomorphism at the generic point of every stratum of $\left(X^{\prime}, B^{\prime}+\right.$ $\{-E\})$.

Therefore, by replacing $(X, B)$ and $L$ with $\left(X^{\prime}, B^{\prime}+\{-E\}\right)$ and $\varphi^{*} L+\lceil E\rceil$, we may assume that $(X, B)$ is a quasi-projective globally embedded simple normal crossing pair (cf. Definition 2.3). In this case, the claims have already been established by [F7, Theorem 2.39] and [F7, Theorem 2.47].

For some generalizations of Theorem 6.3 for semi log canonical pairs, see [F15].

Remark 6.4. Theorem 6.3 (i) is contained in [F14, Theorem 1.1 (i)]. In [F14, Theorem 1.1], $X$ is not assumed to be quasi-projective. On the other hand, we do not know how to remove the quasi-projectivity of $X$ from Theorem 6.3 (ii).

By direct calculations, we can obtain the following elementary lemma. It was used in the proof of Theorem 6.3.

Lemma 6.5 (cf. [F7, Lemma 3.60]). Let $(X, B)$ be a simple normal crossing pair such that $B$ is a boundary $\mathbb{R}$-divisor. Let $V$ be a smooth variety such that $X \subset V$. 
Then we can construct a sequence of blow-ups

$$
V_{k} \longrightarrow V_{k-1} \longrightarrow \cdots \longrightarrow V_{0}=V
$$

with the following properties.

(1) $\sigma_{i+1}: V_{i+1} \longrightarrow V_{i}$ is the blow-up along a smooth irreducible component of $\operatorname{Supp} B_{i}$ for every $i \geq 0$.

(2) We put $X_{0}=X, B_{0}=B$, and $X_{i+1}$ is the strict transform of $X_{i}$ for every $i \geq 0$.

(3) We put $K_{X_{i+1}}+B_{i+1}=\sigma_{i+1}^{*}\left(K_{X_{i}}+B_{i}\right)$ for every $i \geq 0$.

(4) There exists an $\mathbb{R}$-divisor $D$ on $V_{k}$ such that $\left.D\right|_{X_{k}}=B_{k}$ and $B_{k}$ is a boundary $\mathbb{R}$-divisor on $X_{k}$.

(5) $\sigma_{*} \mathcal{O}_{X_{k}} \simeq \mathcal{O}_{X}$ and $R^{q} \sigma_{*} \mathcal{O}_{X_{k}}=0$ for every $q>0$, where $\sigma: V_{k} \longrightarrow V_{k-1} \longrightarrow$ $\cdots \longrightarrow V_{0}=V$.

Proof. All we have to do is to check the property (5). We note that

$$
\sigma_{i+1 *} \mathcal{O}_{V_{i+1}}\left(K_{V_{i+1}}\right) \simeq \mathcal{O}_{V_{i+1}}\left(K_{V_{i+1}}\right)
$$

and $R^{q} \sigma_{i+1 *} \mathcal{O}_{V_{i+1}}\left(K_{V_{i+1}}\right)=0$ for every $q$ and for each step $\sigma_{i+1}: V_{i+1} \longrightarrow V_{i}$ by Lemma 6.2. Therefore we obtain $R^{q} \sigma_{*} \mathcal{O}_{X_{k}}\left(K_{X_{k}}\right)=0$ for every $q>0$ and $\sigma_{*} \mathcal{O}_{X_{k}}\left(K_{X_{k}}\right) \simeq \mathcal{O}_{X}\left(K_{X}\right)$. Thus by Grothendieck duality we obtain $R^{q} \sigma_{*} \mathcal{O}_{X_{k}}=0$ for every $q>0$ and $\sigma_{*} \mathcal{O}_{X_{k}} \simeq \mathcal{O}_{X}$ as in the proof of Lemma 6.2.

As a special case of Theorem 6.3 (i), we have:

Corollary 6.6 (Torsion-freeness). Let $(X, D)$ be a quasi-projective simple normal crossing pair such that $D$ is reduced and let $f: X \rightarrow Y$ be a projective surjective morphism onto a smooth algebraic variety $Y$. Assume that every stratum of $(X, D)$ is dominant onto $Y$. Then $R^{i} f_{*} \omega_{X / Y}(D)$ is torsion-free for every $i$.

Proof. It is sufficient to prove that $R^{i} f_{*} \mathcal{O}_{X}\left(K_{X}+D\right)$ is torsion-free for every $i$ since $\mathcal{O}_{Y}\left(K_{Y}\right)$ is locally free. By Theorem 6.3 (i), every associated prime of $R^{i} f_{*} \mathcal{O}_{X}\left(K_{X}+D\right)$ is the generic point of $Y$ for every $i$. This means that $R^{i} f_{*} \mathcal{O}_{X}\left(K_{X}+\right.$ $D)$ is torsion-free for every $i$.

We will use it in Section 7.

\section{$\S 7$. Higher direct images of log canonical divisors}

This section is the main part of this paper. The following theorem is our main theorem (cf. [Kw1, Theorem 5], [Ko2, Theorem 2.6], [N1, Theorem 1], [F4, Theo- 
rems 3.4 and 3.9], and [Kw3, Theorem 1.1]), which is a natural generalization of the Fujita-Kawamata semipositivity theorem for simple normal crossing pairs.

Theorem 7.1. Let $(X, D)$ be a simple normal crossing pair such that $D$ is reduced and let $f: X \longrightarrow Y$ be a projective surjective morphism onto a smooth algebraic variety $Y$. Assume that every stratum of $(X, D)$ is dominant onto $Y$. Let $\Sigma$ be a simple normal crossing divisor on $Y$ such that every stratum of $(X, D)$ is smooth over $Y^{*}=Y \backslash \Sigma$. We put $X^{*}=f^{-1}\left(Y^{*}\right), D^{*}=\left.D\right|_{X^{*}}$, and $d=\operatorname{dim} X-\operatorname{dim} Y$. Let $\iota: X^{*} \backslash D^{*} \longrightarrow X^{*}$ be the natural open immersion. Then we obtain

(1) $R^{k}\left(\left.f\right|_{X^{*}}\right)_{*} \iota_{!} \mathbb{Q}_{X^{*} \backslash D^{*}} \simeq R^{k}\left(\left.f\right|_{\left.X^{*} \backslash D^{*}\right) !} \mathbb{Q}_{X^{*} \backslash D^{*}}\right.$ underlies a graded polarizable variation of $\mathbb{Q}$-mixed Hodge structure on $Y^{*}$ for every $k$. Moreover, it is admissible.

We put $\mathcal{V}_{Y^{*}}^{k}=R^{k}\left(\left.f\right|_{X^{*}}\right)_{*} \iota_{!} \mathbb{Q}_{X^{*} \backslash D^{*}} \otimes \mathcal{O}_{Y^{*}}$ for every $k$. Let

$$
\cdots \subset F^{p+1}\left(\mathcal{V}_{Y^{*}}^{k}\right) \subset F^{p}\left(\mathcal{V}_{Y^{*}}^{k}\right) \subset F^{p-1}\left(\mathcal{V}_{Y^{*}}^{k}\right) \subset \cdots
$$

be the Hodge filtration. We assume that all the local monodromies on the local system $R^{d-i}\left(\left.f\right|_{X^{*}}\right)_{*} \iota \mathbb{Q}_{X^{*} \backslash D^{*}}$ around $\Sigma$ are unipotent. Then we obtain

(2) $R^{d-i} f_{*} \mathcal{O}_{X}(-D)$ is isomorphic to the canonical extension of

$$
\operatorname{Gr}_{F}^{0}\left(\mathcal{V}_{Y^{*}}^{d-i}\right)=F^{0}\left(\mathcal{V}_{Y^{*}}^{d-i}\right) / F^{1}\left(\mathcal{V}_{Y^{*}}^{d-i}\right) .
$$

It is denoted by $\operatorname{Gr}_{F}^{0}\left(\mathcal{V}_{Y}^{d-i}\right)$. In particular, $R^{d-i} f_{*} \mathcal{O}_{X}(-D)$ is locally free.

By Grothendieck duality, we obtain

(3) $R^{i} f_{*} \omega_{X / Y}(D)$ is isomorphic to the canonical extension of

$$
\left(\operatorname{Gr}_{F}^{0}\left(\mathcal{V}_{Y^{*}}^{d-i}\right)\right)^{*}=\mathcal{H}_{o m} \text { O }_{Y^{*}}\left(\operatorname{Gr}_{F}^{0}\left(\mathcal{V}_{Y^{*}}^{d-i}\right), \mathcal{O}_{Y^{*}}\right) .
$$

Thus, we have $R^{i} f_{*} \omega_{X / Y}(D) \simeq\left(\operatorname{Gr}_{F}^{0}\left(\mathcal{V}_{Y}^{d-i}\right)\right)^{*}$. In particular, $R^{i} f_{*} \omega_{X / Y}(D)$ is locally free.

(4) We further assume that $Y$ is complete. Then $R^{i} f_{*} \omega_{X / Y}(D)$ is semipositive.

Even the following very special case of Theorem 7.1 has never been checked before. It does not follow from [Kw3, Theorem 1.1].

Corollary 7.2. Let $f: X \longrightarrow Y$ be a projective morphism from a simple normal crossing variety $X$ to a smooth complete algebraic variety $Y$. Assume that every stratum of $X$ is smooth over $Y$. Then $R^{i} f_{*} \omega_{X / Y}$ is a semipositive locally free sheaf for every $i$. 
It is natural to prove Theorem 7.3, which is a slight generalization of (2) and (3) in Theorem 7.1, simultaneously with Theorem 7.1.

Theorem 7.3 (cf. [Ko2, Theorem 2.6]). We use the same notation and assumptions as in Theorem 7.1. We do not assume that the local monodromies on the local system $R^{d-i}\left(\left.f\right|_{X^{*}}\right)_{*} \iota ! \mathbb{Q}_{X^{*} \backslash D^{*}}$ around $\Sigma$ are unipotent. Then we obtain the following properties.

(a) $R^{d-i} f_{*} \mathcal{O}_{X}(-D)$ is isomorphic to the lower canonical extension of

$$
\operatorname{Gr}_{F}^{0}\left(\mathcal{V}_{Y^{*}}^{d-i}\right)=F^{0}\left(\mathcal{V}_{Y^{*}}^{d-i}\right) / F^{1}\left(\mathcal{V}_{Y^{*}}^{d-i}\right)
$$

In particular, $R^{d-i} f_{*} \mathcal{O}_{X}(-D)$ is locally free.

By Grothendieck duality, we obtain

(b) $R^{i} f_{*} \omega_{X / Y}(D)$ is isomorphic to the upper canonical extension of

$$
\left(\operatorname{Gr}_{F}^{0}\left(\mathcal{V}_{Y^{*}}^{d-i}\right)\right)^{*}=\mathcal{H o m}_{\mathcal{O}_{Y^{*}}}\left(\operatorname{Gr}_{F}^{0}\left(\mathcal{V}_{Y^{*}}^{d-i}\right), \mathcal{O}_{Y^{*}}\right) .
$$

In particular, $R^{i} f_{*} \omega_{X / Y}(D)$ is locally free.

Before we start the proof of Theorem 7.1 and Theorem 7.3, we give a remark on the canonical extensions.

Remark 7.4 (Upper and lower canonical extensions of Hodge bundles). Let ${ }^{l} \mathcal{V}_{Y^{*}}^{k}$ (resp. ${ }^{u} \mathcal{V}_{Y^{*}}^{k}$ ) be the Deligne extension of $\mathcal{V}_{Y^{*}}^{k}$ on $Y$ such that the eigen values of the residue of the connection are contained in $[0,1)$ (resp. $(-1,0])$. We call it the lower canonical extension (resp. upper canonical extension) of $\mathcal{V}_{Y^{*}}^{k}$ following $[\mathrm{Ko} 2$, Definition 2.3]. If the local monodromies on $R^{k}\left(\left.f\right|_{X^{*}}\right)_{*} \iota ! \mathbb{Q}_{X^{*} \backslash D^{*}} \simeq R^{k}\left(\left.f\right|_{X^{*} \backslash D^{*}}\right) ! \mathbb{Q}_{X^{*} \backslash D^{*}}$ around $\Sigma$ are unipotent, then

$$
{ }^{l} \mathcal{V}_{Y^{*}}^{k}={ }^{u} \mathcal{V}_{Y^{*}}^{k}
$$

holds. In this case, we set

$$
\mathcal{V}_{Y}^{k}={ }^{l} \mathcal{V}_{Y^{*}}^{k}={ }^{u} \mathcal{V}_{Y^{*}}^{k}
$$

and call it the canonical extension of $\mathcal{V}_{Y^{*}}^{k}$. Let $j: Y^{*} \longrightarrow Y$ be the natural open immersion. We set

$$
{ }^{l} F^{p}\left(\mathcal{V}_{Y^{*}}^{k}\right)=j_{*} F^{p}\left(\mathcal{V}_{Y^{*}}^{k}\right) \cap{ }^{l} \mathcal{V}_{Y^{*}}^{k}
$$

and call it the lower canonical extension of $F^{p}\left(\mathcal{V}_{Y^{*}}^{k}\right)$ on $Y$. We can define the upper canonical extension ${ }^{u} F^{p}\left(\mathcal{V}_{Y^{*}}^{k}\right)$ of $F^{p}\left(\mathcal{V}_{Y^{*}}^{k}\right)$ on $Y$ similarly. As above, when the local monodromies on $R^{k}\left(\left.f\right|_{X^{*}}\right)_{*} \iota ! \mathbb{Q}_{X^{*} \backslash D^{*}}$ around $\Sigma$ are unipotent, we write $F^{p}\left(\mathcal{V}_{Y}^{k}\right)$ to denote ${ }^{l} F^{p}\left(\mathcal{V}_{Y^{*}}^{k}\right)={ }^{u} F^{p}\left(\mathcal{V}_{Y^{*}}^{k}\right)$ and call it the canonical extension of $F^{p}\left(\mathcal{V}_{Y^{*}}^{k}\right)$. Theorem 7.3 (a) means that the following short exact sequence

$$
0 \longrightarrow F^{1}\left(\mathcal{V}_{Y^{*}}^{d-i}\right) \longrightarrow F^{0}\left(\mathcal{V}_{Y^{*}}^{d-i}\right) \longrightarrow \operatorname{Gr}_{F}^{0}\left(\mathcal{V}_{Y^{*}}^{d-i}\right) \longrightarrow 0
$$


is extended to the short exact sequence

$$
0 \longrightarrow{ }^{l} F^{1}\left(\mathcal{V}_{Y^{*}}^{d-i}\right) \longrightarrow{ }^{l} F^{0}\left(\mathcal{V}_{Y^{*}}^{d-i}\right) \longrightarrow R^{d-i} f_{*} \mathcal{O}_{X}(-D) \longrightarrow 0
$$

Let us consider the dual variation of mixed Hodge structure (cf. Remark 3.15). The dual local system of $R^{k}\left(\left.f\right|_{X^{*}}\right)_{*} \iota_{!} \mathbb{Q}_{X^{*} \backslash D^{*}}$ underlies $\left(\mathcal{V}_{Y^{*}}^{k}\right)^{*}$. The locally free sheaf $\left(\mathcal{V}_{Y^{*}}^{k}\right)^{*}$ carries the Hodge filtration $F$ defined in Remark 3.15. Theorem 7.3 (b) means that the short exact sequence

$$
0 \longrightarrow F^{1}\left(\left(\mathcal{V}_{Y^{*}}^{d-i}\right)^{*}\right) \longrightarrow F^{0}\left(\left(\mathcal{V}_{Y^{*}}^{d-i}\right)^{*}\right) \longrightarrow \operatorname{Gr}_{F}^{0}\left(\left(\mathcal{V}_{Y^{*}}^{d-i}\right)^{*}\right) \longrightarrow 0
$$

is extended to the short exact sequence

$$
0 \rightarrow{ }^{u} F^{1}\left(\left(\mathcal{V}_{Y^{*}}^{d-i}\right)^{*}\right) \rightarrow{ }^{u} F^{0}\left(\left(\mathcal{V}_{Y^{*}}^{d-i}\right)^{*}\right) \rightarrow R^{i} f_{*} \omega_{X / Y}(D) \rightarrow 0
$$

We note that

$$
\operatorname{Gr}_{F}^{-p}\left(\left(\mathcal{V}_{Y^{*}}^{d-i}\right)^{*}\right) \simeq\left(\operatorname{Gr}_{F}^{p}\left(\mathcal{V}_{Y^{*}}^{d-i}\right)\right)^{*}
$$

for every $p$ as in Remark 3.15. We also note that all the terms in (7.4.2) and (7.4.4) are locally free sheaves by [Ks, Proposition 1.11.3] since $R^{k}\left(\left.f\right|_{X^{*}}\right)_{*} \iota_{!} \mathbb{Q}_{X^{*} \backslash D^{*}}$ underlies an admissible graded polarized variation of $\mathbb{Q}$-mixed Hodge structure on $Y^{*}$ for every $k$ by Theorem 7.1 (1). See also Proposition 3.12. Let us see the relationship between (7.4.2) and (7.4.4) in details for the reader's convenience. By definition, it is easy to see that

$$
\left({ }^{l} \mathcal{V}_{Y^{*}}^{k}\right)^{*}={ }^{u}\left(\left(\mathcal{V}_{Y^{*}}^{k}\right)^{*}\right)
$$

for every $k$. We can check that

$$
0 \longrightarrow{ }^{u} F^{p}\left(\left(\mathcal{V}_{Y^{*}}^{k}\right)^{*}\right) \longrightarrow\left({ }^{l} \mathcal{V}_{Y^{*}}^{k}\right)^{*} \longrightarrow\left({ }^{l} F^{1-p}\left(\mathcal{V}_{Y^{*}}^{k}\right)\right)^{*} \longrightarrow 0
$$


is exact for every $p$ and $k$ (cf. Lemma 5.1). Then we have the following big commutative diagram.

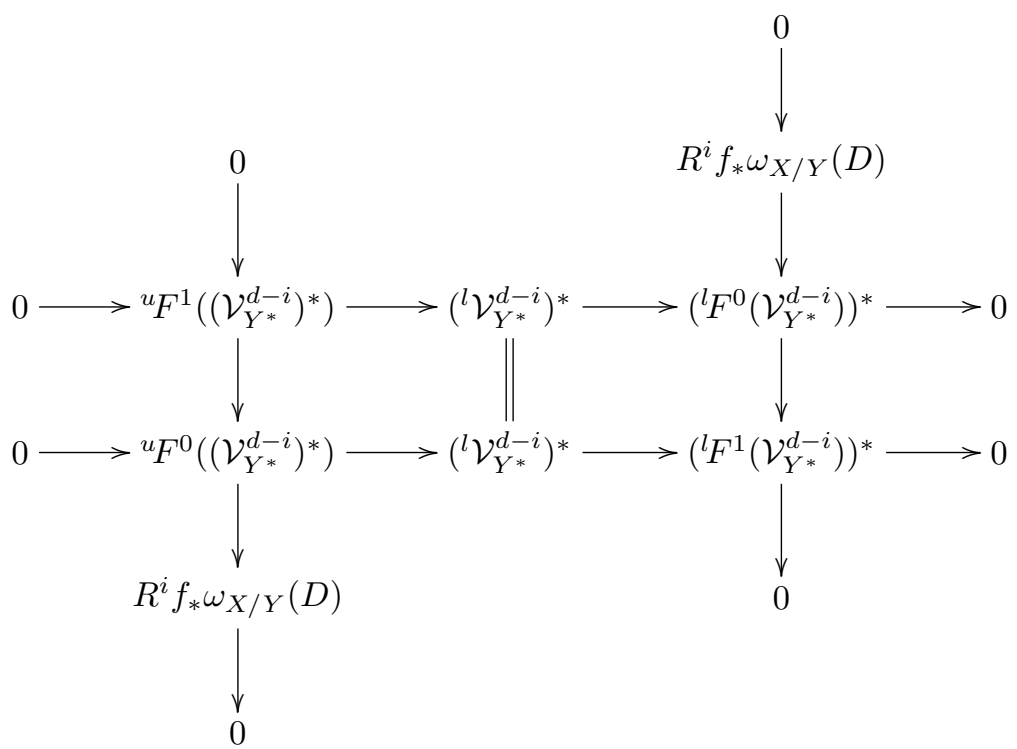

The first vertical line is nothing but (7.4.4) and the third vertical line is the dual of (7.4.2).

Theorem 7.1 (2) (resp. (3)) is a special case of Theorem 7.3 (a) (resp. (b)).

Let us start the proof of Theorem 7.1 and Theorem 7.3.

Proof of Theorem 7.1 and Theorem 7.3. The statement (1) in Theorem 7.1 follows from Theorem 4.15. We note that (4) in Theorem 7.1 follows from Theorem 5.21 and Corollary 5.23 by (3) in Theorem 7.1.

Without loss of generality, by [BiP, Theorem 1.4] and Lemma 6.2, we may assume that $\operatorname{Supp}\left(f^{*} \Sigma \cup D\right)$ is a simple normal crossing divisor on $X$.

In Step 1 and Step 2, we prove (2) and (3) in Theorem 7.1 for every $i$ under the assumption that all the local monodromies on $R^{k} f_{*} \mathbb{C}_{S^{*}}$, where $S$ is a stratum of $(X, D), S^{*}=\left.S\right|_{X^{*}}$, and $k$ is any integer, around $\Sigma$ are unipotent. In Step 3 and Step 4, we prove Theorem 7.3, which contains (2) and (3) in Theorem 7.1.

From now on, we assume that all the local monodromies on $R^{k} f_{*} \mathbb{C}_{S^{*}}$, where $S$ is a stratum of $(X, D), S^{*}=\left.S\right|_{X^{*}}$, and $k$ is any integer, around $\Sigma$ are unipotent.

Step 1 (The case when $\operatorname{dim} Y=1$ ). By shrinking $Y$, we may assume that $Y$ is the unit disc $\Delta$ in $\mathbb{C}$ and $\Sigma=\{0\}$ in $\Delta$. We put $E=f^{-1}(0)$. By considering $\Omega_{(D \cap X) \cdot / Y}\left(\log E_{\bullet}\right)$ as in the proof of Lemma 4.10, we obtain that $R^{d-i} f_{*} \mathcal{O}_{X}(-D)$ 
is isomorphic to the canonical extension of $\operatorname{Gr}_{F}^{0}\left(\mathcal{V}_{Y^{*}}^{d-i}\right)$ for every $i$ (see also Remark 4.16). Therefore, we obtain $R^{i} f_{*} \omega_{X / Y}(D) \simeq\left(\operatorname{Gr}_{F}^{0}\left(\mathcal{V}_{Y}^{d-i}\right)\right)^{*}$ for every $i$ by Grothendieck duality.

Step 2 (The case when $l:=\operatorname{dim} Y \geq 2$ ). We shall prove the statement (3) by induction on $l$ for every $i$.

By Step 1, there is an open subset $Y_{1}$ of $Y$ such that $\operatorname{codim}\left(Y \backslash Y_{1}\right) \geq 2$ and that

$$
\left.\left.R^{i} f_{*} \omega_{X / Y}(D)\right|_{Y_{1}} \simeq\left(\operatorname{Gr}_{F}^{0}\left(\mathcal{V}_{Y}^{d-i}\right)\right)^{*}\right|_{Y_{1}} .
$$

Since $\left(\operatorname{Gr}_{F}^{0}\left(\mathcal{V}_{Y}^{d-i}\right)\right)^{*}$ is locally free (see Remark 7.4), we obtain a homomorphism

$$
\varphi_{Y}^{i}: R^{i} f_{*} \omega_{X / Y}(D) \longrightarrow\left(\operatorname{Gr}_{F}^{0}\left(\mathcal{V}_{Y}^{d-i}\right)\right)^{*}
$$

We will prove that $\varphi_{Y}^{i}$ is an isomorphism. Without loss of generality, we may assume that $X$ and $Y$ are quasi-projective by shrinking $Y$. By Corollary 6.6, $R^{i} f_{*} \omega_{X / Y}(D)$ is torsion-free. Therefore, $\operatorname{Ker} \varphi_{Y}^{i}=0$. We put $G_{Y}^{i}:=\operatorname{Coker} \varphi_{Y}^{i}$. Taking a general hyperplane cut, we see that $\operatorname{Supp} G_{Y}^{i}$ is a finite set by the induction hypothesis. Assume that $G_{Y}^{i} \neq 0$. We may also assume that $\operatorname{Supp} G_{Y}^{i}=\{P\}$ by shrinking $Y$. Let $\mu: W \longrightarrow Y$ be the blowing up at $P$ and set $E=\mu^{-1}(P)$. Then $E \simeq \mathbb{P}^{l-1}$. By [BiM, Theorem 1.5] and [BiP, Theorem 1.4], we can take a projective birational morphism $\pi: X^{\prime} \longrightarrow X$ from a simple normal crossing variety $X^{\prime}$ with the following properties:

(i) the composition $X^{\prime} \longrightarrow X \longrightarrow Y \rightarrow W$ is a morphism.

(ii) $\pi$ is an isomorphism over $X^{*}$.

(iii) $\operatorname{Exc}(\pi) \cup D^{\prime}$ is a simple normal crossing divisor on $X^{\prime}$, where $D^{\prime}$ is the strict transform of $D$.

We obtain that $R^{q} f_{*} \omega_{X / Y}(D) \simeq R^{q}(f \circ \pi)_{*} \omega_{X^{\prime} / Y}\left(D^{\prime}\right)$ for every $q$ because $R \pi_{*} \omega_{X^{\prime}}\left(D^{\prime}\right) \simeq$ $\omega_{X}(D)$ in the derived category of coherent sheaves on $X$ by Lemma 6.2. We note that every stratum of $\left(X^{\prime}, D^{\prime}\right)$ is dominant onto $Y$. We also note the following commutative diagram.

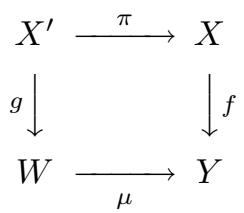

By replacing $(X, D)$ with $\left(X^{\prime}, D^{\prime}\right)$, we may assume that there is a morphism $g$ : $X \longrightarrow W$ such that $f=\mu \circ g$. Since $g: X \longrightarrow W$ is in the same situation as $f$, we obtain the exact sequence:

$$
0 \longrightarrow R^{i} g_{*} \omega_{X / W}(D) \longrightarrow\left(\operatorname{Gr}_{F}^{0}\left(\mathcal{V}_{W}^{d-i}\right)\right)^{*} \longrightarrow G_{W}^{i} \longrightarrow 0 .
$$


Tensoring $\mathcal{O}_{W}(\nu E)$ for $0 \leq \nu \leq l-1$ and applying $R^{j} \mu_{*}$ for $j \geq 0$ to each $\nu$, we have a exact sequence

$$
\begin{aligned}
& 0 \longrightarrow \mu_{*}\left(R^{i} g_{*} \omega_{X / W}(D) \otimes \mathcal{O}_{W}(\nu E)\right) \longrightarrow \mu_{*}\left(\left(\operatorname{Gr}_{F}^{0}\left(\mathcal{V}_{W}^{d-i}\right)\right)^{*} \otimes \mathcal{O}_{W}(\nu E)\right) \\
& \longrightarrow \mu_{*}\left(G_{W}^{i} \otimes \mathcal{O}_{W}(\nu E)\right) \longrightarrow R^{1} \mu_{*}\left(R^{i} g_{*} \omega_{X / W}(D) \otimes \mathcal{O}_{W}(\nu E)\right) \\
& \longrightarrow R^{1} \mu_{*}\left(\left(\operatorname{Gr}_{F}^{0}\left(\mathcal{V}_{W}^{d-i}\right)\right)^{*} \otimes \mathcal{O}_{W}(\nu E)\right) \longrightarrow 0
\end{aligned}
$$

and $R^{q} \mu_{*}\left(R^{i} g_{*} \omega_{X / W}(D) \otimes \mathcal{O}_{W}(\nu E)\right) \simeq R^{q} \mu_{*}\left(\left(\operatorname{Gr}_{F}^{0}\left(\mathcal{V}_{W}^{d-i}\right)\right)^{*} \otimes \mathcal{O}_{W}(\nu E)\right)$ for $q \geq 2$.

By [Kw2, Proposition 1] and Remark 7.4, we obtain

$$
\left(\operatorname{Gr}_{F}^{0}\left(\mathcal{V}_{W}^{d-i}\right)\right)^{*} \simeq \mu^{*}\left(\operatorname{Gr}_{F}^{0}\left(\mathcal{V}_{Y}^{d-i}\right)\right)^{*}
$$

We have

$$
\mu_{*}\left(\left(\operatorname{Gr}_{F}^{0}\left(\mathcal{V}_{W}^{d-i}\right)\right)^{*} \otimes \mathcal{O}_{W}(\nu E)\right) \simeq\left(\operatorname{Gr}_{F}^{0}\left(\mathcal{V}_{Y}^{d-i}\right)\right)^{*}
$$

and

$$
R^{q} \mu_{*}\left(\left(\operatorname{Gr}_{F}^{0}\left(\mathcal{V}_{W}^{d-i}\right)\right)^{*} \otimes \mathcal{O}_{W}(\nu E)\right)=0
$$

for $q \geq 1$. Therefore, $R^{q} \mu_{*}\left(R^{i} g_{*} \omega_{X / W}(D) \otimes \mathcal{O}_{W}(\nu E)\right)=0$ for $q \geq 2$ and

$$
\begin{aligned}
0 \longrightarrow \mu_{*}\left(R^{i} g_{*} \omega_{X / W}(D) \otimes \mathcal{O}_{W}(\nu E)\right) \longrightarrow \mu_{*}\left(\left(\operatorname{Gr}_{F}^{0}\left(\mathcal{V}_{W}^{d-i}\right)\right)^{*} \otimes \mathcal{O}_{W}(\nu E)\right) \\
\quad \longrightarrow \mu_{*}\left(G_{W}^{i} \otimes \mathcal{O}_{W}(\nu E)\right) \longrightarrow R^{1} \mu_{*}\left(R^{i} g_{*} \omega_{X / W}(D) \otimes \mathcal{O}_{W}(\nu E)\right) \\
\quad \longrightarrow 0
\end{aligned}
$$

is exact. Since $\omega_{W}=\mu^{*} \omega_{Y} \otimes \mathcal{O}_{W}((l-1) E)$, we have a spectral sequence

$$
E_{2}^{p, q}=R^{p} \mu_{*}\left(R^{q} g_{*} \omega_{X / W}(D) \otimes \mathcal{O}_{W}((l-1) E)\right) \Longrightarrow R^{p+q} f_{*} \omega_{X / Y}(D) .
$$

However, $E_{2}^{p, q}=0$ for $p \geq 2$ by the above argument. Thus

$$
\begin{aligned}
0 \longrightarrow R^{1} \mu_{*} R^{i-1} g_{*} \omega_{X / Y}(D) \longrightarrow R^{i} f_{*} \omega_{X / Y}(D) \\
\longrightarrow \mu_{*}\left(R^{i} g_{*} \omega_{X / W}(D) \otimes \mathcal{O}_{W}((l-1) E)\right) \longrightarrow 0
\end{aligned}
$$

By Corollary $6.6, R^{i} f_{*} \omega_{X / Y}(D)$ is torsion-free. So, we obtain

$$
R^{1} \mu_{*} R^{i-1} g_{*} \omega_{X / Y}(D)=0 .
$$

Therefore, for $q \geq 1$, we obtain
(A) $R^{i} f_{*} \omega_{X / Y}(D) \simeq \mu_{*}\left(R^{i} g_{*} \omega_{X / W}(D) \otimes \mathcal{O}_{W}((l-1) E)\right)$ and
(B) $R^{q} \mu_{*}\left(R^{i} g_{*} \omega_{X / W}(D) \otimes \mathcal{O}_{W}((l-1) E)\right)=0$

for every $i$. 
Next, we shall consider the following commutative diagram.

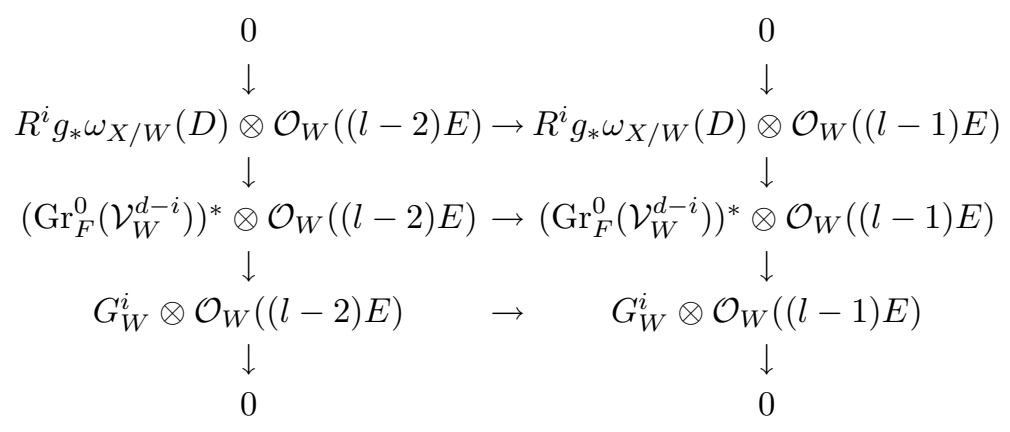

By applying $\mu_{*}$, we have the next commutative diagram.

$$
\begin{aligned}
& \begin{array}{ll}
0 & 0
\end{array} \\
& \downarrow \quad \downarrow \\
& \mu_{*}\left(R^{i} g_{*} \omega_{X / W}(D) \otimes \mathcal{O}_{W}((l-2) E)\right) \rightarrow \mu_{*}\left(R^{i} g_{*} \omega_{X / W}(D) \otimes \mathcal{O}_{W}((l-1) E)\right) \\
& \downarrow \quad \downarrow \\
& \left(\operatorname{Gr}_{F}^{0}\left(\mathcal{V}_{Y}^{d-i}\right)\right)^{*} \quad \simeq \quad \quad\left(\operatorname{Gr}_{F}^{0}\left(\mathcal{V}_{Y}^{d-i}\right)\right)^{*} \\
& \mu_{*}\left(G_{W}^{i} \otimes \mathcal{O}_{W}((l-2) E)\right) \quad \rightarrow \quad \mu_{*}\left(G_{W}^{i} \otimes \mathcal{O}_{W}((l-1) E)\right) \\
& 0
\end{aligned}
$$

By $(\mathrm{A})$ and $(\mathrm{B}), G_{Y}^{i} \simeq \mu_{*}\left(G_{W}^{i} \otimes \mathcal{O}_{W}((l-1) E)\right)$ and

$$
\mu_{*}\left(G_{W}^{i} \otimes \mathcal{O}_{W}((l-2) E)\right) \longrightarrow \mu_{*}\left(G_{W}^{i} \otimes \mathcal{O}_{W}((l-1) E)\right)
$$

is surjective. Since $\operatorname{dim} \operatorname{Supp} G_{W}^{i}=0$ and $E \cap \operatorname{Supp} G_{W}^{i} \neq \emptyset$, it follows that $G_{W}^{i}=0$ by Nakayama's lemma. Therefore, $G_{Y}^{i}=0$. This implies $R^{i} f_{*} \omega_{X / Y}(D) \simeq$ $\left(\operatorname{Gr}_{F}^{0}\left(\mathcal{V}_{Y}^{d-i}\right)\right)^{*}$. By Grothendieck duality, $R^{d-i} f_{*} \mathcal{O}_{X}(-D) \simeq \operatorname{Gr}_{F}^{0}\left(\mathcal{V}_{Y}^{d-i}\right)$.

From now on, we treat the general case, that is, we do not assume that local monodromies are unipotent.

Step 3. In this step, we prove the local freeness of $R^{i} f_{*} \omega_{X / Y}(D)$ for every $i$. We use the unipotent reduction with respect to all the local systems after shrinking $Y$ suitably. This means that, shrinking $Y$, we have the following commutative diagram:

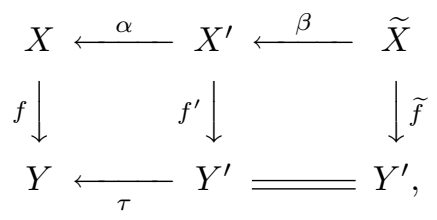


which satisfies the following properties.

(i) $\tau: Y^{\prime} \longrightarrow Y$ is a finite Kummer covering from a nonsingular variety $Y^{\prime}$ and $\tau$ ramifies only along $\Sigma$.

(ii) $f^{\prime}: X^{\prime} \longrightarrow Y^{\prime}$ is the base change of $f: X \longrightarrow Y$ by $\tau$ over $Y \backslash \Sigma$.

(iii) $\left(X^{\prime}, \alpha^{*} D\right)$ is a semi divisorial log terminal pair in the sense of Kollár (see Definition 2.4). Let $X_{j}$ be any irreducible component of $X$. Then $X_{j}^{\prime}=\alpha^{-1}\left(X_{j}\right)$ is the normalization of the base change of $X_{j} \longrightarrow Y$ by $\tau: Y^{\prime} \longrightarrow Y$ and $X^{\prime}=\bigcup_{j} X_{j}^{\prime}$. We note that $X_{j}^{\prime}$ is a $V$-manifold for every $j$. More precisely, $X_{j}^{\prime}$ is toroidal for every $j$.

(iv) $\beta$ is a projective birational morphism from a simple normal crossing variety $\widetilde{X}$ and $\widetilde{D} \cup \operatorname{Exc}(\beta)$ is a simple normal crossing divisor on $\widetilde{X}$, where $\widetilde{D}$ is the strict transform of $\alpha^{*} D$ (cf. [BiM, Theorem 1.5] and [BiP, Theorem 1.4]). We may further assume that $\beta$ is an isomorphism over the largest Zariski open set $U$ of $X^{\prime}$ such that $\left.\left(X^{\prime}, \alpha^{*} D\right)\right|_{U}$ is a simple normal crossing pair.

(v) $\widetilde{f}: \widetilde{X} \longrightarrow Y^{\prime}, \widetilde{D}$, and $\tau^{-1} \Sigma$ satisfy the conditions and assumptions in Theorem 7.1 and all the local monodromies on all the local systems around $\tau^{-1} \Sigma$ are unipotent.

Therefore, $R^{i} \widetilde{f}_{*} \omega_{\widetilde{X}}(\widetilde{D})$ is locally free by Step 1 and Step 2 . On the other hand, we can prove

$$
R^{p} \widetilde{f}_{*} \omega_{\widetilde{X}}(\widetilde{D}) \simeq R^{p} f_{*}^{\prime} \omega_{X^{\prime}}\left(\alpha^{*} D\right)
$$

for every $p \geq 0$. We note that

$$
K_{\widetilde{X}}+\widetilde{D}=\beta^{*}\left(K_{X^{\prime}}+\alpha^{*} D\right)+F
$$

where $F$ is $\beta$-exceptional, $F$ is permissible on $\tilde{X}, \operatorname{Supp} F$ is a simple normal crossing divisor on $\widetilde{X}$, and $\lceil F\rceil$ is effective. Thus we obtain that $\beta_{*} \omega_{\widetilde{X}}(\widetilde{D}) \simeq \omega_{X^{\prime}}\left(\alpha^{*} D\right)$ and that $R^{q} \beta_{*} \omega_{\widetilde{X}}(\widetilde{D})=0$ for every $q>0$ by Lemma 6.1 . Thus, $R^{i} f_{*}^{\prime} \omega_{X^{\prime}}\left(\alpha^{*} D\right)$ is locally free for every $i$. Since $R^{i} f_{*} \omega_{X}(D)$ is a direct summand of

$$
\tau_{*} R^{i} f_{*}^{\prime} \omega_{X^{\prime}}\left(\alpha^{*} D\right) \simeq R^{i} f_{*}\left(\alpha_{*} \omega_{X^{\prime}}\left(\alpha^{*} D\right)\right),
$$

we obtain that $R^{i} f_{*} \omega_{X}(D)$ is locally free, equivalently, $R^{i} f_{*} \omega_{X / Y}(D)$ is locally free for every $i$. We note that, by Grothendieck duality, $R^{d-i} f_{*} \mathcal{O}_{X}(-D)$ is also locally free for every $i$.

Step 4. In this last step, we prove that $R^{d-i} f_{*} \mathcal{O}_{X}(-D)$ is the lower canonical extension for every $i$. By Grothendieck duality and Step 3, $R^{d-i} \widetilde{f}_{*} \mathcal{O}_{\widetilde{X}}(-\widetilde{D})$ is locally free. By Step 3 , we obtain $R \beta_{*} \omega_{\widetilde{X}}(\widetilde{D}) \simeq \omega_{X^{\prime}}\left(\alpha^{*} D\right)$ in the derived category 
of coherent sheaves on $X^{\prime}$. Therefore, we obtain

$$
\begin{aligned}
R \beta_{*} \mathcal{O}_{\widetilde{X}}(-\widetilde{D}) & \simeq R \mathcal{H o m}\left(R \beta_{*} \omega_{\widetilde{X}}^{\bullet}(\widetilde{D}), \omega_{X^{\prime}}^{\bullet}\right) \\
& \simeq R \mathcal{H o m}\left(\omega_{X^{\prime}}^{\bullet}\left(\alpha^{*} D\right), \omega_{X^{\prime}}^{\bullet}\right) \simeq \mathcal{O}_{X^{\prime}}\left(-\alpha^{*} D\right)
\end{aligned}
$$

in the derived category of coherent sheaves on $X^{\prime}$. Note that $X^{\prime}$ is Cohen-Macaulay (cf. $\left[\right.$ F14, Theorem 4.2]) and that $\omega_{X^{\prime}}^{\bullet} \simeq \omega_{X^{\prime}}\left[\operatorname{dim} X^{\prime}\right]$. Thus, we have

$$
R^{p} \widetilde{f}_{*} \mathcal{O}_{\widetilde{X}}(-\widetilde{D}) \simeq R^{p} f_{*}^{\prime} \mathcal{O}_{X^{\prime}}\left(-\alpha^{*} D\right)
$$

for every $p$. Let $G$ be the Galois group of $\tau: Y^{\prime} \longrightarrow Y$. Then we have

$$
\left(\tau_{*} R^{p} f_{*}^{\prime} \mathcal{O}_{X^{\prime}}\left(-\alpha^{*} D\right)\right)^{G} \simeq R^{p} f_{*}\left(\alpha_{*} \mathcal{O}_{X^{\prime}}\left(-\alpha^{*} D\right)\right)^{G} \simeq R^{p} f_{*} \mathcal{O}_{X}(-D) .
$$

Thus, we obtain that $R^{d-i} f_{*} \mathcal{O}_{X}(-D)$ is the lower canonical extension for every $i$ (cf. [Ko2, Notation 2.5 (iii)]). By Grothendieck duality, $R^{i} f_{*} \omega_{X / Y}(D)$ is the upper canonical extension for every $i$.

We finish the proof of Theorem 7.1 and Theorem 7.3.

The following theorem is a generalization of [Ko1, Proposition 7.6].

Theorem 7.5. Let $f: X \longrightarrow Y$ be a projective surjective morphism from a simple normal crossing variety to a smooth algebraic variety $Y$ with connected fibers. Assume that every stratum of $X$ is dominant onto $Y$. Then $R^{d} f_{*} \omega_{X} \simeq \omega_{Y}$ where $d=\operatorname{dim} X-\operatorname{dim} Y$.

Proof. By [BiM, Theorem 1.5] and [BiP, Theorem 1.4], we can construct a commutative diagram

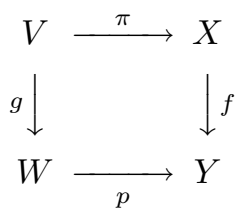

with the following properties.

(i) $p: W \longrightarrow Y$ is a projective birational morphism from a smooth quasiprojective variety $W$.

(ii) $V$ is a simple normal crossing variety.

(iii) $\pi$ is projective birational and $\pi$ induces an isomorphism $\pi^{0}=\left.\pi\right|_{V^{0}}: V^{0} \longrightarrow$ $X^{0}$ where $X^{0}$ (resp. $V^{0}$ ) is a Zariski open set of $X$ (resp. $V$ ) which contains the generic point of any stratum of $X$ (resp. $V$ ).

(iv) $g$ is projective. 
(v) there is a simple normal crossing divisor $\Sigma$ on $W$ such that every stratum of $V$ is smooth over $W \backslash \Sigma$.

We note that $R^{j} g_{*} \omega_{V}$ is locally free for every $j$ by Theorem 7.3. By Grothendieck duality, we have

$$
R g_{*} \mathcal{O}_{V} \simeq R \mathcal{H} \operatorname{om}_{\mathcal{O}_{W}}\left(R g_{*} \omega_{V}^{\bullet}, \omega_{W}^{\bullet}\right)
$$

Therefore, we have

$$
\mathcal{O}_{W} \simeq \mathcal{H o m}_{\mathcal{O}_{W}}\left(R^{d} g_{*} \omega_{V}, \omega_{W}\right)
$$

Note that, by Zariski's main theorem, $f_{*} \mathcal{O}_{X} \simeq \mathcal{O}_{Y}$ since every stratum of $X$ is dominant onto $Y$. Therefore, $g_{*} \mathcal{O}_{V} \simeq \mathcal{O}_{W}$. Thus, we obtain $R^{d} g_{*} \omega_{V} \simeq \omega_{W}$. By applying $p_{*}$, we have $p_{*} R^{d} g_{*} \omega_{V} \simeq p_{*} \omega_{W} \simeq \omega_{Y}$. We note that $p_{*} R^{d} g_{*} \omega_{V} \simeq$ $R^{d}(p \circ g)_{*} \omega_{V}$ since $R^{i} p_{*} R^{d} g_{*} \omega_{V}=0$ for every $i>0$ (cf. Theorem 6.3 (ii)). On the other hand,

$$
R^{d}(p \circ g)_{*} \omega_{V} \simeq R^{d}(f \circ \pi)_{*} \omega_{V} \simeq R^{d} f_{*} \omega_{X}
$$

since $R^{i} \pi_{*} \omega_{V}=0$ for every $i>0$ by Lemma 6.1 and $\pi_{*} \omega_{V} \simeq \omega_{X}$ (cf. Lemma 6.2). Therefore, we obtain $R^{d} f_{*} \omega_{X} \simeq \omega_{Y}$.

In geometric applications, we sometimes have a projective surjective morphism $f: X \longrightarrow Y$ from a simple normal crossing variety to a smooth variety $Y$ with connected fibers such that every stratum of $X$ is mapped onto $Y$. The example below shows that in general there is no stratum $S$ of $X$ such that general fibers of $S \longrightarrow Y$ are connected. Therefore, Kawamata's result ([Kw3, Theorem 1.1]) is very restrictive. He assumes that $S \longrightarrow Y$ has connected fibers for every stratum $S$ of $X$.

Example 7.6. We consider $W=\mathbb{P}^{1} \times \mathbb{P}^{1} \times \mathbb{P}^{1}$. Let $p_{i}: \mathbb{P}^{1} \times \mathbb{P}^{1} \times \mathbb{P}^{1} \longrightarrow \mathbb{P}^{1}$ be the $i$-th projection for $i=1,2,3$. We take general members $X_{1} \in\left|p_{1}^{*} \mathcal{O}_{\mathbb{P}^{1}}(1) \otimes p_{2}^{*} \mathcal{O}_{\mathbb{P}^{1}}(2)\right|$ and $X_{2} \in\left|p_{1}^{*} \mathcal{O}_{\mathbb{P}^{1}}(1) \otimes p_{3}^{*} \mathcal{O}_{\mathbb{P}^{1}}(2)\right|$. We define $X=X_{1} \cup X_{2}, Y=\mathbb{P}^{1}$, and $f=\left.p_{1}\right|_{X}$ : $X \longrightarrow Y$. Then $f$ is a projective morphism from a simple normal crossing variety $X$ to a smooth projective curve $Y$. We can directly check that

$$
H^{1}\left(W, \mathcal{O}_{W}\left(-X_{1}\right)\right)=H^{1}\left(W, \mathcal{O}_{W}\left(-X_{2}\right)\right)=0
$$

and

$$
H^{1}\left(W, \mathcal{O}_{W}\left(-X_{1}-X_{2}\right)\right)=H^{2}\left(W, \mathcal{O}_{W}\left(-X_{1}-X_{2}\right)\right)=0 .
$$

Therefore, by using

$$
0 \longrightarrow \mathcal{O}_{W}\left(-X_{1}-X_{2}\right) \longrightarrow \mathcal{O}_{W}\left(-X_{2}\right) \longrightarrow \mathcal{O}_{X_{1}}\left(-X_{2}\right) \longrightarrow 0,
$$

we obtain $H^{1}\left(X_{1}, \mathcal{O}_{X_{1}}\left(-X_{2}\right)\right)=0$. By using

$$
0 \longrightarrow \mathcal{O}_{X_{1}}\left(-X_{2}\right) \longrightarrow \mathcal{O}_{X_{1}} \longrightarrow \mathcal{O}_{X_{1} \cap X_{2}} \longrightarrow 0,
$$


we obtain $H^{0}\left(X_{1} \cap X_{2}, \mathcal{O}_{X_{1} \cap X_{2}}\right)=\mathbb{C}$ since $H^{0}\left(X_{1}, \mathcal{O}_{X_{1}}\right)=\mathbb{C}$. This means that $C=X_{1} \cap X_{2}$ is a smooth connected curve. Therefore, every stratum of $X$ is mapped onto $Y$ by $f$. We note that general fibers of $f: X_{1} \longrightarrow Y, f: X_{2} \longrightarrow Y$, and $f: C \longrightarrow Y$ are disconnected.

As a special case of Theorem 7.1, we obtain the following theorem.

Theorem 7.7 (cf. [Kw1, Theorem 5], [Ko2, Theorem 2.6], and [N1, Theorem 1]). Let $f: X \longrightarrow Y$ be a projective morphism between smooth complete algebraic varieties which satisfies the following conditions:

(i) There is a Zariski open subset $Y^{*}$ of $Y$ such that $\Sigma=Y \backslash Y^{*}$ is a simple normal crossing divisor on $Y$.

(ii) We put $X^{*}=f^{-1}\left(Y^{*}\right)$. Then $\left.f\right|_{X^{*}}$ is smooth.

(iii) The local monodromies of $R^{d+i}\left(\left.f\right|_{X^{*}}\right)_{*} \mathbb{C}_{X^{*}}$ around $\Sigma$ are unipotent, where $d=\operatorname{dim} X-\operatorname{dim} Y$.

Then $R^{i} f_{*} \omega_{X / Y}$ is a semipositive locally free sheaf on $Y$.

Proof. By Poincaré-Verdier duality (see, for example, [PS, Theorem 13.9]), the local system $R^{d-i}\left(\left.f\right|_{X^{*}}\right)_{*} \mathbb{C}_{X^{*}}$ is the dual local system of $R^{d+i}\left(\left.f\right|_{X^{*}}\right)_{*} \mathbb{C}_{X^{*}}$. Therefore, the local monodromies of $R^{d-i}\left(\left.f\right|_{X^{*}}\right)_{*} \mathbb{C}_{X^{*}}$ around $\Sigma$ are unipotent. Thus, by Theorem 7.1, we obtain that $R^{i} f_{*} \omega_{X / Y} \simeq\left(R^{d-i} f_{*} \mathcal{O}_{X}\right)^{*}$ is a semipositive locally free sheaf on $Y$.

Similarly, the semipositivity theorem in [F4] (cf. [F4, Theorem 3.9]) can be recovered by Theorem 7.1 . We note that [Kw3, Theorem 1.1] does not cover [F4, Theorem 3.9]. This is because Kawamata's theorem needs that $S \longrightarrow Y$ has connected fibers for every stratum $S$ of $(X, D)$ (cf. Example 7.6).

Remark 7.8. Let $f: X \longrightarrow Y$ be a projective morphism between smooth projective varieties. Assume that there exists a simple normal crossing divisor $\Sigma$ on $Y$ such that $f$ is smooth over $Y \backslash \Sigma$. Then $R^{i} f_{*} \omega_{X / Y}$ is locally free for every $i$ (cf. Theorem 7.3 and [Ko2, Theorem 2.6]). We note that $R^{i} f_{*} \omega_{X / Y}$ is not always semipositive if we assume nothing on monodromies around $\Sigma$.

We close this section with an easy example.

Example 7.9 (Double cover). We consider $\pi: Y=\mathbb{P}_{\mathbb{P}^{1}}\left(\mathcal{O}_{\mathbb{P}^{1}} \oplus \mathcal{O}_{\mathbb{P}^{1}}(2)\right) \longrightarrow \mathbb{P}^{1}$. Let $E$ and $G$ be the sections of $\pi$ such that $E^{2}=-2$ and $G^{2}=2$. We note that $E+2 F \sim G$ where $F$ is a fiber of $\pi$. We put $\mathcal{L}=\mathcal{O}_{Y}(E+F)$. Then $E+G \in\left|\mathcal{L}^{\otimes 2}\right|$. Let $f: X \longrightarrow Y$ be the double cover constructed by $E+G \in\left|\mathcal{L}^{\otimes 2}\right|$. Then 
$f: X \longrightarrow Y$ is étale outside $\Sigma=E+G$ and

$$
f_{*} \omega_{X / Y} \simeq \mathcal{O}_{Y} \oplus \mathcal{L} .
$$

In this case, $f_{*} \omega_{X / Y}$ is not semipositive since $\mathcal{L} \cdot E=-1$. We note that the local monodromies on $\left(\left.f\right|_{X^{*}}\right)_{*} \mathbb{C}_{X^{*}}$ around $\Sigma$ are not unipotent, where $Y^{*}=Y \backslash \Sigma$ and $X^{*}=f^{-1}\left(Y^{*}\right)$.

In Example 7.9, $f: X \longrightarrow Y$ is finite and the general fibers of $f$ are disconnected. In Section 8, we discuss an example $f: X \longrightarrow Y$ whose general fibers are elliptic curves such that $f_{*} \omega_{X / Y}$ is not semipositive (cf. Corollary 8.10 and Example 8.16).

\section{§8. Examples}

In this final section, we give supplementary examples for the Fujita-Kawamata semipositivity theorem (cf. [Kw1, Theorem 5]), Viehweg's weak positivity theorem, and the Fujino-Mori canonical bundle formula (cf. $[\mathrm{FM}]$ ). For details of the original Fujita-Kawamata semipositivity theorem, see, for example, [Mor, §5] and [F5, Section 5].

8.1 (Semipositivity in the sense of Fujita-Kawamata). The following example is due to Takeshi Abe. It is a small remark on Definition 5.20.

Example 8.2. Let $C$ be an elliptic curve and let $E$ be a stable vector bundle on $C$ such that the degree of $E$ is -1 and the rank of $E$ is two. Let $f_{m}: C \longrightarrow C$ be the multiplication by $m$ where $m$ is a positive integer. In this case, every quotient line bundle $L$ of $E$ has non-negative degree. However, $\mathcal{O}_{\mathbb{P}(E)}(1)$ is not nef. It is because we can find a quotient line bundle $M$ of $f_{m}^{*} E$ whose degree is negative for some positive integer $m$.

8.3 (Canonical bundle formula). We give sample computations of our canonical bundle formula obtained in $[\mathrm{FM}]$. We will freely use the notation in $[\mathrm{FM}]$. For details of our canonical bundle formula, see [FM], [F2, §3], and [F3, $33, \S 4, \S 5$, and $\S 6]$.

8.4 (Kummer manifolds). Let $E$ be an elliptic curve and let $E^{n}$ be the $n$-times direct product of $E$. Let $G$ be the cyclic group of order two of analytic automorphisms of $E^{n}$ generated by an automorphism

$$
\tau: E^{n} \longrightarrow E^{n}:\left(z_{1}, \cdots, z_{n}\right) \mapsto\left(-z_{1}, \cdots,-z_{n}\right) .
$$

The automorphism $\tau$ has $2^{2 n}$ fixed points. Each singular point is terminal for $n \geq 3$ and is canonical for $n \geq 2$. 
8.5 (Kummer surfaces). First, we consider $q: E^{2} / G \longrightarrow E / G \simeq \mathbb{P}^{1}$, which is induced by the first projection, and $g=q \circ \mu: Y \longrightarrow \mathbb{P}^{1}$, where $\mu: Y \longrightarrow E^{2} / G$ is the minimal resolution of sixteen $A_{1}$-singularities. It is easy to see that $Y$ is a $K 3$ surface. In this case, it is obvious that

$$
g_{*} \mathcal{O}_{Y}\left(m K_{Y / \mathbb{P}^{1}}\right) \simeq \mathcal{O}_{\mathbb{P}^{1}}(2 m)
$$

for every $m \geq 1$. Thus, we can put $L_{Y / \mathbb{P}^{1}}=D$ for any degree two Weil divisor $D$ on $\mathbb{P}^{1}$. For the definition of $L_{Y / \mathbb{P}^{1}}$, see [FM, Definition 2.3]. We obtain $K_{Y}=$ $g^{*}\left(K_{\mathbb{P}^{1}}+L_{Y / \mathbb{P}^{1}}\right)$. Let $Q_{i}$ be the branch point of $E \longrightarrow E / G \simeq \mathbb{P}^{1}$ for $1 \leq i \leq 4$. Then we have

$$
L_{Y / \mathbb{P}^{1}}^{s s}=D-\sum_{i=1}^{4}\left(1-\frac{1}{2}\right) Q_{i}=D-\sum_{i=1}^{4} \frac{1}{2} Q_{i}
$$

by the definition of the semi-stable part $L_{Y / \mathbb{P}^{1}}^{s s}$ (see [FM, Proposition 2.8, Definition 4.3, and Proposition 4.7]). Therefore, we obtain

$$
K_{Y}=g^{*}\left(K_{\mathbb{P}^{1}}+L_{Y / \mathbb{P}^{1}}^{s s}+\sum_{i=1}^{4} \frac{1}{2} Q_{i}\right) .
$$

Thus,

$$
L_{Y / \mathbb{P}^{1}}^{s s}=D-\sum_{i=1}^{4} \frac{1}{2} Q_{i} \nsim 0
$$

but

$$
2 L_{Y / \mathbb{P}^{1}}^{s s}=2 D-\sum_{i=1}^{4} Q_{i} \sim 0 .
$$

Note that $L_{Y / \mathbb{P}^{1}}^{s s}$ is not a Weil divisor but a $\mathbb{Q}$-Weil divisor on $\mathbb{P}^{1}$.

8.6 (Elliptic fibrations). Next, we consider $E^{3} / G$ and $E^{2} / G$. We consider the morphism $p: E^{3} / G \longrightarrow E^{2} / G$ induced by the projection $E^{3} \longrightarrow E^{2}:\left(z_{1}, z_{2}, z_{3}\right) \mapsto$ $\left(z_{1}, z_{2}\right)$. Let $\nu: X^{\prime} \longrightarrow E^{3} / G$ be the weighted blow-up of $E^{3} / G$ at sixty-four $\frac{1}{2}(1,1,1)$-singularities. Thus

$$
K_{X^{\prime}}=\nu^{*} K_{E^{3} / G}+\sum_{j=1}^{64} \frac{1}{2} E_{j}
$$

where $E_{j} \simeq \mathbb{P}^{2}$ is the exceptional divisor for every $j$. Let $P_{i}$ be an $A_{1}$-singularity of $E^{2} / G$ for $1 \leq i \leq 16$. Let $\psi: X \longrightarrow X^{\prime}$ be the blow-up of $X^{\prime}$ along the strict transform of $p^{-1}\left(P_{i}\right)$, which is isomorphic to $\mathbb{P}^{1}$, for every $i$. Then we obtain the 
following commutative diagram.

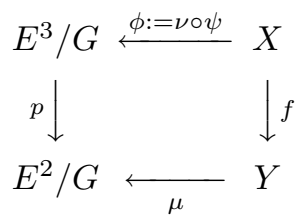

Note that

$$
K_{X}=\phi^{*} K_{E^{3} / G}+\sum_{j=1}^{64} \frac{1}{2} E_{j}+\sum_{k=1}^{16} F_{k},
$$

where $E_{j}$ is the strict transform of $E_{j}$ on $X$ and $F_{k}$ is the $\psi$-exceptional prime divisor for every $k$. We can check that $X$ is a smooth projective threefold. We put $C_{i}=\mu^{-1}\left(P_{i}\right)$ for every $i$. It can be checked that $C_{i}$ is a $(-2)$-curve for every $i$. It is easily checked that $f$ is smooth outside $\sum_{i=1}^{16} C_{i}$ and that the degeneration of $f$ is of type $I_{0}^{*}$ along $C_{i}$ for every $i$. We renumber $\left\{E_{j}\right\}_{j=1}^{64}$ as $\left\{E_{i}^{j}\right\}$, where $f\left(E_{i}^{j}\right)=C_{i}$ for every $1 \leq i \leq 16$ and $1 \leq j \leq 4$. We note that $f$ is flat since $f$ is equidimensional.

Let us recall the following theorem (cf. [Kw2, Theorem 20] and [N2, Corollary 3.2.1 and Theorem 3.2.3]).

Theorem 8.7 (..., Kawamata, Nakayama, ...). We have the following isomorphism.

$$
\left(f_{*} \omega_{X / Y}\right)^{\otimes 12} \simeq \mathcal{O}_{Y}\left(\sum_{i=1}^{16} 6 C_{i}\right)
$$

where $\omega_{X / Y} \simeq \mathcal{O}_{X}\left(K_{X / Y}\right)=\mathcal{O}_{X}\left(K_{X}-f^{*} K_{Y}\right)$.

The proof of Theorem 8.7 depends on the investigation of the upper canonical extension of the Hodge filtration and the period map. It is obvious that

$$
2 K_{X}=f^{*}\left(2 K_{Y}+\sum_{i=1}^{16} C_{i}\right)
$$

and

$$
2 m K_{X}=f^{*}\left(2 m K_{Y}+m \sum_{i=1}^{16} C_{i}\right)
$$

for all $m \geq 1$ since $f^{*} C_{i}=2 F_{i}+\sum_{j=1}^{4} E_{i}^{j}$ for every $i$. Therefore, we have $2 L_{X / Y} \sim$ $\sum_{i=1}^{16} C_{i}$. On the other hand, $f_{*} \omega_{X / Y} \simeq \mathcal{O}_{Y}\left(\left\lfloor L_{X / Y}\right\rfloor\right)$. Note that $Y$ is a smooth surface and $f$ is flat. Since

$$
\mathcal{O}_{Y}\left(12\left\lfloor L_{X / Y}\right\rfloor\right) \simeq\left(f_{*} \omega_{X / Y}\right)^{\otimes 12} \simeq \mathcal{O}_{Y}\left(\sum_{i=1}^{16} 6 C_{i}\right),
$$


we have

$$
12 L_{X / Y} \sim 6 \sum_{i=1}^{16} C_{i} \sim 12\left\lfloor L_{X / Y}\right\rfloor .
$$

Thus, $L_{X / Y}$ is a Weil divisor on $Y$. It is because the fractional part $\left\{L_{X / Y}\right\}$ is effective and linearly equivalent to zero. So, $L_{X / Y}$ is numerically equivalent to $\frac{1}{2} \sum_{i=1}^{16} C_{i}$. We have $g^{*} Q_{i}=2 G_{i}+\sum_{j=1}^{4} C_{i}^{j}$ for every $i$. Here, we renumbered $\left\{C_{j}\right\}_{j=1}^{16}$ as $\left\{C_{i}^{j}\right\}_{i, j=1}^{4}$ such that $g\left(C_{i}^{j}\right)=Q_{i}$ for every $i$ and $j$. More precisely, we put $2 G_{i}=g^{*} Q_{i}-\sum_{j=1}^{4} C_{i}^{j}$ for every $i$. We note that we used notations in 8.5. We consider $A:=g^{*} D-\sum_{i=1}^{4} G_{i}$. Then $A$ is a Weil divisor and $2 A \sim \sum_{i=1}^{16} C_{i}$. Thus, $A$ is numerically equivalent to $\frac{1}{2} \sum_{i=1}^{16} C_{i}$. Since $H^{1}\left(Y, \mathcal{O}_{Y}\right)=0$, we can put $L_{X / Y}=A$. So, we have

$$
L_{X / Y}^{s s}=g^{*} D-\sum_{i=1}^{4} G_{i}-\sum_{j=1}^{16} \frac{1}{2} C_{j} .
$$

We obtain the following canonical bundle formula.

Theorem 8.8. The next formula holds.

$$
K_{X}=f^{*}\left(K_{Y}+L_{X / Y}^{s s}+\sum_{j=1}^{16} \frac{1}{2} C_{j}\right)
$$

where $L_{X / Y}^{s s}=g^{*} D-\sum_{i=1}^{4} G_{i}-\sum_{j=1}^{16} \frac{1}{2} C_{j}$.

We note that $2 L_{X / Y}^{s s} \sim 0$ but $L_{X / Y}^{s s} \nsim 0$. The semi-stable part $L_{X / Y}^{s s}$ is not a Weil divisor but a $\mathbb{Q}$-divisor on $Y$.

The next lemma is obvious since the index of $K_{E^{3} / G}$ is two. We give a direct proof here.

Lemma 8.9. $H^{0}\left(Y, L_{X / Y}\right)=0$.

Proof. Suppose that there exists an effective Weil divisor $B$ on $Y$ such that $L_{X / Y} \sim$ $B$. Since $B \cdot C_{i}=-1$, we have $B \geq \frac{1}{2} C_{i}$ for all $i$. Thus $B \geq \sum_{i=1}^{16} \frac{1}{2} C_{i}$. This implies that $B-\sum_{i=1}^{16} \frac{1}{2} C_{i}$ is an effective $\mathbb{Q}$-divisor and is numerically equivalent to zero. Thus $B=\sum_{i=1}^{16} \frac{1}{2} C_{i}$. It is a contradiction.

We can easily check the following corollary.

Corollary 8.10. We have

$$
f_{*} \omega_{X / Y}^{\otimes m} \simeq \begin{cases}\mathcal{O}_{Y}\left(\sum_{i=1}^{16} n C_{i}\right) & \text { if } m=2 n \\ \mathcal{O}_{Y}\left(L_{X / Y}+\sum_{i=1}^{16} n C_{i}\right) & \text { if } m=2 n+1 .\end{cases}
$$


In particular, $f_{*} \omega_{X / Y}^{\otimes m}$ is not nef for any $m \geq 1$. We can also check that

$$
H^{0}\left(Y, f_{*} \omega_{X / Y}^{\otimes m}\right) \simeq \begin{cases}\mathbb{C} & \text { if } m \text { is even }, \\ 0 & \text { if } m \text { is odd } .\end{cases}
$$

Corollary 8.10 shows that [T, Theorem 1.9 (1)] is sharp.

$\mathbf{8 . 1 1}$ (Weak positivity). Let us recall the definition of Viehweg's weak positivity (cf. [V1, Definition 1.2] and [V3, Definition 2.11]). The reader can find some interesting applications of a generalization of Viehweg's weak positivity theorem in [FG1].

Definition 8.12 (Weak positivity). Let $W$ be a smooth quasi-projective variety and let $\mathcal{F}$ be a locally free sheaf on $W$. Let $U$ be an open subvariety of $W$. Then, $\mathcal{F}$ is weakly positive over $U$ if for every ample invertible sheaf $\mathcal{H}$ and every positive integer $\alpha$ there exists some positive integer $\beta$ such that $S^{\alpha \cdot \beta}(\mathcal{F}) \otimes \mathcal{H}^{\beta}$ is generated by global sections over $U$ where $S^{k}$ denotes the $k$-th symmetric product for every positive integer $k$. This means that the natural map

$$
H^{0}\left(W, S^{\alpha \cdot \beta}(\mathcal{F}) \otimes \mathcal{H}^{\beta}\right) \otimes \mathcal{O}_{W} \longrightarrow S^{\alpha \cdot \beta}(\mathcal{F}) \otimes \mathcal{H}^{\beta}
$$

is surjective over $U$.

Remark 8.13 (cf. [V1, (1.3) Remark. iii)]). In Definition 8.12, it is enough to check the condition for one invertible sheaf $\mathcal{H}$, not necessarily ample, and all $\alpha>0$. For details, see [V3, Lemma 2.14 a)].

Remark 8.14. In [V2, Definition 3.1], $S^{\alpha \cdot \beta}(\mathcal{F}) \otimes \mathcal{H}^{\otimes \beta}$ is only required to be generically generated. See also [Mor, (5.1) Definition].

We explicitly check the weak positivity for the elliptic fibration constructed in 8.6 (cf. [V1, Theorem 4.1 and Theorem III] and [V3, Theorem 2.41 and Corollary $2.45])$.

Proposition 8.15. Let $m$ be a positive integer. Let $f: X \longrightarrow Y$ be the elliptic fibration constructed in 8.6. Then $f_{*} \omega_{X / Y}^{\otimes m}$ is weakly positive over $Y_{0}=Y \backslash \sum_{i=1}^{16} C_{i}$. Let $U$ be a Zariski open set such that $U \not \subset Y_{0}$. Then $f_{*} \omega_{X / Y}^{\otimes m}$ is not weakly positive over $U$.

Proof. Let $H$ be a very ample Cartier divisor on $Y$ such that $L_{X / Y}+H$ is very ample. We put $\mathcal{H}=\mathcal{O}_{Y}(H)$. Let $\alpha$ be an arbitrary positive integer. Then

$$
S^{\alpha}\left(f_{*} \omega_{X / Y}^{\otimes m}\right) \otimes \mathcal{H} \simeq \mathcal{O}_{Y}\left(\alpha \sum_{i=1}^{16} n C_{i}+H\right)
$$


if $m=2 n$. When $m=2 n+1$, we have

$$
\begin{aligned}
& S^{\alpha}\left(f_{*} \omega_{X / Y}^{\otimes m}\right) \otimes \mathcal{H} \\
& \simeq\left\{\begin{array}{l}
\mathcal{O}_{Y}\left(\alpha \sum_{i=1}^{16} n C_{i}+H+L_{X / Y}+\left\lfloor\frac{\alpha}{2}\right\rfloor \sum_{i=1}^{16} C_{i}\right) \text { if } \alpha \text { is odd, } \\
\mathcal{O}_{Y}\left(\alpha \sum_{i=1}^{16} n C_{i}+H+\frac{\alpha}{2} \sum_{i=1}^{16} C_{i}\right) \text { if } \alpha \text { is even. }
\end{array}\right.
\end{aligned}
$$

Thus, $S^{\alpha}\left(f_{*} \omega_{X / Y}^{\otimes m}\right) \otimes \mathcal{H}$ is generated by global sections over $Y_{0}$ for every $\alpha>0$. Therefore, $f_{*} \omega_{X / Y}^{\otimes m}$ is weakly positive over $Y_{0}$.

Let $\mathcal{A}$ be an ample invertible sheaf on $Y$. We put $k=\max _{j}\left(C_{j} \cdot \mathcal{A}\right)$. Let $\alpha$ be a positive integer with $\alpha>k / 2$. We note that

$$
S^{2 \alpha \cdot \beta}\left(f_{*} \omega_{X / Y}^{\otimes m}\right) \otimes \mathcal{A}^{\otimes \beta} \simeq\left(\mathcal{O}_{Y}\left(\alpha \sum_{i=1}^{16} m C_{i}\right) \otimes \mathcal{A}\right)^{\otimes \beta} .
$$

If $H^{0}\left(Y, S^{2 \alpha \cdot \beta}\left(f_{*} \omega_{X / Y}^{\otimes m}\right) \otimes \mathcal{A}^{\otimes \beta}\right) \neq 0$, then we can take

$$
G \in\left|\left(\mathcal{O}_{Y}\left(\alpha \sum_{i=1}^{16} m C_{i}\right) \otimes \mathcal{A}\right)^{\otimes \beta}\right|
$$

In this case, $G \cdot C_{i}<0$ for every $i$ because $\alpha>k / 2$. Therefore, we obtain $G \geq$ $\sum_{i=1}^{16} C_{i}$. Thus, $S^{2 \alpha \cdot \beta}\left(f_{*} \omega_{X / Y}^{\otimes m}\right) \otimes \mathcal{A}^{\otimes \beta}$ is not generated by global sections over $U$ for any $\beta \geq 1$. This means that $f_{*} \omega_{X / Y}^{\otimes m}$ is not weakly positive over $U$.

Proposition 8.15 implies that [V3, Corollary 2.45] is the best result.

Example 8.16. Let $f: X \longrightarrow Y$ be the elliptic fibration constructed in 8.6. Let $Z:=C \times X$, where $C$ is a smooth projective curve with the genus $g(C)=r \geq 2$. Let $\pi_{1}: Z \longrightarrow C$ (resp. $\pi_{2}: Z \longrightarrow X$ ) be the first (resp. second) projection. We put $h:=f \circ \pi_{2}: Z \longrightarrow Y$. In this case, $K_{Z}=\pi_{1}^{*} K_{C} \otimes \pi_{2}^{*} K_{X}$. Therefore, we obtain

$$
h_{*} \omega_{Z / Y}^{\otimes m}=f_{*} \pi_{2 *}\left(\pi_{1}^{*} \omega_{C}^{\otimes m} \otimes \pi_{2}^{*} \omega_{X}^{\otimes m}\right) \otimes \omega_{Y}^{\otimes-m}=\left(f_{*} \omega_{X / Y}^{\otimes m}\right)^{\oplus l},
$$

where $l=\operatorname{dim} H^{0}\left(C, \mathcal{O}_{C}\left(m K_{C}\right)\right)$. Thus, $l=(2 m-1) r-2 m+1$ if $m \geq 2$ and $l=r$ if $m=1$. So, $h_{*} \omega_{Z / Y}$ is a rank $r \geq 2$ vector bundle on $Y$ such that $h_{*} \omega_{Z / Y}$ is not semipositive. We note that $h$ is smooth over $Y_{0}=Y \backslash \sum_{i=1}^{16} C_{i}$. We also note that $h_{*} \omega_{Z / Y}^{\otimes m}$ is weakly positive over $Y_{0}$ for every $m \geq 1$ by [V3, Theorem 2.41 and Corollary 2.45].

Example 8.16 shows that the assumption on the local monodromies around $\sum_{i=1}^{16} C_{i}$ is indispensable for the semipositivity theorem.

We close this section with a comment on [FM]. 
8.17 (Comment). We give a remark on [FM, Section 4]. In [FM, 4.4], $g: Y \longrightarrow X$ is a $\log$ resolution of $(X, \Delta)$. However, it is better to assume that $g$ is a log resolution of $\left(X, \Delta-(1 / b) B^{\Delta}\right)$ for the proof of [FM, Theorem 4.8].

\section{Acknowledgements}

The first author was partially supported by The Inamori Foundation and by the Grant-in-Aid for Young Scientists (A) $\sharp 24684002$ from JSPS. He would like to thank Professors Takeshi Abe, Hiraku Kawanoue, Kenji Matsuki, and Shigefumi Mori for discussions. He also thanks Professors Valery Alexeev and Christopher Hacon for introducing him this problem. He thanks Professor Gregory James Pearlstein and Professor Fouad El Zein for answering his questions and giving him some useful comments. He thanks Professor Kazuya Kato for fruitful discussions. The authors would like to thank Professors Kazuya Kato, Chikara Nakayama, Sampei Usui, and Morihiko Saito for discussions and comments.

\section{References}

[Be] B. Berndtsson, Curvature of vector bundles associated to holomorphic fibrations, Ann. of Math. (2) 169 (2009), no. 2, 531-560.

[BeP] B. Berndtsson, M. Păun, Bergman kernels and the pseudoeffectivity of relative canonical bundles, Duke Math. J. 145 (2008), no. 2, 341-378.

[BiM] E. Bierstone, P. D. Milman, Resolution except for minimal singularities I, Adv. Math. 231 (2012), no. 5, 3022-3053.

[BiP] E. Bierstone, F. Vera Pacheco, Resolution of singularities of pairs preserving semi-simple normal crossings, Rev. R. Acad. Cienc. Exactas Fís. Nat. Ser. A Math. RACSAM 107 (2013), no. 1, 159-188.

[Bo] A. Borel et al., Algebraic D-modules, Perspectives in Mathematics, 2. Academic Press, Inc., Boston, MA, 1987.

[BZ] J.-L. Brylinski, S. Zucker, An overview of recent advances in Hodge theory, Several complex variables, VI, 39-142, Encyclopaedia Math. Sci., 69, Springer, Berlin, 1990.

[CK] E. Cattani, A. Kaplan, Polarized mixed Hodge structures and the local monodromy of a variation of Hodge structure, Invent. Math. 67 (1982), no. 1, 101-115.

[CKS] E. Cattani, A. Kaplan, W. Schmid, Degeneration of Hodge structures, Ann. of Math. (2) 123 (1986), no. 3, 457-535.

[D1] P. Deligne, Equations Différentielles à Points Singuliers Réguliers, Lecture Notes in Math., 163, Springer-Verlag, 1970.

[D2] P. Deligne, Théorie de Hodge II, Inst. Hautes Études Sci. Publ. Math. 40 (1971), 5-57.

[D3] P. Deligne, Théorie de Hodge III, Inst. Hautes Études Sci. Publ. Math. 44 (1972), 5-77.

[dB] P. Du Bois, Structure de Hodge mixte sur la cohomologie évanescente, Ann. Inst. Fourier (Grenoble) 35 (1985), no. 1, 191-213.

[E1] F. El Zein, Théorie de Hodge des cycles évanescents, Ann. Sci. École Norm. Sup. (4) 19 (1986), no. 1, 107-184.

[E2] F. El Zein, Introduction á la théorie de Hodge mixte, Actualités Mathématiques, Hermann, Paris, 1991. 
[FGAE] B. Fantechi, L. Göttsch, L. Illusie, S.L. Kleinman, N. Nitsure, A. Vistoli, Fundamental Algebraic Geometry, Mathematical Surveys and Monographs, 123. American Math. Soc., 2005.

[F1] O. Fujino, Abundance theorem for semi log canonical threefolds, Duke Math. J. 102 (2000), no. 3, 513-532.

[F2] O. Fujino, Algebraic fiber spaces whose general fibers are of maximal Albanese dimension, Nagoya Math. J. 172 (2003), 111-127.

[F3] O. Fujino, A canonical bundle formula for certain algebraic fiber spaces and its applications, Nagoya Math. J. 172 (2003), 129-171.

[F4] O. Fujino, Higher direct images of log canonical divisors, J. Differential Geom. 66 (2004), no. 3, 453-479.

[F5] O. Fujino, Remarks on algebraic fiber spaces, J. Math. Kyoto Univ. 45 (2005), no. 4, 683-699.

[F6] O. Fujino, What is log terminal?, in Flips for 3-folds and 4-folds (Alessio Corti, ed.), 49-62, Oxford University Press, 2007.

[F7] O. Fujino, Introduction to the log minimal model program for log canonical pairs, preprint (2009).

[F8] O. Fujino, On injectivity, vanishing and torsion-free theorems for algebraic varieties, Proc. Japan Acad. Ser. A Math. Sci. 85 (2009), no. 8, 95-100.

[F9] O. Fujino, Theory of non-lc ideal sheaves: basic properties, Kyoto Journal of Mathematics, Vol. 50, No. 2 (2010), 225-245.

[F10] O. Fujino, Introduction to the theory of quasi-log varieties, Classification of algebraic varieties, 289-303, EMS Ser. Congr. Rep., Eur. Math. Soc., Zürich, 2011,

[F11] O. Fujino, Fundamental theorems for the log minimal model program, Publ. Res. Inst. Math. Sci. 47 (2011), no. 3, 727-789.

[F12] O. Fujino, Minimal model theory for log surfaces, Publ. Res. Inst. Math. Sci. 48 (2012), no. 2, 339-371.

[F13] O. Fujino, On isolated log canonical singularities with index one, J. Math. Sci. Univ. Tokyo 18 (2011), 299-323.

[F14] O. Fujino, Vanishing theorems, to appear in Adv. Stud. Pure Math.

[F15] O. Fujino, Fundamental theorems for semi log canonical pairs, Algebraic Geometry 1 (2014), no. 2, 194-228.

[F16] O. Fujino, Semipositivity theorems for moduli problems, preprint (2012).

[F17] O. Fujino, Injectivity theorems, to appear in Adv. Stud. Pure Math.

[FFS] O. Fujino, T. Fujisawa, M. Saito, Some remarks on the semipositivity theorems, Publ. Res. Inst. Math. Sci. 50 (2014), no. 1, 85-112.

[FG1] O. Fujino, Y. Gongyo, On images of weak Fano manifolds II, to appear in Algebraic and Complex Geometry - In Honour of Klaus Hulek's 60th Birthday.

[FG2] O. Fujino, Y. Gongyo, On the moduli b-divisors of lc-trivial fibrations, to appear in Ann. Inst. Fourier (Grenoble) 64 (2014).

[FM] O. Fujino, S. Mori, A canonical bundle formula, J. Differential Geom. 56 (2000), no. 1, 167-188.

[Ft] T. Fujita, On Kähler fiber spaces over curves, J. Math. Soc. Japan 30 (1978), no. 4, 779-794.

[G] P. A. Griffiths, Periods of integrals on algebraic manifolds. III. Some global differentialgeometric properties of the period mapping, Inst. Hautes Études Sci. Publ. Math. No. 38 (1970), 125-180. 
[GN] F. Guillén, V. Navarro Aznar, Sur le théorème local des cycles invariants, Duke Math. J. 61 (1990), no. 1, 133-155.

[GNPP] F. Guillén, V. Navarro Aznar, P. Pascual Gainza, F. Puerta, Hyperrésolutions cubiques et descente cohomologique, Lecture Notes in Mathematics, 1335. SpringerVerlag, Berlin, 1988.

[Ks] M. Kashiwara, A study of variation of mixed Hodge structure, Publ. Res. Inst. Math. Sci. 22 (1986), no. 5, 991-1024.

[Kt] N. Katz, An Overview of Deligne's Work on Hilbert's Twenty-First Problem, Proceedings of Symposia in Pure Mathematics 28, (1976), 537-557

[KO] N. Katz, T. Oda, On the differentiation of De Rham cohomology classes with respect to parameters, J. Math. Kyoto Univ. 8 (1968), 199-213.

[Kw1] Y. Kawamata, Characterization of abelian varieties, Compositio Math. 43 (1981), no. $2,253-276$.

[Kw2] Y. Kawamata, Kodaira dimension of certain algebraic fiber spaces, J. Fac. Sci. Univ. Tokyo Sect. IA Math. 30 (1983), no. 1, 1-24.

[Kw3] Y. Kawamata, Semipositivity theorem for reducible algebraic fiber spaces, Pure Appl. Math. Q. 7 (2011), no. 4, Special Issue: In memory of Eckart Viehweg, 1427-1447.

[Ko1] J. Kollár, Higher direct images of dualizing sheaves, I. Ann. of Math. 123 (1986), 11-42.

[Ko2] J. Kollár, Higher direct images of dualizing sheaves, II. Ann. of Math. 124 (1986), 171-202.

[Ko3] J. Kollár, Subadditivity of the Kodaira dimension: fibers of general type, Algebraic geometry, Sendai, 1985, 361-398, Adv. Stud. Pure Math., 10, North-Holland, Amsterdam, 1987.

[Ko4] J. Kollár, Projectivity of complete moduli, J. Differential Geom. 32 (1990), no. 1, 235268.

[Ko5] J. Kollár, Kodaira's canonical bundle formula and adjunction, Flips for 3-folds and 4-folds, 134-162, Oxford Lecture Ser. Math. Appl., 35, Oxford Univ. Press, Oxford, 2007.

[Ko6] J. Kollár, Singularities of the Minimal Model Program, With the collaboration of S. Kovács. Cambridge Tracts in Mathematics, 200. Cambridge University Press, Cambridge, 2013.

[Moc] S. Mochizuki, On semi-positivity and filtered Frobenius crystals, Publ. Res. Inst. Math. Sci. 31 (1995), no. 1, 81-94.

[Mor] S. Mori, Classification of higher-dimensional varieties, Algebraic geometry, Bowdoin, 1985 (Brunswick, Maine, 1985), 269-331, Proc. Sympos. Pure Math., 46, Part 1, Amer. Math. Soc., Providence, RI, 1987.

[MT] C. Mourougane, S. Takayama, Extension of twisted Hodge metrics for Kähler morphisms, J. Differential Geom. 83 (2009), no. 1, 131-161.

[Mu] D. Mumford, Abelian Varieties, Oxford University Press, 1970,

[N1] N. Nakayama, Hodge filtrations and the higher direct images of canonical sheaves, Invent. Math. 85 (1986), no. 1, 217-221.

[N2] N. Nakayama, Local structure of an elliptic fibration, Higher dimensional birational geometry (Kyoto, 1997), 185-295, Adv. Stud. Pure Math., 35, Math. Soc. Japan, Tokyo, 2002.

[NA] V. Navarro Aznar, Sur la théorie de Hodge-Deligne, Invent. Math. 90 (1987), no. 1, 11-76.

[PS] C. Peters, J. Steenbrink, Mixed Hodge structures, Ergebnisse der Mathematik und ihrer Grenzgebiete. 3. Folge. A Series of Modern Surveys in Mathematics, 52. Springer-Verlag, 
Berlin, 2008.

[Sa] M. Saito, Modules de Hodge polarisables, Publ. Res. Inst. Math. Sci. 24 (1988), no. 6, 849-995.

[Sc] W. Schmid, Variation of Hodge structure: the singularities of the period mapping, Invent. Math. 22 (1973), 211-319.

[SSU] M.-H. Saito, Y. Shimizu, S. Usui, Variation of mixed Hodge structure and the Torelli problem, Algebraic geometry, Sendai, 1985, 649-693, Adv. Stud. Pure Math., 10, North-Holland, Amsterdam, 1987.

[St1] J. Steenbrink, Limits of Hodge structures, Invent. Math. 31 (1975/76), no. 3, 229-257.

[St2] J. Steenbrink, Mixed Hodge structure on the vanishing cohomology, Real and complex singularities (Proc. Ninth Nordic Summer School/NAVF Sympos. Math., Oslo, 1976), pp. 525-563. Sijthoff and Noordhoff, Alphen aan den Rijn, 1977.

[SZ] J. Steenbrink, S. Zucker, Variation of mixed Hodge structure, I. Invent. Math. 80 (1985), no. 3, 489-542.

[T] H. Tsuji, Global generation of the direct images of relative pluricanonical systems, preprint (2010).

[U] S. Usui, Mixed Torelli problem for Todorov surfaces, Osaka J. Math. 28 (1991) 697-735

[V1] E. Viehweg, Weak positivity and the additivity of the Kodaira dimension for certain fibre spaces, Algebraic varieties and analytic varieties (Tokyo, 1981), 329-353, Adv. Stud. Pure Math., 1, North-Holland, Amsterdam, 1983.

[V2] E. Viehweg, Weak positivity and the additivity of the Kodaira dimension. II. The local Torelli map, Classification of algebraic and analytic manifolds (Katata, 1982), 567-589, Progr. Math., 39, Birkhäuser Boston, Boston, MA, 1983.

[V3] E. Viehweg, Quasi-projective moduli for polarized manifolds, Ergebnisse der Mathematik und ihrer Grenzgebiete (3), 30. Springer-Verlag, Berlin, 1995.

[Z] S. Zucker, Remarks on a theorem of Fujita, J. Math. Soc. Japan, 34 (1982), 47-54. 